\title{
WHITTAKER PERIODS, MOTIVIC PERIODS, AND SPECIAL VALUES OF TENSOR PRODUCT $L$-FUNCTIONS
}

\author{
HARALD GROBNER ${ }^{1}$ AND MICHAEL HARRIS ${ }^{2,3,4,5}$ \\ ${ }^{1}$ Fakultät für Mathematik, Universität Wien, Oskar-Morgenstern-Platz 1, \\ A-1090 Wien, Austria (harald.grobner@univie.ac.at) \\ ${ }^{2}$ Univ Paris Diderot, Sorbonne Paris Cité, UMR 7586, Institut de Mathématiques \\ de Jussieu-Paris Rive Gauche, Case 247, 4 place Jussieu F-75005, Paris, France \\ ${ }^{3}$ Sorbonne Universités, UPMC Univ Paris 06, UMR 7586, IMJ-PRG, \\ F-75005 Paris, France \\ ${ }^{4}$ CNRS, UMR7586, IMJ-PRG, F-75013 Paris, France \\ ${ }^{5}$ Department of Mathematics, Columbia University, New York, NY 10027, USA
}

(Received 28 August 2014; revised 27 December 2014; accepted 28 December 2014; first published online 31 March 2015)

\begin{abstract}
Let $\mathcal{K}$ be an imaginary quadratic field. Let $\Pi$ and $\Pi^{\prime}$ be irreducible generic cohomological automorphic representation of $\mathrm{GL}(n) / \mathcal{K}$ and $\mathrm{GL}(n-1) / \mathcal{K}$, respectively. Each of them can be given two natural rational structures over number fields. One is defined by the rational structure on topological cohomology, and the other is given in terms of the Whittaker model. The ratio between these rational structures is called a Whittaker period. An argument presented by Mahnkopf and Raghuram shows that, at least if $\Pi$ is cuspidal and the weights of $\Pi$ and $\Pi^{\prime}$ are in a standard relative position, the critical values of the Rankin-Selberg product $L\left(s, \Pi \times \Pi^{\prime}\right)$ are essentially algebraic multiples of the product of the Whittaker periods of $\Pi$ and $\Pi^{\prime}$. We show that, under certain regularity and polarization hypotheses, the Whittaker period of a cuspidal $\Pi$ can be given a motivic interpretation, and can also be related to a critical value of the adjoint $L$-function of related automorphic representations of unitary groups. The resulting expressions for critical values of the Rankin-Selberg and adjoint $L$-functions are compatible with Deligne's conjecture.
\end{abstract}

Keywords: cuspidal automorphic representation; Deligne's conjecture; special values; $L$-function; periods; rationality

2010 Mathematics subject classification: Primary 11F67

Secondary 11F41; 11F70; 11F75; 22E55

H.G. is supported in part by the Austrian Science Fund (FWF) Erwin Schrödinger grant, J 3076-N13, and the FWF-project, project number P 25974-N25. The research leading to these results has received funding from the European Research Council under the European Community's Seventh Framework Programme (FP7/2007-2013)/ERC Grant agreement no. 290766 (AAMOT). 


\section{Contents}

$\begin{array}{lll}1 & \text { Introduction } & 713\end{array}$

2 Unitary groups and base change $\quad \mathbf{7 1 8}$

2.1 Some standing assumptions and notation . . . . . . . . . . . 718

2.2 Base change . . . . . . . . . . . . . . . . . . . 720

2.3 Descent to unitary groups and base change . . . . . . . . . . . . 720

2.4 The cuspidal automorphic representation $\Pi \ldots \ldots$. . . . . . . . . . . 721

2.5 The abelian automorphic representation $\Pi^{\prime} \ldots \ldots \ldots$. . . . . . . . 721

2.6 An action of Aut(C) . . . . . . . . . . . . . . . . . . 722

2.7 Rational structures on cohomological Harish-Chandra modules . . . . . . 724

3 Whittaker periods for the general linear group $\quad \mathbf{7 2 5}$

3.1 Automorphic cohomology of locally symmetric spaces . . . . . . . . . 725

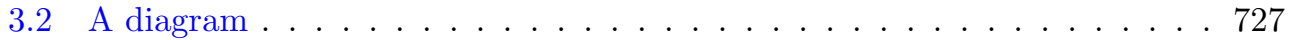

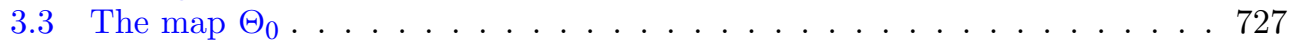

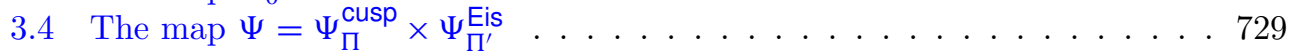

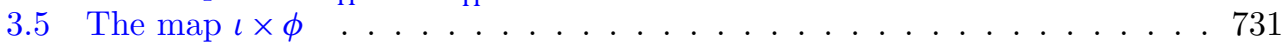

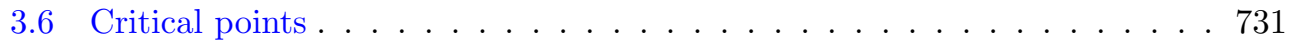

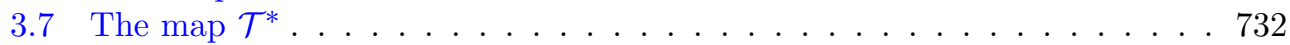

3.8 Poincaré duality . . . . . . . . . . . . . . . . . . . . . . . . . . . . . . . . . . . . . . . . . . . . . . . . . . . .

3.9 A non-archimedean, particular vector . . . . . . . . . . . . 733

3.10 An archimedean non-vanishing result . . . . . . . . . . . . . . . . 734

3.11 A theorem on Whittaker periods . . . . . . . . . . . . 735

4 Motives - an interlude $\quad \mathbf{7 3 9}$

4.1 Tensor products of motives . . . . . . . . . . . . . . . . . . . . . . . . . . . . . . . . .

4.2 Critical values when $n^{\prime}=n-1 \ldots \ldots \ldots$. . . . . . . . . . . . . . . . . . . . . . . 741

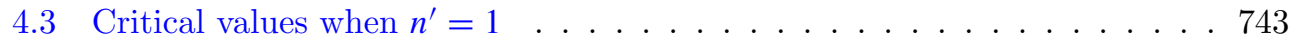

4.4 Holomorphic automorphic forms on unitary groups and automorphic critical intervals when $n^{\prime}=1 \ldots \ldots$. . . . . . . . . . . 744

4.5 A comparison to Deligne's periods . . . . . . . . . . . . . . . . 749

4.6 The case of a Hecke character . . . . . . . . . . . . . . . . 750

$\begin{array}{lll}5 & \text { Rational Eisenstein classes of abelian type } & \mathbf{7 5 1}\end{array}$

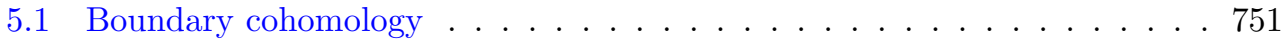

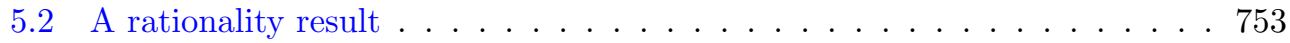

5.3 Whittaker coefficients . . . . . . . . . . . . . 754

6 Period relations - the main results $\quad \mathbf{7 5 5}$

6.1 Critical values of tensor products when one of the representations is of

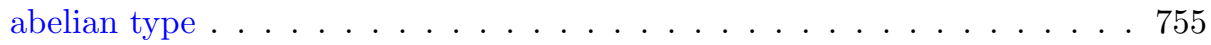

6.2 Whittaker periods and Petersson norms . . . . . . . . . . . . . 756

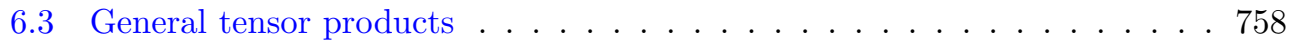

6.4 The archimedean constant . . . . . . . . . . . . . . . . . 759

6.5 Whittaker periods and adjoint $L$-values . . . . . . . . . . . . . . . 759

6.6 Generalizations . . . . . . . . . . . . . . . . . . 764

$\begin{array}{ll}\text { References } & \mathbf{7 6 6}\end{array}$ 


\section{Introduction}

$L$-functions can be attached both to automorphic representations and to arithmetic objects such as Galois representations or motives, and one implication of the Langlands program is that $L$-functions of the second kind are examples of $L$-functions of the first kind. Very few results of arithmetic interest can be proved about the second kind of $L$-functions until they have been identified with automorphic $L$-functions. For example, there is an extraordinarily deep web of conjectures relating the values at integer points of arithmetic (motivic) $L$-functions to cohomological invariants of the corresponding geometric (motivic) objects. In practically all the instances ${ }^{1}$ where these conjectures have been proved, automorphic methods have proved indispensable.

At the same time, there is a growing number of results on special values of automorphic $L$-functions that make no direct reference to arithmetic. Instead, the special values are written as algebraic multiples of complex invariants defined by means of representation theory. Examples relevant to the present paper include [16, 34, 38, 39], where the complex invariants are defined for representations of cohomological type by reference to the uniqueness of Whittaker or Shalika models. This paper continues this series, proving a version of the main results of $[34,38,39]$ for the Rankin-Selberg $L$-function of a pair $\left(\Pi, \Pi^{\prime}\right)$ of a cuspidal automorphic representation $\Pi$ of $\mathrm{GL}_{n}$ of cohomological type, and a cuspidal or abelian automorphic representation $\Pi^{\prime}$ of $\mathrm{GL}_{n-1}$, also of cohomological type, where the general linear groups are over an imaginary quadratic field $\mathcal{K}$. Here, the notion 'abelian automorphic' refers to a representation which is a tempered Eisenstein representation induced from a Borel subgroup of $\mathrm{GL}_{n-1}$. In view of Raghuram's recent preprint [38], which we received after writing a first version of this paper, the inclusion of such automorphic representations $\Pi^{\prime}$ is the new feature of this result.

Our cohomological result is stated below as Theorem 1.3. The main purpose of this paper is to bring this purely automorphic result closer to the motivic expression for the critical values of the Rankin-Selberg $L$-function $L\left(s, \Pi \times \Pi^{\prime}\right)$ embodied in Deligne's conjecture [8]. In what follows, we let $c$ denote complex conjugation of $\mathcal{K}$. Assume for the moment that both $\Pi$ and $\Pi^{\prime}$ are cuspidal. In order to identify $L\left(s, \Pi \times \Pi^{\prime}\right)$ with a motivic $L$-function, we make the following hypothesis (see Hypothesis 4.19 for details).

Hypotheses 1.1. Both $\Pi$ and $\Pi^{\prime}$ can be obtained by base change from holomorphic discrete series representations of unitary groups of arbitrary signature.

Up to twisting by an integral or half-integral power of the global character $g \mapsto$ $\|\operatorname{det}(g)\|$, we may assume that $\Pi$ and $\Pi^{\prime}$ have unitary central character (so the central critical value of $L\left(s, \Pi \times \Pi^{\prime}\right)$ is at $\left.s=\frac{1}{2}\right)$. Then Hypothesis 1.1 implies the polarization condition

$$
\Pi^{\vee} \stackrel{\sim}{\rightarrow} \Pi^{c} \quad \text { and } \quad\left(\Pi^{\prime}\right)^{\vee} \stackrel{\sim}{\rightarrow}\left(\Pi^{\prime}\right)^{c}
$$

Conversely, it has been known for some time ([31]; see also [30]) that the polarization condition implies that, for any Hermitian space $W$ over $\mathcal{K}$ of dimension $n, \Pi$ descends

${ }^{1}$ Whether or not the analytic formulas for special values of the Riemann zeta function and Dirichlet $L$-series should be considered exceptions is beyond the scope of this introduction. 
to a stable $L$-packet $\{\pi\}_{W}$ of cuspidal automorphic representations of $G=U(W)$, of discrete series type at infinity, provided that it descends locally at all finite places; a similar condition holds for $\Pi^{\prime}$. The signature in Hypothesis 1.1 is the invariant of $W$ at a fixed complex embedding of $\mathcal{K}$. In particular, Hypothesis 1.1 is automatic for $\Pi$ (respectively, $\Pi^{\prime}$ ) if $n$ is odd (respectively, even).

Under Hypothesis 1.1, the $L$-packet $\left\{\pi^{\vee}\right\}_{W}$ contains (at least) one member for which $\pi_{\infty}^{\vee}$ belongs to the holomorphic discrete series. Let the signature of $W$ be $(j, n-j)$, and choose an arithmetically normalized vector $\phi_{j}$ in the chosen $\pi^{\vee}$. Define $P^{(j)}(\Pi)$ to be the square norm of $\phi_{j}$ for the (arithmetically normalized) Petersson inner product. The $P^{(j)}(\Pi)$ are the motivic invariants in terms of which we express the critical values of $L\left(s, \Pi \times \Pi^{\prime}\right)$.

More precisely, the arithmetic normalization is defined with reference to the coherent cohomology of the Shimura variety attached to $G$, or rather to the similitude group $\mathrm{GU}(W)$. It thus depends on a choice of extension of $\phi_{j}$ to a (holomorphic) modular form on the similitude group. We ignore this irritating but crucial detail in the introduction; it is addressed at length in Remark 4.33.

Here is our main theorem.

Theorem 1.2. Let $\Pi$ and $\Pi^{\prime}$ be cuspidal automorphic representations of $\mathrm{GL}_{n}\left(\mathbb{A}_{\mathcal{K}}\right)$ and $\mathrm{GL}_{n-1}\left(\mathbb{A}_{\mathcal{K}}\right)$ which are cohomological with respect to irreducible algebraic representations $E_{\mu}$ and $E_{\lambda}$, respectively, where $\mu$ (respectively, $\lambda$ ) is the corresponding highest weight (i.e., an $n$-tuple (respectively, $n-1$-tuple) of integers), assumed to satisfy the equivalent conditions of Lemma 2.1 (i.e., Hypothesis 2.3 for $\mathrm{m}=0$ ). We assume that $\Pi$ and $\Pi^{\prime}$ satisfy Hypothesis 1.1. Let $\mathbb{Q}\left(\Pi_{f}\right)$ and $\mathbb{Q}\left(\Pi_{f}^{\prime}\right)$ be the fields of definition of the finite parts $\Pi_{f}$ and $\Pi_{f}^{\prime}$ of $\Pi$ and $\Pi^{\prime}$, respectively. Furthermore, we assume that the parameters of $\Pi$ and $\Pi^{\prime}$ are sufficiently regular:

$$
\mu_{i}-\mu_{i+1} \geqslant 2 \text { for all } i \text { and } \lambda_{j}-\lambda_{j+1} \geqslant 2 \text { for all } j \text {. }
$$

Then there are finite extensions $E(\Pi) / \mathbb{Q}\left(\Pi_{f}\right)$ and $E\left(\Pi^{\prime}\right) / \mathbb{Q}\left(\Pi_{f}^{\prime}\right)$ such that, for every critical point $s_{0}=\frac{1}{2}+m$ of $L\left(s, \Pi \times \Pi^{\prime}\right)$ with $m \geqslant 0$, there is a non-zero constant $u\left(m, \Pi_{\infty}, \Pi_{\infty}^{\prime}\right)$, depending only on $m, \mu, \lambda$ (or equivalently $\left.m, \Pi_{\infty}, \Pi_{\infty}^{\prime}\right)$, such that

$$
L\left(\frac{1}{2}+m, \Pi_{f} \times \Pi_{f}^{\prime}\right) \sim_{\mathcal{K} E(\Pi) E\left(\Pi^{\prime}\right)} u\left(m, \Pi_{\infty}, \Pi_{\infty}^{\prime}\right) \prod_{j=1}^{n-1} P^{(j)}(\Pi) \prod_{k=1}^{n-2} P^{(k)}\left(\Pi^{\prime}\right),
$$

where $\sim \mathcal{K} E(\Pi) E\left(\Pi^{\prime}\right)$ means up to multiplication by an element in the composition of number fields $\mathcal{K} E(\Pi) E\left(\Pi^{\prime}\right)$. Equivalently, for every critical point $s_{0}=n-1+m$ of $L\left(s, R\left(M(\Pi) \otimes M\left(\Pi^{\prime}\right)\right)\right)$ with $m \geqslant 0$,

$$
\begin{aligned}
& L\left(n-1+m, R\left(M(\Pi) \otimes M\left(\Pi^{\prime}\right)\right)\right) \\
& \quad \sim_{\mathcal{K} E(\Pi) E\left(\Pi^{\prime}\right)} u\left(m, \Pi_{\infty}, \Pi_{\infty}^{\prime}\right) \prod_{j=1}^{n-1} P^{(j)}(\Pi) \prod_{k=1}^{n-2} P^{(k)}\left(\Pi^{\prime}\right) .
\end{aligned}
$$


The fields $\mathbb{Q}\left(\Pi_{f}\right)$ and $\mathbb{Q}\left(\Pi_{f}^{\prime}\right)$ are number fields by [5, Theorem 3.13]. The role of the finite extensions $E(\Pi)$ and $E\left(\Pi^{\prime}\right)$, which can be taken to be cyclotomic fields, is explained below.

The proof involves three main ingredients. The first is the extension mentioned above of the results of Mahnkopf and Raghuram. In what follows, $\Pi$ is cuspidal but $\Pi^{\prime}$ is either cuspidal or abelian automorphic. Since $\Pi$ and $\Pi^{\prime}$ are cohomological, their finite parts $\Pi_{f}$ and $\Pi_{f}^{\prime}$ have natural rational structures over the number fields $\mathbb{Q}\left(\Pi_{f}\right)$ and $\mathbb{Q}\left(\Pi_{f}^{\prime}\right)$, respectively, defined by the rational structure on cohomology with coefficients in the local systems attached to $E_{\mu}$ and $E_{\lambda}$. But since they are tempered, they are both globally generic, and therefore $\Pi_{f}$ and $\Pi_{f}^{\prime}$ have rational structures defined in terms of their Whittaker models. The two rational structures are necessarily proportional, because the representations are irreducible. The factors of proportionality, called Whittaker periods, belong to $\mathbb{C}^{\times}$. Details will be provided in subsequent sections. In what follows, $p(\Pi)$ and $p\left(\Pi^{\prime}\right)$ are the Whittaker periods. Here is a statement.

Theorem 1.3. Let $\Pi=B C(\pi)\|\cdot\|^{\mathrm{m}}$ be a cuspidal automorphic representation of $\mathrm{GL}_{n}\left(\mathbb{A}_{\mathcal{K}}\right), \mathrm{m} \in \mathbb{Z}$, and let $\Pi^{\prime}=B C\left(\pi^{\prime}\right)$ be a cuspidal or abelian automorphic representation of $\mathrm{GL}_{n-1}\left(\mathbb{A}_{\mathcal{K}}\right)$ obtained by base change from unitary groups as in $\S \S 2.4$ and 2.5. In particular, $\Pi$ is cohomological with respect to $E_{\mu}$, and $\Pi^{\prime}$ is cohomological with respect to $E_{\lambda}$. We assume that the parameters $\mu$ and $\lambda$ satisfy Hypothesis 2.3. Then for every critical point $s_{0}=\frac{1}{2}+m$ of $L\left(s, \Pi \times \Pi^{\prime}\right)$ with $m \geqslant 0$, there is a non-zero constant $p\left(m, \Pi_{\infty}, \Pi_{\infty}^{\prime}\right) \in \mathbb{C}^{\times}$, depending only on $m, \mu, \lambda$ (or equivalently $m, \Pi_{\infty}, \Pi_{\infty}^{\prime}$ ), such that the following hold.

(1) For all critical values $\frac{1}{2}+m \in \operatorname{Crit}\left(\Pi \times \Pi^{\prime}\right)$ with $m \geqslant 0$ and every $\sigma \in \operatorname{Aut}(\mathbb{C})$,

$$
\sigma\left(\frac{L\left(\frac{1}{2}+m, \Pi_{f} \times \Pi_{f}^{\prime}\right)}{p(\Pi) p\left(\Pi^{\prime}\right) p\left(m, \Pi_{\infty}, \Pi_{\infty}^{\prime}\right) \mathcal{G}\left(\omega_{\Pi_{f, 0}^{\prime}}\right)}\right)=\frac{L\left(\frac{1}{2}+m,{ }^{\sigma} \Pi_{f} \times{ }^{\sigma} \Pi_{f}^{\prime}\right)}{p\left({ }^{\sigma} \Pi\right) p\left({ }^{\sigma} \Pi^{\prime}\right) p\left(m,{ }^{\sigma} \Pi_{\infty},{ }^{\sigma} \Pi_{\infty}^{\prime}\right) \mathcal{G}\left(\omega_{\sigma} \Pi_{f, 0}^{\prime}\right)} .
$$

$$
L\left(\frac{1}{2}+m, \Pi_{f} \times \Pi_{f}^{\prime}\right) \sim_{\mathbb{Q}\left(\Pi_{f}\right) \mathbb{Q}\left(\Pi_{f}^{\prime}\right)} p(\Pi) p\left(\Pi^{\prime}\right) p\left(m, \Pi_{\infty}, \Pi_{\infty}^{\prime}\right) \mathcal{G}\left(\omega_{\Pi_{f, 0}^{\prime}}\right),
$$

where $\sim_{\mathbb{Q}\left(\Pi_{f}\right) \mathbb{Q}\left(\Pi_{f}^{\prime}\right)}$ means up to multiplication by an element in the composition of number fields $\mathbb{Q}\left(\Pi_{f}\right) \mathbb{Q}\left(\Pi_{f}^{\prime}\right)$.

The proof of this result follows the methods of $[34,38,39]$ : the branching formula for the restriction of the representation $E_{\mu}$ to $\mathrm{GL}_{n-1}$ shows that the Rankin-Selberg integral for $\mathrm{GL}_{n} \times G L_{n-1}$ can be interpreted as a cohomological cup product precisely for the critical values $s_{0}$ of the theorem. On the one hand, the cup product is rational on rational cohomology classes; on the other hand, the Rankin-Selberg integral is, up to an unspecified archimedean factor, a rational multiple of the Euler product on automorphic forms that are rational with respect to the Whittaker rational structure. Comparing these two rationality properties, we obtain the result; the constant $p\left(m, \Pi_{\infty}, \Pi_{\infty}^{\prime}\right)$ accounts for the archimedean Rankin-Selberg integral.

The proof of Theorem 1.3 nevertheless involves two novelties. The first is specific to the method of this paper, and consists in the observation that the method applied 
in the earlier work remains valid when $\Pi^{\prime}$ is abelian automorphic; the reason for this choice will be made apparent below. The second novelty is of more general import. The main contribution to the archimedean invariants $p\left(m, \Pi_{\infty}, \Pi_{\infty}^{\prime}\right)$ is a Rankin-Selberg zeta integral involving explicit cohomological vectors. For nearly 40 years, results of this type, for $\mathrm{GL}_{n}$ with $n>2$, were presented subject to a local non-vanishing hypothesis, which in our present situation comes down to the claim that the archimedean zeta integral does not equal zero for the choice of vectors forced upon us by the method. In 2011, however, Binyong Sun discovered an abstract representation-theoretic method that allowed him to prove that such integrals never vanish. In his companion paper [44] he applies this method to cohomological Rankin-Selberg zeta integrals for $\mathrm{GL}_{n}(\mathbb{R}) \times \mathrm{GL}_{n-1}(\mathbb{R})$ and for $\mathrm{GL}_{n}(\mathbb{C}) \times \mathrm{GL}_{n-1}(\mathbb{C})$. Without Sun's breakthrough, the results of this paper would still be conditioned on the non-vanishing hypothesis.

When $\Pi^{\prime}$ is the abelian automorphic representation attached to an $(n-1)$-tuple of Hecke characters $\chi_{i}$, the $L$-function $L\left(s, \Pi \times \Pi^{\prime}\right)$ factors as a product of $n-1 L$-functions of $\mathrm{GL}_{n}$, twisted by the $\chi_{i}$. When $\Pi^{\prime}$ is cohomological, these $L$-functions are of the type whose special values were considered in [20] (completed by [21]). The second main ingredient of the proof is the expression of these special values in terms of the $P^{(j)}(\Pi)$ introduced in 1.2, and in terms of period invariants attached to the $\chi_{i}$. On the other hand, an abelian automorphic $\Pi^{\prime}$ is an Eisenstein representation. Shahidi's calculation of Whittaker coefficients for Eisenstein series is the third main ingredient of the proof. It allows us to identify the Whittaker period $p\left(\Pi^{\prime}\right)$ with a motivic period attached to the collection of $\chi_{i}$. A similar identification was already exploited in [34], for Eisenstein classes attached to other parabolic subgroups. In our case, the motivic expression for $p\left(\Pi^{\prime}\right)$ exactly cancels the terms in the expression calculated in [20], yielding an expression (Theorem 6.7) for $p(\Pi)$ solely in terms of Petersson norms of holomorphic forms on Shimura varieties, multiplied by purely archimedean invariants that have not yet been calculated explicitly.

Theorem 1.4. Let $\Pi$ be a cuspidal automorphic representation of $\mathrm{GL}_{n}\left(\mathbb{A}_{\mathcal{K}}\right)$, which can be obtained by base change from unitary groups of all signatures (i.e., more precisely, satisfies Hypothesis 4.19). Assume that $\Pi$ is cohomological with respect to the irreducible, finite-dimensional, algebraic representation $E_{\mu}$ of highest weight $\mu_{G}=$ $\left(\mu_{1}, \ldots, \mu_{n} ;-\mu_{n}, \ldots,-\mu_{1}\right)$, and suppose that $\mu_{i}-\mu_{i+1} \geqslant 2$ for all $i$, so that there is a highest weight $\lambda_{G}=\left(\lambda ; \lambda^{\mathrm{v}}\right)$ for $G_{\infty}^{\prime}$ and an integer $m>0$ such that $s_{0}=\frac{1}{2}+m$ is a critical value of $L\left(s, \Pi \otimes \Pi^{\prime}\right)$ for any cuspidal automorphic representation $\Pi^{\prime}$ of $\mathrm{GL}_{n-1}\left(\mathbb{A}_{\mathcal{K}}\right)$, which is cohomological with respect to $E_{\lambda}$. Then there is a non-zero constant $Z\left(\Pi_{\infty}\right)$, which depends only on the local representation $\Pi_{\infty}$ and such that

$$
p(\Pi) \sim_{\mathcal{K} E(\Pi)} Z\left(\Pi_{\infty}\right) \prod_{j=1}^{n-1} P^{(j)}(\Pi) .
$$

The constant $Z\left(\Pi_{\infty}\right)$, like the expression $u\left(m, \Pi_{\infty}, \Pi_{\infty}^{\prime}\right)$ in Theorem 1.3 , is well defined up to a non-zero scalar in $\mathcal{K}$. It should be mentioned that the latter theorem (or equivalently Theorem 6.7) is conditional on a global non-vanishing hypothesis. Verifying this hypothesis for the central critical value appears to be extremely difficult in general, 
but fortunately can always be satisfied provided that the infinitesimal character of $\Pi$ is sufficiently regular.

Putting together Theorems 1.3 and 1.4 with the results of [20], we easily obtain the main theorem, with the constant $u\left(m, \Pi_{\infty}, \Pi_{\infty}^{\prime}\right)=p\left(m, \Pi_{\infty}, \Pi_{\infty}^{\prime}\right) \cdot Z\left(\Pi_{\infty}\right) \cdot Z\left(\Pi_{\infty}^{\prime}\right)$. The individual terms in the product depend on normalizations of archimedean Whittaker models, as do the Whittaker periods $p(\Pi)$ and $p\left(\Pi^{\prime}\right)$ themselves, but the product is independent of normalizations, up to $\mathcal{K}^{\times}$. Following the announcement of these results, Jie Lin has identified $u\left(m, \Pi_{\infty}, \Pi_{\infty}^{\prime}\right)$ as the explicit power of $2 \pi i$ predicted by the automorphic version of Deligne's conjecture described in $\S 4.2$; see Theorem 6.11 for a precise statement.

In the absence of the regularity hypothesis of 1.2 , we can prove a rather different kind of result, using Zhang's recent proof of a version of Neal Harris's Ichino-Ikeda conjecture for unitary groups [19]. Suppose that $\Pi$ and $\Pi^{\prime}$ satisfy the hypotheses of [51]; in particular, $\Pi$ and $\Pi^{\prime}$ are both supercuspidal at some prime of $\mathcal{K}$ split over $\mathbb{Q}$. Then the conclusion of Theorem 1.3 holds, with $p(\Pi)$ and $p\left(\Pi^{\prime}\right)$ replaced by the values at $s=1$ of the adjoint $L$-functions of $\pi$ and $\pi^{\prime}$ respectively, and with slightly different archimedean factors (See Corollary 6.25).

Theorem 1.5. Let $\Pi$ and $\Pi^{\prime}$ be cuspidal automorphic representations of $\mathrm{GL}_{n}\left(\mathbb{A}_{\mathcal{K}}\right)$ and $\mathrm{GL}_{n-1}\left(\mathbb{A}_{\mathcal{K}}\right)$ which are cohomological with respect to $E_{\mu}$ and $E_{\lambda}$, respectively, assumed to satisfy the equivalent conditions of Lemma 2.1. We assume that $\Pi=B C(\pi)$ and $\Pi^{\prime}=$ $B C\left(\pi^{\prime}\right)$ are obtained by base change from definite unitary groups and that the irreducible unitary cuspidal automorphic representations $\pi$ and $\pi^{\prime}$ satisfy the hypotheses of [51] (more precisely, Hypothesis 6.12). Then, for every critical point $s_{0}=\frac{1}{2}+m$ of $L(s, \Pi \times$ $\left.\Pi^{\prime}\right)$ with $m \geqslant 0$, there are, a non-zero complex constant $a\left(m, \Pi_{\infty}, \Pi_{\infty}^{\prime}\right)$, depending only on the archimedean components of $\Pi$ and $\Pi^{\prime}$, and an integer $a(n)$ only depending on $n$, such that

$$
L\left(\frac{1}{2}+m, \Pi_{f} \times \Pi_{f}^{\prime}\right) \sim_{\mathcal{K} \mathbb{Q}(\pi) \mathbb{Q}\left(\pi^{\prime}\right)} a\left(m, \Pi_{\infty}, \Pi_{\infty}^{\prime}\right) \mathcal{G}(\varepsilon \mathcal{K}, f)^{a(n)} L\left(1, \pi_{f}, A d\right) L\left(1, \pi_{f}^{\prime}, A d\right),
$$

where $\sim \mathcal{K} \mathbb{Q}(\pi) \mathbb{Q}\left(\pi^{\prime}\right)$ means up to multiplication by an element in the composition of number fields $\mathcal{K} \mathbb{Q}(\pi) \mathbb{Q}\left(\pi^{\prime}\right)$. Equivalently, for every critical point $s_{0}=n-1+m$ of $L(s, R(M(\Pi) \otimes$ $\left.\left.M\left(\Pi^{\prime}\right)\right)\right)$ with $m \geqslant 0$,

$$
\begin{aligned}
& L\left(n-1+m, R\left(M(\Pi) \otimes M\left(\Pi^{\prime}\right)\right)\right) \\
& \quad \sim_{\mathcal{K} \mathbb{Q}(\pi) \mathbb{Q}\left(\pi^{\prime}\right)} a\left(m, \Pi_{\infty}, \Pi_{\infty}^{\prime}\right) \mathcal{G}(\varepsilon \mathcal{K}, f)^{a(n)} L\left(1, \pi_{f}, A d\right) L\left(1, \pi_{f}^{\prime}, A d\right) .
\end{aligned}
$$

While Deligne's conjecture provides an expression of the critical values in terms of Deligne periods, which are defined in terms of motives, and whose status is therefore at least partially hypothetical, the Petersson norms are invariants of authentically arithmetic objects. The purpose of $\S 4$ is to provide plausible reasons to identify Deligne's periods in our situation with the expressions derived using [20]. It should be noted that [20] was written with applications to self-dual cohomological automorphic representations in mind, although many of the results are valid more generally. For this reason, the standing self-duality hypothesis of [20] is sometimes invoked tacitly, simplifying some statements but making it difficult for the reader (including the author of [20], at more than 15 years' 
distance) to determine which statements need to be modified to treat the general case. We have therefore attempted in $\S 4$, and especially in 4.4 , to provide complete statements with precise parameters. The authors hope that definitive statements, over general CM fields (i.e., totally imaginary quadratic extensions of totally real fields), will be available in the near future.

For the convenience of the reader, we wrote 1.2 from the automorphic point of view as well as from the motivic point of view. It is shown in Optimistic Comparison 4.32 that the period that appears in Theorem 1.2 , namely

$$
\prod_{j=1}^{n-1} P^{(j)}(\Pi) \prod_{k=1}^{n-2} P^{(k)}\left(\Pi^{\prime}\right),
$$

is at least formally consistent with Deligne's conjecture, provided that the Hodge types of the motives attached to $\Pi$ and $\Pi^{\prime}$ satisfy the relations derived from Lemma 2.1. The article [23] computes Deligne periods for general Rankin-Selberg products and obtains different products of the same basic invariants $P^{(j)}(\Pi)$ and $P^{(k)}\left(\Pi^{\prime}\right)$ (under the identifications of Optimistic Comparison 4.32). The final section explains how one might hope to express the critical values of general Rankin-Selberg products, using normalized Rankin-Selberg integrals of Eisenstein cohomology classes.

The Whittaker period invariants $p(\Pi)$ are defined by comparing two rational structures on $\Pi_{f}$, one coming from the global realization of $\Pi_{f}$ in the cohomology of the locally symmetric space $S_{n}$ attached to $\mathrm{GL}_{n}$, and the other from the Whittaker model. It is well known, however, that $\Pi_{f}$ occurs in a range of cohomological degrees $b_{n} \leqslant i \leqslant c_{n}$, and each one gives rise to one (or more) period invariants. Our $p(\Pi)$ is attached to the lowest cohomological degree $b_{n}$; the proof of Theorem 1.3 is based on the numerical coincidence that $b_{n}+b_{n-1}-1$ equals the dimension of $S_{n-1}$. In [45], Urban showed that, when $n=2$, the Whittaker period attached to $\Pi$ in the top cohomological degree $c_{2}$ is related to a non-critical special value of one of the two Asai $L$-functions attached to $\Pi$. It can be deduced from the arguments of [51] and the work of Jacquet, Rallis, and Flicker cited there that the phenomenon Urban discovered remains valid for general $n$; this is the subject of our forthcoming article with Lapid [14].

\section{Unitary groups and base change}

\subsection{Some standing assumptions and notation}

2.1.1. Number fields. Let $\mathcal{K} / \mathbb{Q}$ denote an imaginary quadratic extension of $\mathbb{Q}$. We write $S(\mathcal{K})$ for the set of places of $\mathcal{K}$, and $S$ for the set of places of $\mathbb{Q}$. The ring of adeles over $\mathcal{K}$ (respectively, over $\mathbb{Q}$ ) is denoted $\mathbb{A}_{\mathcal{K}}$ (respectively, $\mathbb{A}$ ). The ring of integers of $\mathcal{K}$ is denoted $\mathcal{O}$, and we let $\mathfrak{D}_{\mathcal{K}}$ be the different of $\mathcal{K} / \mathbb{Q}$, i.e., $\mathfrak{D}_{\mathcal{K}}^{-1}=\left\{x \in \mathcal{K}: \operatorname{Tr}_{\mathcal{K} / \mathbb{Q}}(x \mathcal{O}) \subset\right.$ $\mathbb{Z}$ \}, where $\operatorname{Tr}_{\mathcal{K} / \mathbb{Q}}$ is the trace map from $\mathcal{K}$ to $\mathbb{Q}$. The normalized absolute value of $\mathbb{A} \mathcal{K}$ is denoted $\|\cdot\|$. We extend the Hecke character $\varepsilon \mathcal{K}: \mathbb{Q}^{\times} \backslash \mathbb{A}^{\times} \rightarrow \mathbb{C}^{\times}$, associated to the extension $\mathcal{K} / \mathbb{Q}$ via class field theory, to a Hecke character $\eta: \mathcal{K}^{\times} \backslash \mathbb{A}_{\mathcal{K}}^{\times} \rightarrow \mathbb{C}^{\times}$. It is easy to see that $\eta$ is unitary and $\eta_{\infty}(z)=z^{t} \bar{z}^{-t}$ for a certain $t=t(\eta) \in \frac{1}{2}+\mathbb{Z}$.

For convenience, we will also fix, once and for all, a non-trivial, continuous, additive character $\psi: \mathcal{K} \backslash \mathbb{A} \mathcal{K} \rightarrow \mathbb{C}^{\times}$. To this end, let $\psi_{\mathbb{Q}}$ be the additive character of $\mathbb{Q} \backslash \mathbb{A}$, as in Tate's thesis, namely, $\psi_{\mathbb{Q}}(x)=e^{2 \pi i \Lambda(x)}$ with the $\Lambda=\sum_{p \leqslant \infty} \Lambda_{p}$, where $\Lambda_{\infty}(x)=-x$ 
for $x \in \mathbb{R}$ and $\Lambda_{p}\left(x_{p}\right), x_{p} \in \mathbb{Q}_{p}$, is the finite, negative tail of $x_{p}$ 's $p$-adic expansion. That is, if $x_{p}=\sum_{j \geqslant v} a_{j} p^{j}$, then $\Lambda_{p}\left(x_{p}\right)=\sum_{j=v}^{-1} a_{j} p^{j}$. If we write $\psi_{\mathbb{Q}}=\psi_{\mathbb{R}} \otimes \otimes_{p} \psi_{\mathbb{Q}_{p}}$, then $\psi_{\mathbb{R}}(x)=e^{-2 \pi i x}$, and $\psi_{\mathbb{Q}_{p}}$ is trivial on $\mathbb{Z}_{p}$ and non-trivial on $p^{-1} \mathbb{Z}_{p}$.

Now, we define $\psi:=\psi_{\mathbb{Q}} \circ \operatorname{Tr} r_{\mathcal{K} / \mathbb{Q}}$. If $\psi=\otimes_{w} \psi_{w}$, then the local characters are determined analogously. In particular, if $\mathfrak{D}_{\mathcal{K}}=\prod_{\wp} \wp^{r_{\wp}}$, the product running over all prime ideals $\wp \subset \mathcal{O}$, then the conductor of the local character $\psi_{w}$ is $\wp_{w}^{-r_{\wp}}$, i.e., $\psi_{w}$ is trivial on $\wp_{w}^{-r_{\wp}}$ and non-trivial on $\wp_{w}^{-r_{\wp}-1}$. We let $S(\psi)=\left\{\wp \mid \mathfrak{D}_{\mathcal{K}}\right\}$.

2.1.2. Hermitian spaces and unitary groups. We let $V$ be an $n$-dimensional Hermitian space over $\mathcal{K}$, relative to the non-trivial element in $\operatorname{Gal}(\mathcal{K} / \mathbb{Q})$. Depending on the parity of $n$, we define

$$
\gamma:= \begin{cases}\mathbf{1} & \text { if } n \text { is even } \\ \eta & \text { if } n \text { is odd }\end{cases}
$$

Hence, $\gamma_{\infty}(z)=z^{c} \bar{z}^{-c}$, where $c=0$, if $n$ is even and $c=t \in \frac{1}{2}+\mathbb{Z}$, if $n$ is odd.

We let $V^{\prime} \subset V$ be a subspace of codimension 1, on which the restriction of the Hermitian form is non-degenerate so that $V=V^{\prime} \oplus V^{\prime \perp}$. At infinity, we want $V$ (and $V^{\prime}$ ) to be definite.

We let $H:=U(V)$ and $H^{\prime}:=U\left(V^{\prime}\right)$ be the corresponding unitary groups over $\mathbb{Q}$. In particular, $H(\mathbb{R}) \cong U(n)$, the compact unitary group of rank $n$, and similarly $H^{\prime}(\mathbb{R}) \cong$ $U(n-1)$.

Furthermore, we let $G:=\mathrm{GL}_{n} / \mathcal{K}$ and $G^{\prime}:=\mathrm{GL}_{n-1} / \mathcal{K}$. We also define real Lie groups $K:=\mathrm{GU}(V)(\mathbb{R})$ (respectively, $K^{\prime}:=\mathrm{GU}\left(V^{\prime}\right)(\mathbb{R})$ ), which we view inside $G(\mathbb{C})$ (respectively, $G^{\prime}(\mathbb{C})$ ) by their natural embedding.

The Lie algebra of a real Lie group is denoted by the same (but gothic) lower-case letter.

2.1.3. Finite-dimensional representations of real groups. Let $E_{\mu}^{\text {unt }}$ (respectively, $\left.E_{\lambda}^{\text {unt }}\right)$ ) be an irreducible, finite-dimensional, algebraic representation of $H(\mathbb{R})$ $\left(\right.$ respectively, $\left.H^{\prime}(\mathbb{R})\right)$ on a complex vector space. Having fixed a maximal $\mathbb{Q}$-split torus in $H(\mathbb{C}) \cong \mathrm{GL}_{n}(\mathbb{C})$ (respectively, $H^{\prime}(\mathbb{C}) \cong \mathrm{GL}_{n-1}(\mathbb{C})$ ) and an ordering on the set of its dominant algebraic characters, we may think of $E_{\mu}^{\text {unt }}$ (respectively, $E_{\lambda}^{\text {unt }}$ )) as being given by its highest weight $\mu$ (respectively, $\lambda$ ). We may arrange that $\mu=\left(\mu_{1}, \ldots, \mu_{n}\right)$, where $\mu_{1} \geqslant \cdots \geqslant \mu_{n}, \mu_{i} \in \mathbb{Z}$ for all $1 \leqslant i \leqslant n$, and similarly $\lambda=\left(\lambda_{1}, \ldots, \lambda_{n-1}\right)$ with $\lambda_{1} \geqslant \cdots \geqslant \lambda_{n-1}, \lambda_{i} \in \mathbb{Z}$ for all $1 \leqslant i \leqslant n-1$.

Furthermore, we write $E_{\mu}^{\text {unt }} \otimes E_{\mu^{v}}^{\text {unt }} \quad$ (respectively, $\quad E_{\lambda}^{\text {unt }} \otimes E_{\lambda^{v}}^{\text {unt }}$ ) for the irreducible, finite-dimensional, algebraic representation of the real Lie group $G(\mathbb{C})$ (respectively, $G^{\prime}(\mathbb{C})$ ) of highest weight $\left(\mu, \mu^{\mathrm{v}}\right)$ (respectively, $\left(\lambda, \lambda^{\mathrm{v}}\right)$ ). (Here, the check $\mathrm{v}$ denotes taking the contragredient representation.) It is isomorphic to its complex conjugate contragredient.

Lemma 2.1 (See, e.g., [11, Theorem 8.1.1]). With the above notation, the following assertions are equivalent.

(1) $\mu_{1} \geqslant-\lambda_{n-1} \geqslant \mu_{2} \geqslant-\lambda_{n-2} \geqslant \cdots \geqslant-\lambda_{1} \geqslant \mu_{n}$.

(2) $\operatorname{Hom}_{H^{\prime}(\mathbb{C})}\left(E_{\mu}^{\mathrm{unt}} \otimes E_{\lambda}^{\mathrm{unt}}, \mathbb{C}\right) \neq 0$. 
If any of these two conditions is satisfied, then also $H_{o m} m_{G^{\prime}(\mathbb{C})}\left(\left(E_{\mu}^{\mathrm{unt}} \otimes E_{\mu^{\mathrm{v}}}^{\mathrm{unt}}\right) \otimes\left(E_{\lambda}^{\mathrm{unt}} \otimes\right.\right.$ $\left.\left.E_{\lambda^{\mathrm{v}}}^{\mathrm{unt}}\right), \mathbb{C}\right) \neq 0$.

\subsection{Base change}

We let $\pi$ (respectively, $\pi^{\prime}$ ) be an irreducible, unitary cuspidal automorphic representation of $H(\mathbb{A})$ (respectively, $H^{\prime}(\mathbb{A})$ ). We shall always assume that $\pi$ (respectively, $\pi^{\prime}$ ) is cohomological with respect to $E_{\mu}^{\mathrm{unt}}\left(\right.$ respectively, $\left.\left.E_{\lambda}^{\mathrm{unt}}\right)\right)$. Since $H(\mathbb{R})$ and $H^{\prime}(\mathbb{R})$ are both compact and connected, this simply means that $\pi_{\infty} \cong E_{\mu^{v}}^{\text {unt }}$ and $\pi_{\infty}^{\prime} \cong E_{\lambda^{v}}^{\text {unt }}$. We let $S(\pi)$ (respectively, $S\left(\pi^{\prime}\right)$ ) be the set of all finite places of $\mathbb{Q}$, where $\pi$ (respectively, $\pi^{\prime}$ ) ramifies, together with the infinite place of $\mathbb{Q}$. The following is essentially a theorem of Labesse.

Theorem 2.2 (Base change). There exist irreducible automorphic representations $\Pi$ of $G\left(\mathbb{A}_{\mathcal{K}}\right)$ and $\Pi^{\prime}$ of $G^{\prime}\left(\mathbb{A}_{\mathcal{K}}\right)$, which are of the form

$$
\Pi \cong \Pi_{1} \boxplus \cdots \boxplus \Pi_{s} \quad \text { and } \quad \Pi^{\prime} \cong \Pi_{1}^{\prime} \boxplus \cdots \boxplus \Pi_{r}^{\prime},
$$

where $\Pi_{j}$ (respectively, $\Pi_{j}^{\prime}$ ) is a square-integrable automorphic representation of $\mathrm{GL}_{s_{j}}\left(\mathbb{A}_{\mathcal{K}}\right)$ (respectively, $\mathrm{GL}_{r_{j}}\left(\mathbb{A}_{\mathcal{K}}\right)$ ), for all $j$, such that the following hold.

- For all $p \notin S(\pi)$, the representation $\Pi_{p}:=\otimes_{w \mid p} \Pi_{w}$ is the local base change of $\pi_{p}$.

- The archimedean component $\Pi_{\infty}$ of $\Pi$ is cohomological with respect to $E_{\mu}^{\mathrm{unt}} \otimes E_{\mu^{\mathrm{v}}}^{\mathrm{unt}}$, i.e., $H^{*}\left(\mathfrak{g}, K, \Pi_{\infty} \otimes\left(E_{\mu}^{\mathrm{unt}} \otimes E_{\mu^{\mathrm{v}}}^{\mathrm{unt}}\right)\right) \neq 0$.

And analogously, the following hold.

- For all $p \notin S\left(\pi^{\prime}\right)$, the representation $\Pi_{p}^{\prime}:=\otimes_{w \mid p} \Pi_{w}^{\prime}$ is the local base change of $\pi_{p}^{\prime}$.

- The archimedean component $\Pi_{\infty}^{\prime}$ of $\Pi^{\prime}$ is cohomological with respect to $E_{\lambda}^{\mathrm{unt}} \otimes E_{\lambda^{v}}^{\mathrm{unt}}$, i.e., $H^{*}\left(\mathfrak{g}^{\prime}, K^{\prime}, \Pi_{\infty}^{\prime} \otimes\left(E_{\lambda}^{\mathrm{unt}} \otimes E_{\lambda^{\mathrm{v}}}^{\mathrm{unt}}\right)\right) \neq 0$.

If all the isobaric summands $\Pi_{j}$ (respectively, $\Pi_{j}^{\prime}$ ) are cuspidal, then $\Pi_{\infty}$ (respectively, $\Pi_{\infty}^{\prime}$ ) is the local base change of $\pi_{\infty}$ (respectively, $\left.\pi_{\infty}^{\prime}\right)$.

Proof. This is essentially due to Labesse; see [31, Corollary 5.3], as well as [30]. Strictly speaking, the proof given in the aforementioned reference only works for totally real number fields $F$ different from $\mathbb{Q}$. However, there is no doubt that the theorem holds in the above form. See [31, Remarque 5.2] and also [30].

\subsection{Descent to unitary groups and base change}

When $B C(\pi)$ is cuspidal, it is expected, and has been proved in a great many cases (see for example [31]) that $\pi$ occurs with multiplicity 1 in the discrete spectrum of $H$. Moreover, if $H^{b}$ is any inner form of $H$, then $\Pi$ descends to an $L$-packet $\left\{\pi^{b}\right\}$ of discrete (cuspidal) automorphic representations of $H^{b}$ whose archimedean components lie in the discrete series; again it is expected that each member of the packet occurs with multiplicity 1.

Suppose that $H^{\mathrm{b}}(\mathbb{R}) \cong U(r, s)$, the unitary group of signature $(r, s)$. Then any $\pi^{\mathrm{b}}$ on $H^{b}$ whose base change is $B C(\pi)$, and whose archimedean component is a holomorphic (discrete series) representation, will be denoted $\pi_{r, s}$. Thus, $\pi=\pi_{n, 0}$ or $\pi_{0, n}$. It does not matter whether $H$ is positive or negative definite; but when $r s>0$, the difference 
between $\pi_{r, s}$ and $\pi_{s, r}$ needs to be respected, because they define holomorphic automorphic forms on non-isomorphic Shimura varieties (attached to the similitude groups containing $\left.H^{b}\right)$. The normalizations relevant to these Shimura varieties can be found in [20].

To $\pi_{r, s}$ we associate a period invariant $P^{(s)}(B C(\pi)) \in \mathbb{C}^{\times}$, as in $[23, \S 4.2]$. Roughly speaking, $P^{(s)}(B C(\pi))$ is the square of the Petersson norm of an arithmetically normalized holomorphic automorphic form in $\pi_{r, s}$. Precise definitions are in [20]; see also $\S 4.4$ below, especially Hypothesis 4.19, as well as Remark 6.9.

\subsection{The cuspidal automorphic representation $\Pi$}

Recall the automorphic representation $B C(\pi)$ from Theorem 2.2. We make the additional assumption that it is cuspidal, and take an arbitrary, but henceforth fixed, integer $m \in \mathbb{Z}$. We define

$$
\Pi:=B C(\pi)\|\cdot\|^{\mathrm{m}},
$$

suppressing its dependence on $\mathrm{m}$. By assumption, $\Pi$ is cuspidal automorphic, and hence $\Pi$ is automatically globally $\psi^{-1}$-generic. We denote by $W\left(\Pi_{f}\right)$ the corresponding Whittaker model of $\Pi_{f}$, again suppressing the dependence on the fixed additive character $\psi^{-1}$. By Theorem 2.2, $\Pi_{\infty}$ is cohomological with respect to the finite-dimensional algebraic representation

$$
E_{\mu}:=\left(E_{\mu}^{\mathrm{unt}} \cdot \operatorname{det}^{-\mathrm{m}}\right) \otimes\left(E_{\mu^{v}}^{\mathrm{unt}} \cdot \operatorname{det}^{-\mathrm{m}}\right)=E_{\mu-\mathrm{m}}^{\mathrm{unt}} \otimes E_{\mu^{v}-\mathrm{m}}^{\mathrm{unt}}
$$

of $G(\mathbb{C})$ of highest weight $\mu_{G}:=\left(\mu-\mathrm{m}, \mu^{\mathrm{v}}-\mathrm{m}\right)$; hence, we have

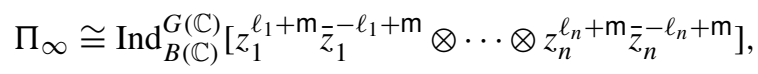

where $B=T N$ is the standard Borel subgroup of $G$, and

$$
\ell_{j}=-\mu_{n-j+1}+\frac{n+1}{2}-j .
$$

In analogy to the case of unitary groups, we let $S(\Pi)$ be the set of finite places of $\mathcal{K}$, where $\Pi$ ramifies, together with the infinite place of $\mathcal{K}$.

\subsection{The abelian automorphic representation $\Pi^{\prime}$}

Now recall the automorphic representations $B C\left(\pi^{\prime}\right)$ from Theorem 2.2 . We set $\Pi^{\prime}:=$ $B C\left(\pi^{\prime}\right)$, and make the following additional assumption. We suppose that $\Pi^{\prime} \cong \Pi_{1}^{\prime} \boxplus$ $\cdots \boxplus \Pi_{r}^{\prime}$ is abelian (and therefore not square integrable), i.e.,

$$
\Pi_{j}^{\prime}=B C\left(\chi_{j}\right) \cdot \gamma \quad 1 \leqslant j \leqslant n-1,
$$

where $B C\left(\chi_{j}\right)$ is the base change of an algebraic Hecke character $\chi_{j}: U(1)(\mathbb{Q}) \backslash U(1)(\mathbb{A}) \rightarrow$ $\mathbb{C}^{\times}$. More explicitly, $B C\left(\chi_{j}\right)(g)=\chi_{j}(g / \bar{g})$ for all $g \in \mathbb{A}_{\mathcal{K}}^{\times}$, where $\bar{g}$ denotes conjugation relative to the non-trivial element in $\operatorname{Gal}(\mathcal{K} / \mathbb{Q})$. At the archimedean place of $\mathbb{Q}$ (respectively, $\mathcal{K})$ we obtain $\chi_{j, \infty}\left(e^{i \theta}\right)=e^{i k_{j} \theta}, e^{i \theta} \in U(1)$, (respectively, $B C\left(\chi_{j}\right)_{\infty}(z)=$ $\left.z^{k_{j}} \bar{z}^{-k_{j}}, z \in \mathbb{C}^{\times}\right)$for $k_{j} \in \mathbb{Z}$. Therefore,

$$
\Pi_{\infty}^{\prime} \cong \operatorname{Ind}_{B^{\prime}(\mathbb{C})}^{G^{\prime}(\mathbb{C})}\left[z_{1}^{k_{1}+c} \bar{z}_{1}^{-k_{1}-c} \otimes \cdots \otimes z_{n-1}^{k_{n-1}+c} \bar{z}_{n-1}^{-k_{n-1}-c}\right]
$$


where $B^{\prime}=T^{\prime} N^{\prime}$ is the standard Borel subgroup of $G^{\prime}$ and so, since $\Pi_{\infty}^{\prime}$ is cohomological with respect to the finite-dimensional algebraic representation

$$
E_{\lambda}:=E_{\lambda}^{\mathrm{unt}} \otimes E_{\lambda^{\mathrm{v}}}^{\mathrm{unt}}
$$

of $G^{\prime}(\mathbb{C})$ of highest weight $\lambda_{G^{\prime}}:=\left(\lambda, \lambda^{v}\right)$, necessarily,

$$
k_{j}=-\lambda_{n-j}+\frac{n}{2}-j-c .
$$

Hence, we can further see that $k_{1}>k_{2}>\cdots>k_{n-1}$. In particular, this implies, using Shahidi [42, Proposition 7.1.3, Theorem 3.5.12 and Remark 3.5.14] that $\Pi^{\prime}$ is globally $\psi$-generic, i.e., the $\psi$-Whittaker coefficient of $\Pi^{\prime}$ defines a non-vanishing intertwining $\Pi^{\prime} \hookrightarrow \operatorname{Ind}_{N^{\prime}(\mathbb{A})}^{G^{\prime}(\mathbb{A})}[\psi]$ (unnormalized induction). As for $\Pi$, we denote by $W\left(\Pi_{f}^{\prime}\right)$ the corresponding Whittaker model of $\Pi_{f}^{\prime}$, and let $S\left(\Pi^{\prime}\right)$ be the set of finite places of $\mathcal{K}$, where $\Pi^{\prime}$ ramifies, together with the infinite place of $\mathcal{K}$.

We will make the following assumption, valid throughout the paper.

Hypotheses 2.3. The highest weights $\mu$ and $\lambda$ satisfy

$$
\mu_{1} \pm \mathrm{m} \geqslant-\lambda_{n-1} \geqslant \mu_{2} \pm \mathrm{m} \geqslant-\lambda_{n-2} \geqslant \cdots \geqslant-\lambda_{1} \geqslant \mu_{n} \pm \mathrm{m} .
$$

Lemma 2.1 then guarantees that $\operatorname{Hom}_{G^{\prime}(\mathbb{C})}\left(E_{\mu} \otimes E_{\lambda}, \mathbb{C}\right) \neq 0$, which will be important from $\S 3.6$ on.

\subsection{An action of $\operatorname{Aut}(\mathbb{C})$}

For a moment, let $k \geqslant 1$ be any integer, and let $E$ be an irreducible, finite-dimensional, algebraic representation of $\mathrm{GL}_{k} / \mathcal{K}$. As a representation of the real Lie group $\mathrm{GL}_{k}(\mathbb{C}), E$ factors as $E=E_{\iota} \otimes E_{\bar{\iota}}$, for a fixed embedding $\iota: \mathcal{K} \hookrightarrow \mathbb{C}$. For a given $\sigma \in \operatorname{Aut}(\mathbb{C})$, we define the $\mathrm{GL}_{k}(\mathbb{C})$-representation

$$
{ }^{\sigma} E:=E_{\sigma \circ \iota} \otimes E_{\overline{\sigma \circ \iota}} .
$$

Hence, ${ }^{\sigma} E$ is identical to $E$, if $\sigma \circ \iota=\iota$, and it is the representation ${ }^{\sigma} E=E_{\bar{\iota}} \otimes E_{\iota}$, otherwise, i.e., if $\sigma \circ \iota=\bar{\imath}$. Furthermore, for a representation of $\mathrm{GL}_{k}(\mathbb{C})$, which is induced from the Borel subgroup $B_{k}=T_{k} N_{k}$ of $\mathrm{GL}_{k}, \Delta_{\infty}=\operatorname{Ind}_{B_{k}(\mathbb{C})}^{\mathrm{GL}_{k}(\mathbb{C})}\left[\omega_{1} \otimes \cdots \otimes \omega_{k}\right]$, we let

$$
{ }^{\sigma} \Delta_{\infty}:=\operatorname{Ind}_{B_{k}(\mathbb{C})}^{\mathrm{GL}_{k}(\mathbb{C})}\left[{ }^{\sigma} \omega_{1} \otimes \cdots \otimes{ }^{\sigma} \omega_{k}\right] .
$$

Note that, if $\Delta_{\infty}$ is cohomological with respect to $E$, then ${ }^{\sigma} \Delta_{\infty}$ is cohomological with respect to ${ }^{\sigma} E$.

If $\Delta_{f}$ (respectively, $\Delta_{w}, w \in S(\mathcal{K})_{f}$ ) is an irreducible representation of $\operatorname{GL}_{k}\left(\mathbb{A}_{f}\right)$ (respectively, $\mathrm{GL}_{k}\left(\mathcal{K}_{w}\right)$ ), then we denote by ${ }^{\sigma} \Delta_{f}$ (respectively, ${ }^{\sigma} \Delta_{w}$ ) the representation defined by Waldspurger in [46, I.1]. Analogously, if $\delta_{\infty}$ is an irreducible representation of $H(\mathbb{R})$ or $H^{\prime}(\mathbb{R})$, we let ${ }^{\sigma} \delta_{\infty}:=\delta_{\infty}$, since there is only the identity embedding of $\mathbb{Q}$ into $\mathbb{C}$. For representations $\delta_{f}$ (respectively, $\delta_{p}, p \in S_{f}$ ) of $H\left(\mathbb{A}_{f}\right)$ or $H^{\prime}\left(\mathbb{A}_{f}\right)$ (respectively, $H\left(\mathbb{Q}_{p}\right)$ or $H^{\prime}\left(\mathbb{Q}_{p}\right)$ ), we use again the definition of Waldspurger, [46, I.1]. 
If $\delta$ is any of the above representations, we let $\mathfrak{S}(\delta):=\left\{\left.\sigma \in \operatorname{Aut}(\mathbb{C})\right|^{\sigma} \delta \cong \delta\right\}$, and define the rationality field of $\delta$ to be

$$
\mathbb{Q}(\delta):=\{z \in \mathbb{C} \mid \sigma(z)=z \quad \forall \sigma \in \mathfrak{S}(\delta)\} .
$$

See again Waldspurger, [46, I.1]. We say that a representation $\delta$ is defined over a field $F$ if there is an $F$-subspace $\delta_{0}$ of the representation space of $\delta$ which is invariant under the group action and such that the natural map $\delta_{0} \otimes_{F} \mathbb{C} \rightarrow \delta$ is an isomorphism.

Proposition 2.4. For all $\sigma \in \operatorname{Aut}(\mathbb{C})$, there is an isomorphism

$$
{ }^{\sigma} \Pi^{\prime} \cong \boxplus_{j=1}^{n-1 \sigma} B C\left(\chi_{j}\right)^{\sigma}\left(\gamma\|\cdot\|^{\frac{n-2 j}{2}}\right)\|\cdot\|^{\frac{2 j-n}{2}} .
$$

In other words, ${ }^{\sigma} \Pi^{\prime}$ is again the isobaric automorphic sum of Hecke characters. Similarly, ${ }^{\sigma} \Pi$ is again a cuspidal automorphic representation.

Proof. The first assertion results from a straightforward calculation, using the fact that, since $B C\left(\chi_{j}\right)$ and $\gamma\|\cdot\|^{\frac{n-2 j}{2}}$ are both algebraic Hecke characters, so are ${ }^{\sigma} B C\left(\chi_{j}\right)$ and $\sigma\left(\gamma\|\cdot\|^{\frac{n-2 j}{2}}\right)$. The last assertion on $\Pi$ can be shown as follows. By [5, Theorem 3.13], there is a cuspidal automorphic representation $\Xi$ of $G\left(\mathbb{A}_{\mathcal{K}}\right)$, cohomological with respect to ${ }^{\sigma} E_{\mu}$ and such that $\Xi_{f} \cong{ }^{\sigma} \Pi_{f}$. As a cohomological cuspidal automorphic representation has an essentially tempered archimedean component, it follows from the classification of cohomological representations of $G(\mathbb{C})$ that necessarily $\Xi_{\infty} \cong{ }^{\sigma} \Pi_{\infty}$.

Proposition 2.5. The finite part $\Pi_{f}$ of $\Pi=B C(\pi)\|\cdot\|^{\mathrm{m}} \quad$ (respectively, $\Pi_{f}^{\prime}$ of $\Pi^{\prime}=$ $B C\left(\pi^{\prime}\right)$ ) is defined over its rationality field $\mathbb{Q}\left(\Pi_{f}\right)$ (respectively, $\mathbb{Q}\left(\Pi_{f}^{\prime}\right)$ ), which is an extension of $\mathbb{Q}\left(E_{\mu}\right)$ (respectively, of $\mathbb{Q}\left(E_{\lambda}\right)$ ). This structure is unique up to homotheties. Both rationality fields are number fields. As a representation of $\mathrm{GL}_{k}(\mathcal{K}) \subset \mathrm{GL}_{k}(\mathbb{C})$, an irreducible, finite-dimensional, algebraic representation $E$ of $\mathrm{GL}_{k}(\mathbb{C})$ is defined over $\mathbb{Q}(E)$.

Proof. The fields $\mathbb{Q}\left(\Pi_{f}\right)$ and $\mathbb{Q}\left(\Pi_{f}^{\prime}\right)$ extend $\mathbb{Q}\left(E_{\mu}\right)$, respectively $\mathbb{Q}\left(E_{\lambda}\right)$, because of Strong Multiplicity One for isobaric automorphic representations. Here, we use Proposition 2.4. Moreover, as a consequence of $\left[5\right.$, Theorem 3.13], $\mathbb{Q}\left(\Pi_{f}\right)$ is a number field. (For a detailed proof, one may also have a look at [15, Theorem 8.1].) By Proposition 2.4,

$$
\bigcap_{j=1}^{n-1} \mathfrak{S}\left(B C\left(\chi_{j}\right)_{f} \gamma_{f}\|\cdot\|_{f}^{\frac{n-2 j}{2}}\right) \subseteq \mathfrak{S}\left(\Pi_{f}^{\prime}\right),
$$

so $\mathbb{Q}\left(\Pi_{f}^{\prime}\right) \subseteq \prod_{j=1}^{n-1} \mathbb{Q}\left(B C\left(\chi_{j}\right)_{f} \gamma_{f}\|\cdot\|_{f}^{\frac{n-2 j}{2}}\right)$. As all $B C\left(\chi_{j}\right)$ and $\gamma\|\cdot\|^{\frac{n-2 j}{2}}$ are algebraic Hecke characters, $\prod_{j=1}^{n-1} \mathbb{Q}\left(B C\left(\chi_{j}\right)_{f} \gamma_{f}\|\cdot\|_{f}^{\frac{n-2 j}{2}}\right)$ is a number field, whence so is $\mathbb{Q}\left(\Pi_{f}^{\prime}\right)$. The rest follows now from Clozel [5, Proposition 3.1 and p. 122].

Remark 2.6. The representation $\Pi_{f}^{\prime}$ is induced from the Hecke character $\beta:=\Pi_{1}^{\prime} \otimes \cdots \otimes$ $\Pi_{r}^{\prime}$ of the (Levi quotient $T^{\prime}\left(\mathbb{A}_{f}\right)$ of the) Borel subgroup $B^{\prime}\left(\mathbb{A}_{f}\right)$, and can thus be identified 
with a space of functions on $G^{\prime}\left(\mathbb{A}_{f}\right)$ :

$$
\Pi_{f}^{\prime}=\left\{\phi: G^{\prime}\left(\mathbb{A}_{f}\right) \rightarrow \mathbb{C} \mid \phi\left(b^{\prime} g^{\prime}\right)=\beta\left(b^{\prime}\right) \cdot \delta_{B^{\prime}}^{\frac{1}{2}}\left(b^{\prime}\right) \cdot \phi\left(g^{\prime}\right), b^{\prime} \in B^{\prime}\left(\mathbb{A}_{f}\right), g^{\prime} \in G^{\prime}\left(\mathbb{A}_{f}\right)\right\} .
$$

Here, $\delta_{B^{\prime}}$ is the modulus character of $B^{\prime}\left(\mathbb{A}_{f}\right)$. Define $\gamma^{+}(x)=\gamma(x) \cdot\|x\|^{\frac{1}{2}}$ if $n$ is odd, and $\gamma^{+}(x)=1$ if $n$ is even. For any $n,\left(\Pi_{j}^{\prime}\right)^{+}=B C\left(\chi_{j}\right) \cdot \gamma^{+}$is an algebraic Hecke character, defined over the number field $\mathbb{Q}\left(\left(\Pi_{j}^{\prime}\right)^{+}\right)$. On the other hand, the $j$ th component of $\beta \cdot \delta_{B^{\prime}}^{\frac{1}{2}}$ equals $B C\left(\chi_{j}\right) \cdot \gamma\|\cdot\|^{\frac{n-2 j}{2}}$, which is $\left(\Pi_{j}^{\prime}\right)^{+}$multiplied by an integral power of the norm character. Thus $\beta \cdot \delta_{B^{\prime}}^{\frac{1}{2}}$ takes values in a number field, say $\mathbb{Q}\left(\beta, \gamma^{+}\right)$. It follows that the subspace

$$
\begin{aligned}
& \Pi_{f}^{\prime}\left[\mathbb{Q}\left(\beta, \gamma^{+}\right)\right] \\
& \quad=\left\{\phi: G^{\prime}\left(\mathbb{A}_{f}\right) \rightarrow \mathbb{Q}\left(\beta, \gamma^{+}\right) \mid \phi\left(b^{\prime} g^{\prime}\right)=\beta\left(b^{\prime}\right) \cdot \delta_{B^{\prime}}^{\frac{1}{2}}\left(b^{\prime}\right) \cdot \phi\left(g^{\prime}\right), b^{\prime} \in B^{\prime}\left(\mathbb{A}_{f}\right), g^{\prime} \in G^{\prime}\left(\mathbb{A}_{f}\right)\right\}
\end{aligned}
$$

is a $G^{\prime}\left(\mathbb{A}_{f}\right)$-invariant $\mathbb{Q}\left(\beta, \gamma^{+}\right)$-rational structure on $\Pi_{f}^{\prime}$, and it is clear that the action of $\operatorname{Aut}(\mathbb{C})$ on $\Pi_{f}^{\prime}$ can be read directly in terms of the actions on the values of functions.

Next, let $\mathbf{t}_{\sigma, k}$ be the diagonal matrix $\operatorname{diag}\left(t_{\sigma}^{-(k-1)}, t_{\sigma}^{-(k-2)}, \ldots, 1\right) \in \mathrm{GL}_{k}\left(\mathbb{A}_{f}\right)$, as in $[40$, $3.2]$ or $[34,3.3]$. Here, $t_{\sigma}$ is an element of $\widehat{\mathcal{O}}^{*}$, assigned to $\sigma \in \operatorname{Aut}(\mathbb{C})$ by the map $\operatorname{Aut}(\mathbb{C}) \rightarrow$ $\operatorname{Gal}\left(\mathbb{Q}\left(\mu_{\infty}\right) / \mathbb{Q}\right) \rightarrow \widehat{\mathbb{Z}}^{*} \hookrightarrow \widehat{\mathcal{O}}^{*}$. Let $\xi_{f} \in \operatorname{Ind}_{N_{k}\left(\mathbb{A}_{f}\right)}^{\mathrm{GL}_{k}\left(\mathbb{A}_{f}\right)}\left[\phi_{f}\right]$ (unnormalized induction), $\phi$ an additive, continuous character $\mathcal{K} \backslash \mathbb{A}_{\mathcal{K}} \rightarrow \mathbb{C}^{\times}$. Then, ${ }^{\sigma} \xi_{f}\left(g_{f}\right):=\sigma\left(\xi\left(\mathbf{t}_{\sigma, k} g_{f}\right)\right)$ is again an element in $\operatorname{Ind}_{N_{k}\left(\mathbb{A}_{f}\right)}^{\mathrm{GL}_{k}\left(\mathbb{A}_{f}\right)}\left[\phi_{f}\right]$. The analogous definition applies locally at a finite place $w \in S(\mathcal{K})_{f}$.

Proposition 2.7. The map $\xi_{f} \mapsto{ }^{\sigma} \xi_{f}$ defines a $\sigma$-linear $G\left(\mathbb{A}_{f}\right)$-equivariant isomorphism from $W\left(\Pi_{f}\right)$ onto $W\left({ }^{\sigma} \Pi_{f}\right)$ as well as a $\sigma$-linear $G^{\prime}\left(\mathbb{A}_{f}\right)$-equivariant isomorphism from $W\left(\Pi_{f}^{\prime}\right)$ onto $W\left({ }^{\sigma} \Pi_{f}^{\prime}\right)$. For any finite extension $F$ of the rationality field in question, we have an $F$-structure on $W\left(\Pi_{f}\right)$ and $W\left(\Pi_{f}^{\prime}\right)$ by taking the $\operatorname{Aut}(\mathbb{C} / F)$-invariants.

Proof. For the cuspidal representation $\Pi$, this is [40, Lemma 3.2]. The same proof goes through for $\Pi^{\prime}$, using Jacquet, Piatetski-Shapiro, and Shalika's theorem (5.1.(i)) in $[27]$.

\subsection{Rational structures on cohomological Harish-Chandra modules}

The universal enveloping algebra $\mathcal{U}\left(\mathfrak{g}^{\prime}\right)$ has a canonical $\mathbb{Q}$-rational (vector space) structure determined by the $\mathbb{Q}$-reductive group $R_{\mathcal{K} / \mathbb{Q}}\left(G^{\prime}\right)$. Moreover, $K^{\prime}$ has a compatible $\mathbb{Q}$-rational structure, and it determines a $\mathbb{Q}$-rational Cartan decomposition $\mathfrak{g}^{\prime}=\mathfrak{k}^{\prime} \oplus \mathfrak{p}^{\prime}$. It thus makes sense to define the field of rationality of the $\left(\mathcal{U}\left(\mathfrak{g}^{\prime}\right), K^{\prime}\right)$-module $\Pi_{\infty}^{\prime}$ as the field $\mathbb{Q}\left(\Pi_{\infty}^{\prime}\right)$ fixed by the action of the subgroup of Aut $(\mathbb{C})$ that fixes $\Pi_{\infty}^{\prime}$ up to isomorphism. Now $\Pi_{\infty}^{\prime}$ is the $\mathcal{U}\left(\mathfrak{g}^{\prime}\right)$-module induced from a $\mathbb{Q}\left(E_{\lambda}\right)$-rational character of the Lie algebra of the Borel subgroup $B^{\prime}(\mathbb{C})$. In fact, it is given by non-normalized induction from an algebraic character of the torus $T^{\prime}(\mathbb{C})$, by the arguments already seen in 2.6. It follows 
that the subspace of $\Pi_{\infty}^{\prime}$ isotypic for the representation $E_{\lambda}$ of $K^{\prime}$ is defined over the number field $\mathbb{Q}\left(E_{\lambda}\right)$. Using the $\mathbb{Q}$-rational structure on $\mathfrak{p}^{\prime}$, we find that the relative Lie algebra cohomology complex $C^{\bullet}\left(\mathfrak{g}^{\prime}, K^{\prime}, \Pi_{\infty}^{\prime} \otimes E_{\lambda}\right)=\operatorname{Hom}_{K^{\prime}}\left(\Lambda^{\bullet}\left(\mathfrak{p}^{\prime}\right), \Pi_{\infty}^{\prime} \otimes E_{\lambda}\right)$ has a natural $\mathbb{Q}\left(E_{\lambda}\right)$-rational (vector space) structure. Hence, the same holds true for $\left(\mathfrak{g}^{\prime}, K^{\prime}\right)$-cohomology.

Although the cuspidal automorphic representation $\Pi$ is not globally induced, it follows from the classification of cohomological representations that the archimedean component $\Pi_{\infty}$ is again isomorphic to the non-normalized induction to $G(\mathbb{C})$ of an algebraic character of the maximal torus $T(\mathbb{C})$; see $\S 2.4$. We find again that $C^{\bullet}\left(\mathfrak{g}, K, \Pi_{\infty} \otimes E_{\mu}\right)=$ $\operatorname{Hom}_{K}\left(\Lambda^{\bullet}(\mathfrak{p}), \Pi_{\infty} \otimes E_{\mu}\right)$ - and so also the space of $(\mathfrak{g}, K)$-cohomology - has a natural $\mathbb{Q}\left(E_{\mu}\right)$-rational (vector space) structure.

In subsequent sections we will be working with the Whittaker models $W\left(\Pi_{\infty}\right)$ and $W\left(\Pi_{\infty}^{\prime}\right)$. It follows from the above discussion that their subspaces of $K$-finite (respectively, $K^{\prime}$-finite) vectors have rational models over the appropriate fields. Choices of complex isomorphisms $i_{\infty}: \Pi_{\infty} \stackrel{\sim}{\rightarrow} W\left(\Pi_{\infty}\right)$ and $i_{\infty}^{\prime}: \Pi_{\infty}^{\prime} \stackrel{\sim}{\rightarrow} W\left(\Pi_{\infty}^{\prime}\right)$ identify the rational models of the two sides, up to complex factors of proportionality that depend on the choices. In the proof of Proposition 5.7, the factor of proportionality is denoted $\Omega\left(\Pi_{\infty}^{\prime}\right)$; it is attached to the explicit choice of $i_{\infty}^{\prime}$ defined by the Whittaker integral. It is tempting to use the Whittaker integral to define $i_{\infty}$ as well. This would provide natural normalizations for all the archimedean constants that appear in our final formulas. However, other normalizations - for example, normalizations in terms of the archimedean local zeta integrals - may turn out to be more natural.

\section{Whittaker periods for the general linear group}

\subsection{Automorphic cohomology of locally symmetric spaces}

We define

$$
S_{n}:=G(\mathcal{K}) \backslash G\left(\mathbb{A}_{\mathcal{K}}\right) / K \quad \text { and } \quad S_{n-1}:=G^{\prime}(\mathcal{K}) \backslash G^{\prime}\left(\mathbb{A}_{\mathcal{K}}\right) / K^{\prime} .
$$

Moreover, we let

$$
\tilde{S}_{n-1}:=G^{\prime}(\mathcal{K}) \backslash G^{\prime}\left(\mathbb{A}_{\mathcal{K}}\right) / U(n-1) .
$$

Observe that $K \cong U(n) \mathbb{C}^{\times}=U(n) \mathbb{R}_{+}$and similarly $K^{\prime} \cong U(n-1) \mathbb{C}^{\times}=U(n-1) \mathbb{R}_{+}$, where $\mathbb{R}_{+}$denotes the topological connected component of the identity of the split component of the center of $G(\mathbb{C})$ and $G^{\prime}(\mathbb{C})$. In this way, the group $U(n-1)$ in the definition of $\tilde{S}_{n-1}$ is $K^{\prime}$ without the contribution of the center. Consider the map $\iota: G^{\prime} \hookrightarrow G$, realizing an element $g^{\prime} \in G^{\prime}$ as block diagonal matrix $\operatorname{diag}\left(g^{\prime}, 1\right) \in G$. If $K_{f}$ is an open compact subgroup of $G\left(\mathbb{A}_{f}\right), K_{f}^{\prime}:=\iota^{-1}\left(K_{f}\right)$ is open compact in $G^{\prime}\left(\mathbb{A}_{f}\right)$, and we define

$$
S_{n}\left(K_{f}\right):=G(\mathcal{K}) \backslash G\left(\mathbb{A}_{\mathcal{K}}\right) / K K_{f} \quad \text { and } \quad S_{n-1}\left(K_{f}^{\prime}\right):=G^{\prime}(\mathcal{K}) \backslash G^{\prime}\left(\mathbb{A}_{\mathcal{K}}\right) / K^{\prime} K_{f}^{\prime}
$$

and $\tilde{S}_{n-1}\left(K_{f}^{\prime}\right):=G^{\prime}(\mathcal{K}) \backslash G^{\prime}\left(\mathbb{A}_{\mathcal{K}}\right) / U(n-1) K_{f}^{\prime}$. Our finite-dimensional modules $E_{\mu}$ and $E_{\lambda}$ naturally define sheaves $\mathcal{E}_{\mu}$ on $S_{n}$ and $\mathcal{E}_{\lambda}$ on $S_{n-1}$, respectively, and we let

$$
H^{q}\left(S_{n}, \mathcal{E}_{\mu}\right) \text { and } H^{q}\left(S_{n-1}, \mathcal{E}_{\lambda}\right)
$$


be the corresponding cohomology spaces. They carry a $G\left(\mathbb{A}_{f}\right)$-module (respectively, a $G^{\prime}\left(\mathbb{A}_{f}\right)$-module) structure. With respect to this module structure, there are isomorphisms

$$
H^{q}\left(S_{n}, \mathcal{E}_{\mu}\right) \cong H^{q}\left(\mathfrak{g}, K, \mathcal{A}_{\mathcal{J}}(G) \otimes E_{\mu}\right)
$$

and

$$
H^{q}\left(S_{n-1}, \mathcal{E}_{\lambda}\right) \cong H^{q}\left(\mathfrak{g}^{\prime}, K^{\prime}, \mathcal{A}_{\mathcal{J}^{\prime}}\left(G^{\prime}\right) \otimes E_{\lambda}\right) .
$$

This needs some explanation. First, the space $\mathcal{A}_{\mathcal{J}}(G)$ (respectively, $\mathcal{A}_{\mathcal{J}^{\prime}}\left(G^{\prime}\right)$ ) denotes the space of all automorphic forms of $G\left(\mathbb{A}_{\mathcal{K}}\right)$ (respectively, $G^{\prime}\left(\mathbb{A}_{\mathcal{K}}\right)$ ) which are annihilated by some power of $\mathcal{J}$ (respectively, $\mathcal{J}^{\prime}$ ). Here, $\mathcal{J}$ (respectively, $\mathcal{J}^{\prime}$ ) is the ideal of the center of the universal enveloping algebra $\mathcal{U}\left(\mathfrak{g}_{\mathbb{C}}\right)$ (respectively, $\mathcal{U}\left(\mathfrak{g}_{\mathbb{C}}^{\prime}\right)$ ), which is annihilated by the contragredient representation of $E_{\mu}$ (respectively, $\left.E_{\lambda}\right)$. Now, since $U(n) \mathbb{C}^{\times}=U(n) \mathbb{R}_{+}$ and $U(n-1) \mathbb{C}^{\times}=U(n-1) \mathbb{R}_{+}$, the assertion follows from Franke, [9, Theorem 18].

From this, we obtain decompositions

$$
H^{q}\left(S_{n}, \mathcal{E}_{\mu}\right) \cong H_{\text {cusp }}^{q}\left(S_{n}, \mathcal{E}_{\mu}\right) \oplus H_{\text {Eis }}^{q}\left(S_{n}, \mathcal{E}_{\mu}\right)
$$

and

$$
H^{q}\left(S_{n-1}, \mathcal{E}_{\lambda}\right) \cong H_{\text {cusp }}^{q}\left(S_{n-1}, \mathcal{E}_{\lambda}\right) \oplus H_{\text {Eis }}^{q}\left(S_{n-1}, \mathcal{E}_{\lambda}\right)
$$

as follows: the space $\mathcal{A}_{\mathcal{J}}(G)$ may be decomposed along the associate classes $\{P\}$ of parabolic $\mathcal{K}$-subgroups $P=L N$ of $G$ and the cuspidal supports $\varphi_{P}$, i.e., associate classes of irreducible cuspidal automorphic representations $\tau=\tilde{\tau} e^{\left\langle d \Lambda, H_{P}(.)\right\rangle}$ of $L\left(\mathbb{A}_{\mathcal{K}}\right)$, with $\tilde{\tau}$ a unitary cuspidal automorphic representation and $\Lambda: \mathbb{R}_{+}^{\operatorname{rank}(P)} \rightarrow \mathbb{C}^{\times}$a Lie group character of the split component $\mathbb{R}_{+}^{\operatorname{rank}(P)}$ of $L(\mathbb{C})$. Indeed, if $\mathcal{A}_{\mathcal{J},\{P\}}(G)$ denotes the space of all automorphic forms in $\mathcal{A}_{\mathcal{J}}(G)$, which are negligible along every parabolic subgroup $Q \notin\{P\}$, and, moreover, if $\mathcal{A}_{\mathcal{J},\{P\}, \varphi_{P}}(G)$ denotes the subspace of $\mathcal{A}_{\mathcal{J},\{P\}}(G)$, which is generated as a $G(\mathbb{A})$-module by all possible holomorphic values or residues of all Eisenstein series attached to $\tilde{\tau}$, evaluated at the point $d \Lambda$, together with all their derivatives, then

$$
\mathcal{A}_{\mathcal{J}}(G)=\bigoplus_{\{P\}} \mathcal{A}_{\mathcal{J},\{P\}}(G)=\bigoplus_{\{P\}, \varphi_{P}} \mathcal{A}_{\mathcal{J},\{P\}, \varphi_{P}}(G)
$$

For a detailed description of this decomposition, we refer the reader to the original paper, by Franke and Schwermer [10, 1.1-1.4]. If we set

$$
\begin{gathered}
H_{c u s p}^{q}\left(S_{n}, \mathcal{E}_{\mu}\right):=H^{q}\left(\mathfrak{g}, K, \mathcal{A}_{\mathcal{J},\{G\}}(G) \otimes E_{\mu}\right) \\
H_{\text {Eis }}^{q}\left(S_{n}, \mathcal{E}_{\mu}\right):=\bigoplus_{\{P\} \neq\{G\}} H^{q}\left(\mathfrak{g}^{\prime}, K^{\prime}, \mathcal{A}_{\mathcal{J},\{P\}}(G) \otimes E_{\mu}\right),
\end{gathered}
$$

we obtain the above decomposition of cohomology for $G$. We remark that, because $\mathcal{A}_{\mathcal{J},\{G\}}(G)$ consists precisely of all cuspidal automorphic forms in $\mathcal{A}_{\mathcal{J}}(G), H_{\text {cusp }}^{q}\left(S_{n}, \mathcal{E}_{\mu}\right)$ is called the cuspidal cohomology of $G$ (with respect to $E_{\mu}$ ), and since $\mathcal{A}_{\mathcal{J},\{P\}}(G)$ is defined by means of Eisenstein series, supported in $\{P\}, H_{\text {Eis }}^{q}\left(S_{n}, \mathcal{E}_{\mu}\right)$ is called the Eisenstein cohomology of $G$ (with respect to $E_{\mu}$ ). Clearly, putting a prime everywhere gives the analogous result for the cohomology of $G^{\prime}$. 
Let $H_{c}^{q}\left(S_{n}, \mathcal{E}_{\mu}\right)$ (respectively, $\left.H_{c}^{q}\left(\tilde{S}_{n-1}, \mathcal{E}_{\lambda}\right)\right)$ be the cohomology with compact support. As cusp forms are rapidly decreasing, one has $H_{c u s p}^{q}\left(S_{n}, \mathcal{E}_{\mu}\right) \subseteq H_{c}^{q}\left(S_{n}, \mathcal{E}_{\mu}\right)$. Put $b_{k}:=$ $\frac{k(k-1)}{2}$. Then $b_{k}$ is the smallest degree in which a cohomological generic automorphic representation of $\mathrm{GL}_{k}\left(\mathbb{A}_{\mathcal{K}}\right)$ has non-zero cohomology. Note that this degree is independent of the given representation, as well as of the finite-dimensional coefficient module. Furthermore,

$$
H^{b_{n}}\left(\mathfrak{g}, K, W\left(\Pi_{\infty}\right) \otimes E_{\mu}\right) \cong \mathbb{C}
$$

and also

$$
H^{b_{n-1}}\left(\mathfrak{g}^{\prime}, K^{\prime}, W\left(\Pi_{\infty}^{\prime}\right) \otimes E_{\lambda}\right) \cong \mathbb{C}
$$

\subsection{A diagram}

Similar to the ideas in Mahnkopf [34], Raghuram [38, 39], and Grobner and Raghuram [16], we are going to consider the following diagram:

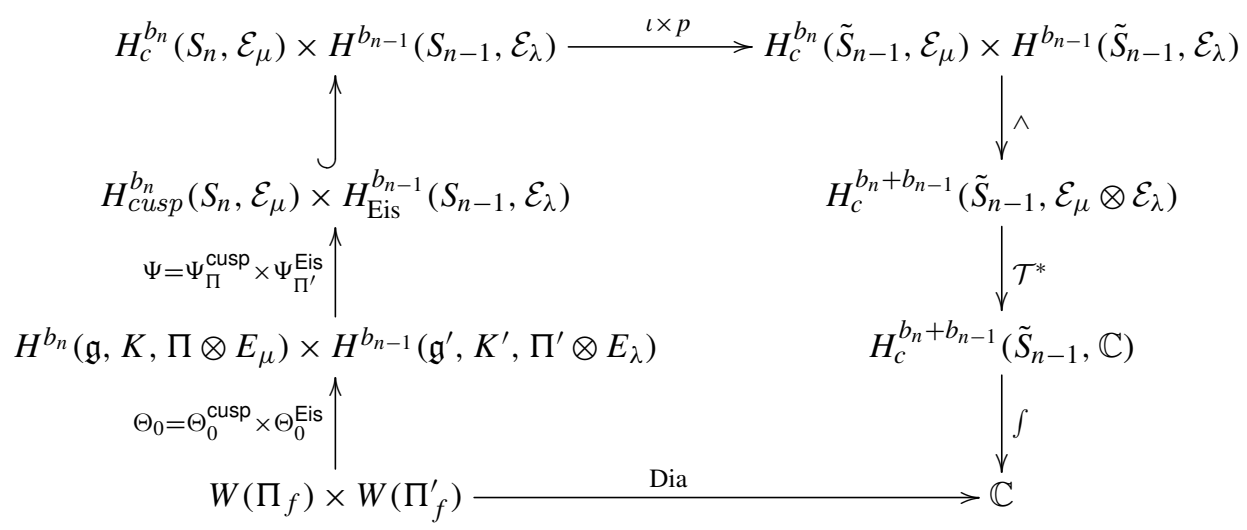

We are going to define the various maps, which appear in this diagram, in the next sections. Observe that Dia denotes the composition of all these maps.

\subsection{The map $\Theta_{0}$}

This section is in analogy with Mahnkopf [34], Raghuram and Shahidi, [40], Raghuram [38, 39] and Grobner and Raghuram [16].

As a first step, we choose and fix generators of the one-dimensional spaces $H^{b_{n}}\left(\mathfrak{g}, K, W\left(\Pi_{\infty}\right) \otimes E_{\mu}\right)$ and $H^{b_{n-1}}\left(\mathfrak{g}^{\prime}, K^{\prime}, W\left(\Pi_{\infty}^{\prime}\right) \otimes E_{\lambda}\right)$. They are of the form

$$
\left[\Pi_{\infty}\right]:=\sum_{\underline{i}=\left(i_{1}, \ldots, i_{b_{n}}\right)} \sum_{\alpha=1}^{\operatorname{dim} E_{\mu}} X_{\underline{i}}^{*} \otimes \xi_{\Pi_{\infty}, \underline{i}, \alpha} \otimes e_{\alpha}
$$

and

$$
\left[\Pi_{\infty}^{\prime}\right]:=\sum_{\underline{j}=\left(j_{1}, \ldots, j_{b_{n-1}}\right)} \sum_{\beta=1}^{\operatorname{dim} E_{\lambda}} X_{\underline{j}}^{*} \otimes \xi_{\Pi_{\infty}^{\prime}, \underline{j}, \beta} \otimes e_{\beta}^{\prime},
$$

where the following data has been fixed. 
(1) An ordered basis $\left\{X_{j}\right\}$ of $\mathfrak{g} / \mathfrak{k}$, which fixes the dual-basis $\left\{X_{j}^{*}\right\}$ for $(\mathfrak{g} / \mathfrak{k})^{*}$. By our concrete choice of $K, \mathfrak{k}$ is defined over $\mathbb{Q}$, whence we may assume that $\left\{X_{j}\right\}$ is a $\mathbb{Q}$-basis. For $\underline{i}=\left(i_{1}, \ldots, i_{b_{n}}\right)$, let $X_{\underline{i}}^{*}=X_{i_{1}}^{*} \wedge \cdots \wedge X_{i_{b_{n}}}^{*} \in \bigwedge^{b_{n}}(\mathfrak{g} / \mathfrak{k})^{*}$.

(2) Elements $e_{1}, \ldots, e_{\operatorname{dim} E_{\mu}}$, which form a $\mathbb{Q}\left(E_{\mu}\right)$-basis of $E_{\mu}$.

(3) To each $\underline{i}$ and $\alpha, \xi_{\Pi_{\infty}, \underline{i}, \alpha} \in W\left(\Pi_{\infty}\right)$.

In addition, we have the following.

(1) An ordered basis $\left\{X_{j}^{\prime}\right\}$ of $\mathfrak{g}^{\prime} / \mathfrak{k}^{\prime}$, which fixes the dual-basis $\left\{X_{j}^{*}\right\}$ for $\left(\mathfrak{g}^{\prime} / \mathfrak{k}^{\prime}\right)^{*}$. Similar to the case above, by our concrete choice of $K^{\prime}, \mathfrak{k}^{\prime}$ is defined over $\mathbb{Q}$, whence we may assume that $\left\{X_{j}^{\prime}\right\}$ is a $\mathbb{Q}$-basis. For $\underline{j}=\left(j_{1}, \ldots, j_{b_{n-1}}\right)$, let $X_{\underline{j}}^{\prime *}=X_{j_{1}}^{*} \wedge \cdots \wedge X_{j_{b_{n-1}}}^{\prime *} \in$

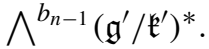

(2) Elements $e_{1}^{\prime}, \ldots, e_{\operatorname{dim} E_{\lambda}}^{\prime}$, which form a $\mathbb{Q}\left(E_{\lambda}\right)$-basis of $E_{\lambda}$.

(3) To each $\underline{j}$ and $\beta, \xi_{\Pi_{\infty}^{\prime}, \underline{j}, \beta} \in W\left(\Pi_{\infty}^{\prime}\right)$.

We may assume that the bases are compatible, in the sense that $\left\{X_{j}\right\}$ extends $\left\{X_{j}^{\prime}\right\}$ (along the embedding $\mathfrak{g}^{\prime} / \mathfrak{k}^{\prime} \hookrightarrow \mathfrak{g} / \mathfrak{k}$ defined by $\iota$ ) and that the non-compact part of the center of $\mathfrak{g}^{\prime}$ is spanned by $X_{r}, r=\operatorname{dim}_{\mathbb{R}} \tilde{S}_{n-1}$. The choice of the generators [ $\Pi_{\infty}$ ] and [ $\Pi_{\infty}^{\prime}$ ] fixes generators [ $\left.{ }^{\sigma} \Pi_{\infty}\right]$ and $\left[{ }^{\sigma} \Pi_{\infty}^{\prime}\right.$ ] of the one-dimensional spaces $H^{b_{n}}\left(\mathfrak{g}^{\prime}, K^{\prime}, W\left({ }^{\sigma} \Pi_{\infty}\right) \otimes\right.$ $\left.{ }^{\sigma} E_{\mu}\right)$ and $H^{b_{n-1}}\left(\mathfrak{g}^{\prime}, K^{\prime}, W\left({ }^{\sigma} \Pi_{\infty}^{\prime}\right) \otimes{ }^{\sigma} E_{\lambda}\right)$ for all $\sigma \in \operatorname{Aut}(\mathbb{C})$ as well as isomorphisms $\Theta^{\text {cusp }}$ and $\Theta^{\text {Eis }}$ :

$$
\begin{aligned}
W\left(\Pi_{f}\right) & \stackrel{\sim}{\rightarrow} W\left(\Pi_{f}\right) \otimes H^{b_{n}}\left(\mathfrak{g}, K, W\left(\Pi_{\infty}\right) \otimes E_{\mu}\right) \\
& \stackrel{\sim}{\rightarrow} H^{b_{n}}\left(\mathfrak{g}, K, W(\Pi) \otimes E_{\mu}\right) \\
& \stackrel{\sim}{\rightarrow} H^{b_{n}}\left(\mathfrak{g}, K, \Pi \otimes E_{\mu}\right)
\end{aligned}
$$

and

$$
\begin{aligned}
W\left(\Pi_{f}^{\prime}\right) & \stackrel{\sim}{\rightarrow} W\left(\Pi_{f}^{\prime}\right) \otimes H^{b_{n-1}}\left(\mathfrak{g}^{\prime}, K^{\prime}, W\left(\Pi_{\infty}^{\prime}\right) \otimes E_{\lambda}\right) \\
& \stackrel{\sim}{\rightarrow} H^{b_{n-1}}\left(\mathfrak{g}^{\prime}, K^{\prime}, W\left(\Pi^{\prime}\right) \otimes E_{\lambda}\right) \\
& \stackrel{\sim}{\rightarrow} H^{b_{n-1}}\left(\mathfrak{g}^{\prime}, K^{\prime}, \Pi^{\prime} \otimes E_{\lambda}\right),
\end{aligned}
$$

where the last map in the respective diagram denotes the inverse of the corresponding Fourier coefficient. Next, recall from Proposition 2.5 that the $G\left(\mathbb{A}_{f}\right)$-module $H^{b_{n}}\left(\mathfrak{g}, K, \Pi \otimes E_{\mu}\right)$ is defined over $\mathbb{Q}\left(\Pi_{f}\right)$ and that the $G^{\prime}\left(\mathbb{A}_{f}\right)$-module $H^{b_{n-1}}\left(\mathfrak{g}^{\prime}, K^{\prime}, \Pi^{\prime} \otimes\right.$ $\left.E_{\lambda}\right)$ is defined over $\mathbb{Q}\left(\Pi_{f}^{\prime}\right)$. Both structures are unique up to multiplication by non-zero complex numbers. This leads us to the following.

Proposition 3.1 (The Whittaker periods). There are non-zero complex numbers $p(\Pi)=$ $p\left(\Pi_{f},\left[\Pi_{\infty}\right]\right)$ and $p\left(\Pi^{\prime}\right)=p\left(\Pi_{f}^{\prime},\left[\Pi_{\infty}^{\prime}\right]\right)$, such that the normalized maps

$$
\Theta_{0}^{\text {cusp }}:=p(\Pi)^{-1} \cdot \Theta^{\text {cusp }} \quad \text { and } \quad \Theta_{0}^{\text {Eis }}:=p\left(\Pi^{\prime}\right)^{-1} \cdot \Theta^{\text {Eis }}
$$


are $\operatorname{Aut}(\mathbb{C})$-equivariant, i.e.,

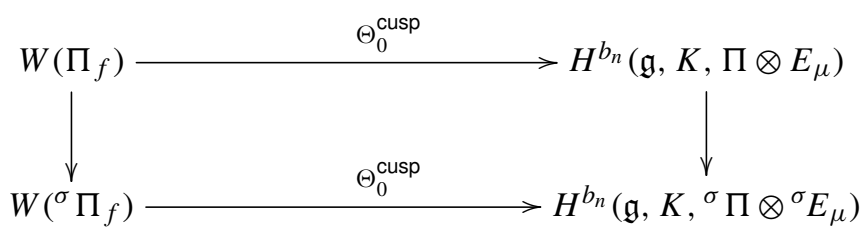

and

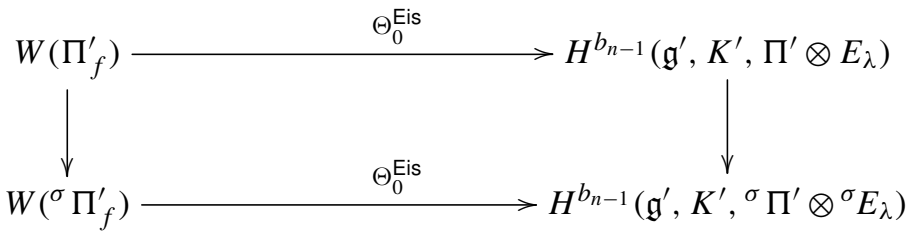

commute. The complex number $p(\Pi)$ (respectively, $\left.p\left(\Pi^{\prime}\right)\right)$ is well defined only up to multiplication by non-zero elements of the number field $\mathbb{Q}\left(\Pi_{f}\right)$ (respectively, $\mathbb{Q}\left(\Pi_{f}^{\prime}\right)$ ).

Proof. For $\Pi$, this is shown in [40, Definition/Proposition 3.3]. In order to obtain the result for $\Pi^{\prime}$, we observe that the proof of [40, Definition/Proposition 3.3] (respectively, [16, Definition/Proposition 4.2.1]) goes over word for word, keeping in mind our Proposition 2.7 and Jacquet, Piatetski-Shapiro, and Shalika's Theorem (5.1.(i)) in $[27]$.

Finally, we set

$$
\Theta_{0}:=\Theta_{0}^{\text {cusp }} \times \Theta_{0}^{\text {Eis }} .
$$

\subsection{The map $\Psi=\Psi_{\Pi}^{\text {cusp }} \times \Psi_{\Pi^{\prime}}^{\text {Eis }}$}

It is well known and follows from our résumé in $\S 3.1$ together with Multiplicity One that

$$
H^{b_{n}}\left(\mathfrak{g}, K, \Pi \otimes E_{\mu}\right)=H^{b_{n}}\left(\mathfrak{g}, K, \mathcal{A}_{\mathcal{J},\{G\}, \varphi_{\Pi}} \otimes E_{\mu}\right),
$$

where $\varphi_{\Pi}$ is a singleton, represented by the cuspidal automorphic representation $\Pi$. Hence, there is a natural embedding

$$
\Psi_{\Pi}^{\text {cusp }}: H^{b_{n}}\left(\mathfrak{g}, K, \Pi \otimes E_{\mu}\right) \hookrightarrow H_{c u s p}^{b_{n}}\left(S_{n}, \mathcal{E}_{\mu}\right) .
$$

It is the purpose of this section to construct an embedding

$$
\Psi_{\Pi^{\prime}}^{\text {Eis }}: H^{b_{n-1}}\left(\mathfrak{g}^{\prime}, K^{\prime}, \Pi^{\prime} \otimes E_{\lambda}\right) \hookrightarrow H_{E i s}^{b_{n-1}}\left(S_{n-1}, \mathcal{E}_{\lambda}\right),
$$

as well. This is more delicate. First, as a short remark, let us point out that - in contrast to the case of cuspidal cohomology - this is also a question of degrees of cohomology. For a non-trivial $G^{\prime}\left(\mathbb{A}_{f}\right)$-morphism $H^{b_{n-1}}\left(\mathfrak{g}^{\prime}, K^{\prime}, \Pi^{\prime} \otimes E_{\lambda}\right) \rightarrow H_{\text {Eis }}^{b_{n-1}}\left(S_{n-1}, \mathcal{E}_{\lambda}\right)$ to exist, it is necessary that

$$
q_{\max }:=\min _{\substack{P^{\prime}=a \max \\ \text { parabolic } / \mathcal{K}}}\left(\frac{1}{2} \operatorname{dim}_{\mathbb{R}} N_{P^{\prime}}(\mathbb{C})\right) \leqslant b_{n-1} .
$$

This follows from Grobner [13, Theorem 18], since $\Pi^{\prime}$ is not square integrable. Since, for any maximal parabolic subgroup $P^{\prime}$ of $G^{\prime}$, the unipotent radical $N_{P^{\prime}}$ is of 
dimension $\operatorname{dim}_{\mathbb{C}} N_{P^{\prime}}(\mathbb{C})=m_{1} \cdot m_{2}$, for some non-trivial partition $m_{1}+m_{2}=n-1$, we have without loss of generality that $m_{1} \leqslant \frac{n-1}{2}$ and $m_{2} \leqslant n-2$. Hence,

$$
q_{\max } \leqslant \frac{1}{2} \operatorname{dim}_{\mathbb{R}} N_{P^{\prime}}(\mathbb{C})=m_{1} \cdot m_{2} \leqslant \frac{(n-1)(n-2)}{2}=b_{n-1},
$$

and therefore the above condition is always satisfied. We now construct such a non-trivial map and show that is an injection.

Since $b_{n-1}$ is the minimal degree, in which $\Pi_{\infty}^{\prime} \cong \operatorname{Ind}_{B^{\prime}(\mathbb{C})}^{G^{\prime}(\mathbb{C})}\left[z_{1}^{k_{1}+c} \bar{z}_{1}^{-k_{1}-c} \otimes \cdots \otimes\right.$ $z_{n-1}^{k_{n-1}+c} \bar{z}_{n-1}^{-k_{n-1}-c}$ ] has non-vanishing $\left(\mathfrak{g}^{\prime}, K^{\prime}\right)$-cohomology with respect to $E_{\lambda}$, there is an isomorphism of one-dimensional $\mathbb{C}$-vector-spaces:

$$
\begin{aligned}
& H^{b_{n-1}}\left(\mathfrak{g}^{\prime}, K^{\prime}, \operatorname{Ind}_{B^{\prime}(\mathbb{C})}^{G^{\prime}(\mathbb{C})}\left[z_{1}^{k_{1}+c} \bar{z}_{1}^{-k_{1}-c} \otimes \cdots \otimes z_{n-1}^{k_{n-1}+c} \bar{z}_{n-1}^{-k_{n-1}-c}\right] \otimes E_{\lambda}\right) \\
& \quad \stackrel{\sim}{\rightarrow} H^{b_{n-1}}\left(\mathfrak{g}^{\prime}, K^{\prime}, \operatorname{Ind}_{B^{\prime}(\mathbb{C})}^{G^{\prime}(\mathbb{C})}\left[z_{1}^{k_{1}+c} \bar{z}_{1}^{-k_{1}-c} \otimes \cdots \otimes z_{n-1}^{k_{n-1}+c} \bar{z}_{n-1}^{-k_{n-1}-c} \otimes S\left(\check{\mathfrak{a}}_{B^{\prime}, \mathbb{C}}^{G^{\prime}}\right)\right] \otimes E_{\lambda}\right),
\end{aligned}
$$

where $S\left(\check{\mathfrak{a}}_{B^{\prime}, \mathbb{C}}^{G^{\prime}}\right)$ is the symmetric algebra of the orthogonal complement $\check{\mathfrak{a}}_{B^{\prime}, \mathbb{C}}^{G^{\prime}}$ of $\check{\mathfrak{a}}_{G^{\prime}, \mathbb{C}}=X^{*}\left(\dot{G}^{\prime}\right) \otimes_{\mathbb{Z}} \mathbb{C}$ in $\check{\mathfrak{a}}_{B^{\prime}, \mathbb{C}}=X^{*}\left(T^{\prime}\right) \otimes_{\mathbb{Z}} \mathbb{C}$. Hence, if we write $\tilde{\tau}:=B C\left(\chi_{1}\right) \gamma \otimes \cdots \otimes$ $B C\left(\chi_{n-1}\right) \gamma$, then

$$
H^{b_{n-1}}\left(\mathfrak{g}^{\prime}, K^{\prime}, \Pi^{\prime} \otimes E_{\lambda}\right) \cong H^{b_{n-1}}\left(\mathfrak{g}^{\prime}, K^{\prime}, \operatorname{Ind}_{B^{\prime}\left(\mathbb{A}_{\mathcal{K}}\right)}^{G^{\prime}\left(\mathbb{K}_{\mathcal{K}}\right)}\left[\tilde{\tau} \otimes S\left(\check{\mathfrak{a}}_{B^{\prime}, \mathbb{C}}^{G^{\prime}}\right)\right] \otimes E_{\lambda}\right) .
$$

The algebra $\mathfrak{a}_{B^{\prime}, \mathbb{C}}^{G^{\prime}}$ operates trivially on $\tilde{\tau}$. Hence, one may check that $\left(B^{\prime}, \tilde{\tau}, 0,0\right)$ is one of the quadruples, constructed in Grobner $[13,3.3]$. Let $\varphi_{B^{\prime}}$ be the associate class of unitary cuspidal automorphic representations of $T^{\prime}\left(\mathbb{A}_{\mathcal{K}}\right)$, represented by $\tilde{\tau}$, so the summand $\mathcal{A}_{\mathcal{J}^{\prime},\left\{B^{\prime}\right\}, \varphi_{B^{\prime}}}\left(G^{\prime}\right)$ of the space of automorphic forms $\mathcal{A}_{\mathcal{J}^{\prime}}\left(G^{\prime}\right)$, cf. $\S 3.1$, is well defined. Interpreting the elements of the symmetric algebra as differential operators $\frac{\partial^{m}}{\partial \Lambda^{m}}$, we obtain an intertwining operator

$$
\begin{gathered}
\operatorname{Eis}_{\Pi^{\prime}}: \operatorname{Ind}_{B^{\prime}\left(\mathbb{A}_{\mathcal{K}}\right)}^{G^{\prime}\left(\mathbb{A}_{\mathcal{L}}\right)}\left[\tilde{\tau} \otimes S\left(\check{\mathfrak{a}}_{B^{\prime}, \mathbb{C}}^{G^{\prime}}\right)\right] \rightarrow \mathcal{A}_{\mathcal{J}^{\prime},\left\{B^{\prime}\right\}, \varphi_{B^{\prime}}}\left(G^{\prime}\right) \\
\left.f \otimes \frac{\partial^{m}}{\partial \Lambda^{m}} \mapsto \frac{\partial^{m}}{\partial \Lambda^{m}}(E(f, \Lambda))\right|_{\Lambda=0},
\end{gathered}
$$

where $E(f, \Lambda)$ is the Eisenstein series attached to a $K^{\prime}$-finite section $f \in \operatorname{Ind}_{B^{\prime}\left(\mathbb{A}_{\mathcal{K}}\right)}^{G^{\prime}\left(\mathbb{\tau}_{\mathcal{C}}\right] \text { at }}$ $\Lambda \in \mathfrak{a}_{B^{\prime}, \mathbb{C}}^{G^{\prime}}$. Observe that this intertwining operator is well defined, since all Eisenstein series are holomorphic at $\Lambda=0$.

Proposition 3.3. The $G^{\prime}\left(\mathbb{A}_{f}\right)$-homomorphism

$$
\Psi_{\Pi^{\prime}}: H^{b_{n-1}}\left(\mathfrak{g}^{\prime}, K^{\prime}, \operatorname{Ind}_{B^{\prime}\left(\mathbb{A}_{\mathcal{K}}\right)}^{G^{\prime}\left(\mathbb{K}_{\mathcal{C}}\right)}\left[\tilde{\tau} \otimes S\left(\check{\mathfrak{a}}_{B^{\prime}, \mathbb{C}}^{G^{\prime}}\right)\right] \otimes E_{\lambda}\right) \rightarrow H^{b_{n-1}}\left(\mathfrak{g}^{\prime}, K^{\prime}, \mathcal{A}_{\mathcal{J}^{\prime},\left\{B^{\prime}\right\}, \varphi_{B^{\prime}}}\left(G^{\prime}\right) \otimes E_{\lambda}\right)
$$

induced from Eis $_{\Pi^{\prime}}$ is an injection.

Proof. As all Eisenstein series $E(f, \Lambda)$ attached to a $K^{\prime}$-finite section $f \in \operatorname{Ind}_{B^{\prime}\left(\mathbb{A}_{\mathcal{K}}\right)}^{G^{\prime}(\tilde{\tau}] \text { are }}$ holomorphic at $\Lambda=0, \Psi_{\Pi^{\prime}}$ is injective by Schwermer [43, Satz 4.11] or Li and Schwermer [32, Theorem 3.3]. 
As a consequence of our discussion in $\S 3.1$, we obtain an injection of $G^{\prime}\left(\mathbb{A}_{f}\right)$-modules

$$
\Psi_{\Pi^{\prime}}^{\text {Eis }}: H^{b_{n-1}}\left(\mathfrak{g}^{\prime}, K^{\prime}, \Pi^{\prime} \otimes E_{\lambda}\right)=H^{b_{n-1}}\left(\mathfrak{g}^{\prime}, K^{\prime}, \Pi_{\infty}^{\prime} \otimes E_{\lambda}\right) \otimes \Pi_{f}^{\prime} \hookrightarrow H_{\text {Eis }}^{b_{n-1}}\left(S_{n-1}, \mathcal{E}_{\lambda}\right) .
$$

With respect to the $\mathbb{Q}\left(E_{\lambda}\right)$-rational structure on relative Lie algebra cohomology defined in 2.7, this defines a $\mathbb{Q}\left(\Pi_{f}^{\prime}\right)$-rational injection

$$
\Pi_{f}^{\prime}=\operatorname{Ind}_{B^{\prime}\left(\mathbb{A}_{f}\right)}^{G^{\prime}\left(\mathbb{A}_{f}\right)}\left[\tilde{\tau}_{f}\right] \hookrightarrow H_{\mathrm{Eis}}^{b_{n-1}}\left(S_{n-1}, \mathcal{E}_{\lambda}\right),
$$

where, for any field $L \supseteq \mathbb{Q}\left(\Pi_{f}^{\prime}\right)$, the $L$-rational vectors on the left-hand side are just the $L$-valued functions on $G^{\prime}\left(\mathbb{A}_{f}\right)$ that transform on the left under the character $\delta_{B^{\prime}, f}^{\frac{1}{2}} \cdot \tilde{\tau}_{f}$.

\subsection{The map $\iota \times \phi$}

Recall the map $\iota: G^{\prime} \hookrightarrow G$, realizing an element $g^{\prime} \in G^{\prime}$ as block diagonal matrix $\operatorname{diag}\left(g^{\prime}, 1\right) \in G$.

Lemma 3.4. The map $\iota: \tilde{S}_{n-1}\left(K_{f}^{\prime}\right) \rightarrow S_{n}\left(K_{f}\right)$ is proper.

Proof. For the general linear group over $\mathbb{Q}$, this is stated in Mahnkopf [34, p. 615], however, without proof. For sake of completeness, we sketch an argument here. Let

$$
S_{n-1, n}\left(K_{f}\right):=M(F) \backslash M(\mathbb{A}) /(K \cap M(\mathbb{C}))\left(K_{f} \cap M\left(\mathbb{A}_{f}\right)\right),
$$

where $M \cong \mathrm{GL}_{n-1} \times \mathrm{GL}_{1}$, viewed as block diagonal matrices. The map $\iota$ factors as

$$
\tilde{S}_{n-1}\left(K_{f}^{\prime}\right) \stackrel{j}{\longrightarrow} S_{n-1, n}\left(K_{f}\right) \stackrel{u}{\longrightarrow} S_{n}\left(K_{f}\right) .
$$

Clearly, $j$ is proper. Hence, it suffices to show that $u$ is proper. This follows from [1, Lemma 2.7].

As a consequence, we obtain a map in cohomology with compact support:

$$
\iota^{q}: H_{c}^{q}\left(S_{n}\left(K_{f}\right), \mathcal{E}_{\mu}\right) \rightarrow H_{c}^{q}\left(\tilde{S}_{n-1}\left(K_{f}^{\prime}\right), \mathcal{E}_{\mu}\right) .
$$

Similarly, the projection $p: \tilde{S}_{n-1}\left(K_{f}^{\prime}\right) \rightarrow S_{n-1}\left(K_{f}^{\prime}\right)$ induces a map

$$
p^{q}: H^{q}\left(S_{n-1}\left(K_{f}^{\prime}\right), \mathcal{E}_{\lambda}\right) \rightarrow H^{q}\left(\tilde{S}_{n-1}\left(K_{f}^{\prime}\right), \mathcal{E}_{\lambda}\right) .
$$

In our diagram, we let $\iota \times p$ be the direct limit (over all open compact subgroups $K_{f}$ of $\left.G\left(\mathbb{A}_{f}\right)\right)$ of the maps $\iota^{b_{n}} \times p^{b_{n-1}}$.

\subsection{Critical points}

Recall the definition of a point $s=\frac{1}{2}+m, m \in \mathbb{Z}$ being critical for $L\left(s, \Pi \times \Pi^{\prime}\right)$ from Deligne, [8, Prop.-Déf. 2.3]. We let

$$
\operatorname{Crit}\left(\Pi \times \Pi^{\prime}\right) \subset \frac{1}{2}+\mathbb{Z}
$$

be the set of critical points of $L\left(s, \Pi \times \Pi^{\prime}\right)$. 
Lemma 3.5. If $s=\frac{1}{2} \pm m, \quad m \geqslant 0$, is a critical point of $L\left(s, \Pi \times \Pi^{\prime}\right)$, then $\operatorname{Hom}_{H^{\prime}(\mathbb{C})}\left(E_{\mu-m-\mathrm{m}}^{\mathrm{unt}} \otimes E_{\lambda}^{\mathrm{unt}}, \mathbb{C}\right) \neq 0$ and $\operatorname{Hom}_{H^{\prime}(\mathbb{C})}\left(E_{\mu^{\mathrm{v}}-m-\mathrm{m}}^{\mathrm{unt}} \otimes E_{\lambda^{\mathrm{v}}}^{\mathrm{unt}}, \mathbb{C}\right) \neq 0$. The set of critical points is $\operatorname{Aut}(\mathbb{C})$-invariant, i.e., $\operatorname{Crit}\left(\Pi \times \Pi^{\prime}\right)=\operatorname{Crit}\left({ }^{\sigma} \Pi \times{ }^{\sigma} \Pi^{\prime}\right)$.

Proof. A proof of these facts will be given in $\S 4$ in the motivic context. See in particular the proof of Lemma 4.7.

\subsection{The map $\mathcal{T}^{*}$}

Let $s=\frac{1}{2}+m \in \operatorname{Crit}\left(\Pi \times \Pi^{\prime}\right), m \geqslant 0$. Hence, by Lemma 3.5, we obtain a non-trivial map

$$
\mathcal{T}^{(m)} \in \operatorname{Hom}_{G^{\prime}(\mathbb{C})}\left(E_{\mu-m} \otimes E_{\lambda}, \mathbb{C}\right),
$$

which we will fix in a compatible way (i.e., $\mathcal{T}^{(m)}$ for $\Pi \otimes \Pi^{\prime}$ shall be identical to $\mathcal{T}^{(0)}$ for $\left.\left(\Pi\|\cdot\|^{m}\right) \otimes \Pi^{\prime}\right)$. Again by Lemma 3.5, it factors as $\mathcal{T}^{(m)}=\mathcal{T}_{\imath}^{(m)} \otimes \mathcal{T}_{\bar{\iota}}^{(m)}$, and if $\sigma \in$ Aut $(\mathbb{C})$, then we define ${ }^{\sigma}\left(\mathcal{T}^{(m)}\right)$ in the obvious way, i.e., ${ }^{\sigma}\left(\mathcal{T}^{(m)}\right)=\mathcal{T}_{\sigma \circ l}^{(m)} \otimes \mathcal{T}_{\overline{\sigma o l}}^{(m)}$. Finally, if $s=\frac{1}{2} \in \operatorname{Crit}\left(\Pi \times \Pi^{\prime}\right)$, we let $\mathcal{T}=\mathcal{T}^{(0)}$ and obtain a morphism in cohomology

$$
\mathcal{T}^{*}: H_{c}^{b_{n}+b_{n-1}}\left(\tilde{S}_{n-1}, \mathcal{E}_{\mu} \otimes \mathcal{E}_{\lambda}\right) \rightarrow H_{c}^{b_{n}+b_{n-1}}\left(\tilde{S}_{n-1}, \mathbb{C}\right),
$$

as in our diagram.

\subsection{Poincaré duality}

It remains to define the last map, denoted ' $\int$ ' in our diagram. Therefore, observe that

$$
b_{n}+b_{n-1}=\operatorname{dim}_{\mathbb{R}} \tilde{S}_{n-1} .
$$

Whence, we may use Poincaré duality, in order to obtain a surjective map $\int$ : $H_{c}^{b_{n}+b_{n-1}}\left(\tilde{S}_{n-1}, \mathbb{C}\right) \rightarrow \mathbb{C}$. To that end, we fix a $\mathbb{Q}$-valued Haar measure $d g_{f}$ on $G^{\prime}\left(\mathbb{A}_{f}\right)$ as follows. For a non-archimedean place $w \in S(\mathcal{K})_{f}$, we let $d g_{w}$ be the unique Haar measure on $G^{\prime}\left(\mathcal{K}_{w}\right)$, which gives $G^{\prime}\left(\mathcal{O}_{w}\right)$ volume 1 , and let $d g_{f}$ be the product of these local measures. With this choice of a measure, for any open compact subgroup $K_{f}^{\prime}$ of $G^{\prime}\left(\mathbb{A}_{f}\right)$, the volume $\operatorname{vol}\left(K_{f}^{\prime}\right)$ is a rational number. The set of connected components of the corresponding orbifold $\tilde{S}_{n-1}\left(K_{f}^{\prime}\right)$ is parameterized by the finite set $\mathcal{X}:=\mathbb{A}_{f}^{\times} / \mathcal{K}^{\times} \operatorname{det}\left(K_{f}^{\prime}\right)$. We assume that we have fixed orientations on the various connected components $\tilde{S}_{n-1}\left(K_{f}^{\prime}\right)_{x}, x \in \mathcal{X}$, given by the orientation on $G^{\prime}(\mathbb{C}) / U(n-1)$, which is defined by the ordered basis $\left\{X_{1}, \ldots, X_{r}\right\}$ of its tangent space at the identity; cf. $\S 3.3$. Hence, the de Rham isomorphism, which is given by integrating over $\tilde{S}_{n-1}\left(K_{f}^{\prime}\right)_{x}$, gives rise to a surjective map on $\tilde{S}_{n-1}\left(K_{f}^{\prime}\right)$,

$$
\int_{\tilde{S}_{n-1}\left(K_{f}^{\prime}\right)}=\sum_{x \in \mathcal{X}} \int_{\tilde{S}_{n-1}\left(K_{f}^{\prime}\right)_{x}} .
$$

Let $\int_{\tilde{S}_{n-1}}$ stand for the direct limit (over all open compact subgroups $K_{f}^{\prime}$ of $G^{\prime}\left(\mathbb{A}_{f}\right)$ ) of the normalized maps $\operatorname{vol}\left(K_{f}^{\prime}\right) \cdot \int_{\tilde{S}_{n-1}\left(K_{f}^{\prime}\right)}$, and observe that the trivial character $\mathbf{1}$ of $G^{\prime}\left(\mathbb{A}_{\mathcal{K}}\right)$ defines a non-trivial cohomology class in $H^{0}\left(\tilde{S}_{n-1}, \mathbb{C}\right)$. Using this class, the 
Poincaré-Duality pairing of $H_{c}^{b_{n}+b_{n-1}}\left(\tilde{S}_{n-1}, \mathbb{C}\right)$ and $H^{0}\left(\tilde{S}_{n-1}, \mathbb{C}\right)$ finally gives rise to a surjection

$$
\begin{aligned}
H_{c}^{b_{n}+b_{n-1}}\left(\tilde{S}_{n-1}, \mathbb{C}\right) & \longrightarrow \\
\theta & \longmapsto \int(\theta):=\int_{\tilde{S}_{n-1}} \theta .
\end{aligned}
$$

\subsection{A non-archimedean, particular vector}

We will now choose a special vector in the product of Whittaker models $W\left(\Pi_{f}\right) \times W\left(\Pi_{f}^{\prime}\right)$, which has the property that it transforms nicely, when plugged into our diagram. This vector will be fixed as in Raghuram [39, 3.1.4], which is itself inspired by Mahnkopf $[34,2.1 .1]$.

First, we remark that, for $w \in S(\mathcal{K})_{f}, \Pi_{w}^{\prime}$ is tempered. Hence, Jacquet and Shalika's [29] Proposition (3.2) still holds for $\Pi_{w}^{\prime}$. Therefore, any non-zero Whittaker functional $\xi_{w}^{\prime} \in$ $W\left(\Pi_{w}^{\prime}\right)$ is non-vanishing on $T^{\prime}\left(\mathcal{K}_{w}\right)^{+}=\left\{t \in T^{\prime}\left(\mathcal{K}_{w}\right) \mid t_{i} t_{i+1}^{-1} \in \mathcal{O}_{w}, t_{n-1, n-1}=1\right\}$. We let $K\left(m_{w}\right)$ (respectively, $\left.K^{\prime}\left(m_{w}^{\prime}\right)\right)$ be the mirahoric subgroup of $G\left(\mathcal{K}_{w}\right)$ (respectively, $G^{\prime}\left(\mathcal{K}_{w}\right)$ ) of level $m_{w}$ (respectively, $m_{w}^{\prime}$ ). This is the subgroup of $G\left(\mathcal{O}_{w}\right)$ (respectively, $G^{\prime}\left(\mathcal{O}_{w}\right)$ ) consisting of those matrices whose last row is congruent to $(0, \ldots, 0, \star)$ modulo $\wp_{w}^{m_{w}}$, where $\wp_{w}$ is the unique maximal ideal in $\mathcal{O}_{w}$. Suppose from now on that $m_{w}$ (respectively, $m_{w}^{\prime}$ ) is the conductor of $\Pi_{w}$ (respectively, $\Pi_{w}^{\prime}$ ). Then, by Jacquet, Piatetski-Shapiro, and Shalika's Theorem (5.1) [27], the space of Whittaker vectors transforming by the central character $\omega_{\Pi_{w}}$ of $\Pi_{w}$ (respectively, $\omega_{\Pi_{w}^{\prime}}$ of $\Pi_{w}^{\prime}$ ) under the action of the mirahoric subgroup is one dimensional, and its elements are usually called new vectors.

Now, for $\Pi_{w}^{\prime}$, fix an element $t_{\Pi_{w}^{\prime}} \in T^{\prime}\left(\mathcal{K}_{w}\right)^{+}$on which the non-trivial new vectors of $\Pi_{w}^{\prime}$ do not vanish. Observe that we may choose the same element for all $\sigma$-twists, i.e., such that $t_{\Pi_{w}^{\prime}}=t \sigma \Pi_{w}^{\prime}$. If $w \notin S\left(\Pi^{\prime}\right)$, then we may take $t_{\Pi_{w}^{\prime}}:=\mathrm{id}$. Depending on these choices, for all $w \in S(\mathcal{K})_{f}$, we let

$$
\xi_{\Pi_{w}^{\prime}}:=\text { the unique new vector such that } \xi_{\Pi_{w}^{\prime}}\left(t_{\Pi_{w}^{\prime}}\right)=1
$$

This pins down a special Whittaker vector $\xi_{\Pi_{f}^{\prime}}:=\otimes_{w \in S(\mathcal{K})_{f}}^{\prime} \xi_{\Pi_{w}^{\prime}} \in W\left(\Pi_{f}^{\prime}\right)$.

Our choice for $\Pi_{w}$ will depend on the data fixed for $\Pi_{w}^{\prime}$. First, we fix an element $t_{\Pi_{w}} \in T\left(\mathcal{K}_{w}\right)^{+}$analogously as for $G^{\prime}\left(\mathcal{K}_{w}\right)$. Now, for $w \notin S:=S\left(\Pi^{\prime}\right) \cup S(\psi)$, we let $\xi_{\Pi_{w}}$ be the unique new vector of $\Pi_{w}$, which satisfies $\xi_{\Pi_{w}}\left(t_{\Pi_{w}}\right)=1$. It is a certain non-zero multiple $c_{\Pi_{w}}$ of the essential vector, cf. [27, Théorème (4.1)]. If $w \in S_{f}$, we take $\xi_{\Pi_{w}}$ to be the unique Whittaker vector, whose restriction to $\iota\left(G^{\prime}\left(\mathcal{K}_{w}\right)\right)$ is supported on $N^{\prime}\left(\mathcal{K}_{w}\right) t_{\Pi_{w}^{\prime}} K^{\prime}\left(m_{w}^{\prime}\right)$ and there equal to $\psi_{w}^{-1} \omega_{\Pi_{w}^{\prime}}^{-1}$. This gives a special Whittaker vector $\xi_{\Pi_{f}}:=\otimes_{w \in S(\mathcal{K})_{f}}^{\prime} \xi_{\Pi_{w}} \in W\left(\Pi_{f}\right)$.

Lemma 3.7. Let $\xi_{\Pi_{f}}$ and $\xi_{\Pi_{f}^{\prime}}$ be the above Whittaker vectors. For a non-archimedean place $w$ of $\mathcal{K}$, the integral

$$
\Psi\left(s, \xi_{\Pi_{w}}, \xi_{\Pi_{w}^{\prime}}\right)=\int_{N^{\prime}\left(\mathcal{K}_{w}\right) \backslash G^{\prime}\left(\mathcal{K}_{w}\right)} \xi_{\Pi_{w}}(\iota(g)) \xi_{\Pi_{w}^{\prime}}(g)\|\operatorname{det}(g)\|_{w}^{s-\frac{1}{2}} d g_{w}
$$


converges for $\operatorname{Re}(s) \geqslant 1-\mathrm{m}$ and has a meromorphic continuation to all of $\mathbb{C}$. It equals

$$
\Psi\left(s, \xi_{\Pi_{w}}, \xi_{\Pi_{w}^{\prime}}\right)= \begin{cases}c_{\Pi_{w}} \cdot L\left(s, \Pi_{w} \times \Pi_{w}^{\prime}\right) & \text { if } w \notin S \\ \left\|\operatorname{det}\left(t_{\Pi_{w}^{\prime}}\right)\right\|_{w}^{s-\frac{1}{2}} \cdot \operatorname{vol}\left(K^{\prime}\left(m_{w}^{\prime}\right)\right) & \text { if } w \in S\end{cases}
$$

For all $\sigma \in \operatorname{Aut}(\mathbb{C}),{ }^{\sigma}\left(\xi_{\Pi_{w}^{\prime}}\right)=\xi_{\sigma} \Pi_{w}^{\prime}$ for all non-archimedean places $w$ of $\mathcal{K}$, whereas

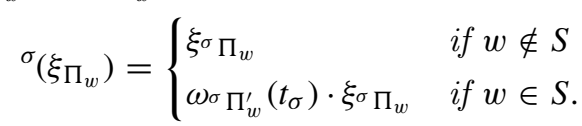

Proof. Any one of the above assertions is either well known or follows from a direct calculation using the definition of the local Whittaker vectors.

\subsection{An archimedean non-vanishing result}

Suppose from now on that $d g_{\infty}$ is the unique Haar measure on $G^{\prime}(\mathbb{C})$, which gives $U(n-1)$ volume 1 . Recall our choices of cohomology classes $\left[\Pi_{\infty}\right]$ and $\left[\Pi_{\infty}^{\prime}\right]$ from $\S 3.3$. Since $\xi_{\Pi_{\infty}, \underline{i}, \alpha}$ is $K$-finite and $\xi_{\Pi_{\infty}^{\prime}, \underline{j}, \beta}$ is $K^{\prime}$-finite, the integral

$$
\Psi\left(s, \xi_{\Pi_{\infty}, \underline{i}, \alpha}, \xi_{\Pi_{\infty}^{\prime}, \underline{j}, \beta}\right)=\int_{N^{\prime}(\mathbb{C}) \backslash G^{\prime}(\mathbb{C})} \xi_{\Pi_{\infty}, \underline{i}, \alpha}(\iota(g)) \xi_{\Pi_{\infty}^{\prime}, \underline{j}, \beta}(g)\|\operatorname{det}(g)\|_{\infty}^{s-\frac{1}{2}} d g_{\infty}
$$

converges for $\operatorname{Re}(s) \gg 0$. By [7, Theorem 1.2.(i)], $\Psi\left(s, \xi_{\Pi_{\infty}, \underline{i}, \alpha}, \xi_{\Pi_{\infty}^{\prime}, j, \beta}\right)$ is holomorphic at all critical values of $L\left(s, \Pi \times \Pi^{\prime}\right)$. Assume that $\frac{1}{2} \in \operatorname{Crit}\left(\Pi \times \Pi^{\prime}\right)$. In this case, we define

$$
c\left(\frac{1}{2}, \Pi_{\infty}, \Pi_{\infty}^{\prime}\right):=\sum_{\alpha, \beta} \sum_{\underline{i}, \underline{j}} s(\underline{i}, \underline{j}) \cdot \mathcal{T}\left(e_{\alpha} \otimes e_{\beta}^{\prime}\right) \cdot \Psi\left(\frac{1}{2}, \xi_{\Pi_{\infty}, \underline{i}, \alpha}, \xi_{\Pi_{\infty}^{\prime}, \underline{j}, \beta}\right),
$$

where $s(\underline{i}, \underline{j})$ is given by $\iota\left(X_{\underline{i}}^{*}\right) \wedge p\left(X_{\underline{j}}^{*}\right)=s(\underline{i}, \underline{j}) \cdot X_{1}^{*} \wedge \cdots \wedge X_{r}^{*}, r=\operatorname{dim}_{\mathbb{R}} \tilde{S}_{n-1}$. By what we said above, $c\left(\frac{1}{2}, \Pi_{\infty}, \Pi_{\infty}^{\prime}\right)$ is well defined, i.e., finite.

Now, drop the assumption that $\frac{1}{2} \in \operatorname{Crit}\left(\Pi \times \Pi^{\prime}\right)$, and let $s=\frac{1}{2}+m \in \operatorname{Crit}\left(\Pi \times \Pi^{\prime}\right)$ be an arbitrary critical point of $L\left(s, \Pi \times \Pi^{\prime}\right)$ with $m \geqslant 0$. Then, $\frac{1}{2} \in \operatorname{Crit}\left(\left(\Pi\|\cdot\|^{m}\right) \times \Pi^{\prime}\right)$, and we define

$$
c\left(\frac{1}{2}+m, \Pi_{\infty}, \Pi_{\infty}^{\prime}\right):=c\left(\frac{1}{2}, \Pi_{\infty}\|\cdot\|_{\infty}^{m}, \Pi_{\infty}^{\prime}\right)
$$

By our compatible choice of $\mathcal{T}^{(m)}$, this is well defined. The following theorem recently proved by Binyong Sun [44, Theorem A] is of the highest importance for the present paper.

Theorem 3.8. For all $s=\frac{1}{2}+m \in \operatorname{Crit}\left(\Pi \times \Pi^{\prime}\right)$ with $m \geqslant 0, c\left(\frac{1}{2}+m, \Pi_{\infty}, \Pi_{\infty}^{\prime}\right) \neq 0$.

Proof. Since Sun's theorem concerns properties of a non-trivial pairing $\operatorname{Hom}_{G^{\prime}(\mathbb{C})}\left(\Pi_{\infty} \otimes\right.$ $\left.\Pi_{\infty}^{\prime}, \mathbb{C}\right)$, and makes no reference to zeta integrals, Whittaker functions, or coefficient systems, we explain how to translate his theorem into our non-vanishing statement. We return to the notation of 3.3. Write $\mathfrak{p}=\mathfrak{g} / \mathfrak{k}$ and $\mathfrak{p}^{\prime}=\mathfrak{g}^{\prime} / \mathfrak{k}^{\prime}$. The expressions there for the generators $\left[\Pi_{\infty}\right] \in H^{b_{n}}\left(\mathfrak{g}, K, W\left(\Pi_{\infty}\right) \otimes E_{\mu}\right)$ and $\left[\Pi_{\infty}^{\prime}\right] \in H^{b_{n-1}}\left(\mathfrak{g}^{\prime}, K^{\prime}, W\left(\Pi_{\infty}^{\prime}\right) \otimes E_{\lambda}\right)$ are based on the following identifications:

$$
\begin{gathered}
H_{n}:=H^{b_{n}}\left(\mathfrak{g}, K, W\left(\Pi_{\infty}\right) \otimes E_{\mu}\right)=\operatorname{Hom}_{K}\left(\wedge^{b_{n}} \mathfrak{p} \otimes E_{\mu}^{*}, W\left(\Pi_{\infty}\right)\right), \\
H_{n-1}:=H^{b_{n-1}}\left(\mathfrak{g}^{\prime}, K^{\prime}, W\left(\Pi_{\infty}^{\prime}\right) \otimes E_{\lambda}\right)=\operatorname{Hom}_{K^{\prime}}\left(\wedge^{b_{n-1}} \mathfrak{p} \otimes E_{\lambda}^{*}, W\left(\Pi_{\infty}^{\prime}\right)\right) .
\end{gathered}
$$


Letting $h_{n}, h_{n-1}$ denote generators of the one-dimensional spaces $H_{n}$ and $H_{n-1}$, respectively, it is known that $\rho=\operatorname{Im}\left(h_{n}\right)$ (respectively, $\rho^{\prime}=\operatorname{Im}\left(h_{n-1}\right)$ ) is an irreducible $K$-type in $W\left(\Pi_{\infty}\right)$ (respectively, $K^{\prime}$-type in $\left.W\left(\Pi_{\infty}^{\prime}\right)\right)$. Sun's theorem states that, if $u$ is a generator of the one-dimensional space $\left.\operatorname{Hom}_{G^{\prime}(\mathbb{C})}\left(\overline{W\left(\Pi_{\infty}\right) \otimes W\left(\Pi_{\infty}^{\prime}\right.}\right), \mathbb{C}\right)$, where $\overline{W\left(\Pi_{\infty}\right) \otimes W\left(\Pi_{\infty}^{\prime}\right)}$ denotes the corresponding Casselman-Wallach (Frechet) completion, then $\left.u\right|_{\rho \otimes \rho^{\prime}} \neq 0$. This implies in particular that the Rankin-Selberg integral

$$
\Psi_{m}\left(\xi \otimes \xi^{\prime}\right):=\Psi\left(\frac{1}{2}+m, \xi, \xi^{\prime}\right)=\left[\int_{N^{\prime}(\mathbb{C}) \backslash G^{\prime}(\mathbb{C})} \xi(\iota(g)) \xi^{\prime}(g)\|\operatorname{det}(g)\|_{\infty}^{s-\frac{1}{2}} d g_{\infty}\right]_{s=\frac{1}{2}+m}
$$

defines a non-zero linear form on $\rho \otimes \rho^{\prime}$. Here, we are using the fact, due to Jacquet, Shalika, and Piatetski-Shapiro, that the Rankin-Selberg integral is not identically zero.

Now, if we let $\left\{e_{\alpha}^{*}\right\}$ and $\left\{e_{\beta}^{\prime, *}\right\}$ denote the dual bases to the bases $\left\{e_{\alpha}\right\}$ and $\left\{e_{\beta}^{\prime}\right\}$ of $E_{\mu}$ and $E_{\lambda}$ introduced in 3.3, we find that (in the obvious notation)

$$
\xi_{\Pi_{\infty}, \underline{i}, \alpha}=h_{n}\left(X_{\underline{i}} \otimes e_{\alpha}^{*}\right) \in \rho \quad \xi_{\Pi_{\infty}^{\prime}, \underline{j}, \beta}=h_{n-1}\left(X_{\underline{j}} \otimes e_{\beta}^{\prime, *}\right) \in \rho^{\prime}
$$

are the matrix coefficients of $h_{n}$ and $h_{n-1}$ in the chosen bases. Then the non-vanishing of $c\left(\frac{1}{2}+m, \Pi_{\infty}, \Pi_{\infty}^{\prime}\right)$ comes down to the non-vanishing of $\Psi_{m}$ on $\rho \otimes \rho^{\prime}$.

Observe that Theorem 3.8 implies that $c\left(\frac{1}{2}+m,{ }^{\sigma} \Pi_{\infty},{ }^{\sigma} \Pi_{\infty}^{\prime}\right) \neq 0$, for all $\sigma \in \operatorname{Aut}(\mathbb{C})$. We denote by $p\left(m,{ }^{\sigma} \Pi_{\infty},{ }^{\sigma} \Pi_{\infty}^{\prime}\right)$ the inverse of $c\left(\frac{1}{2}+m,{ }^{\sigma} \Pi_{\infty},{ }^{\sigma} \Pi_{\infty}^{\prime}\right)$. In the special case when $m=0$, we will abbreviate $p\left({ }^{\sigma} \Pi_{\infty},{ }^{\sigma} \Pi_{\infty}^{\prime}\right):=p\left(0,{ }^{\sigma} \Pi_{\infty},{ }^{\sigma} \Pi_{\infty}^{\prime}\right)$.

\subsection{A theorem on Whittaker periods}

Let us recall the Gaußsum of a Hecke character $\omega: \mathbb{Q}^{\times} \backslash \mathbb{A}_{\mathbb{Q}}^{\times} \rightarrow \mathbb{C}^{\times}$. Let $\mathfrak{c}$ stand for the conductor ideal of $\omega_{f}$. We take $y=\left(y_{p}\right)_{p \in S_{f}} \in \mathbb{A}_{f}^{\times} \operatorname{such}$ that $\operatorname{ord}_{p}\left(y_{p}\right)=-\operatorname{ord}_{p}(\mathfrak{c})$. Then, the Gauß sum of $\omega_{f}$ is defined as $\mathcal{G}\left(\omega_{f}, \psi_{f}, y\right)=\prod_{p \in S_{f}} \mathcal{G}\left(\omega_{p}, \psi_{p}, y_{p}\right)$, where the local Gauß sum $\mathcal{G}\left(\omega_{p}, \psi_{p}, y_{p}\right)$ is defined as

$$
\mathcal{G}\left(\omega_{p}, \psi_{p}, y_{p}\right)=\int_{\mathbb{Z}_{p}^{\times}} \omega_{p}\left(u_{p}\right)^{-1} \psi_{p}\left(y_{p} u_{p}\right) d u_{p}
$$

If all inputs are unramified at $p$, then $\mathcal{G}\left(\omega_{p}, \psi_{p}, y_{p}\right)=1$, so the above product is finite. Suppressing the dependence on $\psi$ and $y$, we denote $\mathcal{G}\left(\omega_{f}, \psi_{f}, y\right)$ simply by $\mathcal{G}\left(\omega_{f}\right)$.

If $\omega: \mathcal{K}^{\times} \backslash \mathbb{A}_{\mathcal{K}}^{\times} \rightarrow \mathbb{C}^{\times}$is a Hecke character of $\mathcal{K}$, let $\omega_{0}$ denote its restriction to the idèles of $\mathbb{Q}$. We are now ready to state and prove the main result of this section, which is analogous to the main result in [39].

Theorem 3.9. Let $\Pi=B C(\pi)\|\cdot\|^{\mathrm{m}}$ be a cuspidal automorphic representation of $G\left(\mathbb{A}_{\mathcal{K}}\right)$, $\mathrm{m} \in \mathbb{Z}$, and let $\Pi^{\prime}=B C\left(\pi^{\prime}\right)$ be an abelian automorphic representation of $G^{\prime}\left(\mathbb{A}_{\mathcal{K}}\right)$ obtained by base change from unitary groups as in $\S \S 2.4$ and 2.5. In particular, $\Pi$ is cohomological 
with respect to $E_{\mu}$, and $\Pi^{\prime}$ is cohomological with respect to $E_{\lambda}$. We assume that the highest weights $\mu$ and $\lambda$ satisfy Hypothesis 2.3. In view of the archimedean non-vanishing result, cf. Theorem 3.8, the following hold.

(1) For all critical values $\frac{1}{2}+m \in \operatorname{Crit}\left(\Pi \times \Pi^{\prime}\right)$ with $m \geqslant 0$ and every $\sigma \in \operatorname{Aut}(\mathbb{C})$,

$$
\sigma\left(\frac{L\left(\frac{1}{2}+m, \Pi_{f} \times \Pi_{f}^{\prime}\right)}{p(\Pi) p\left(\Pi^{\prime}\right) p\left(m, \Pi_{\infty}, \Pi_{\infty}^{\prime}\right) \mathcal{G}\left(\omega_{\Pi_{f, 0}^{\prime}}\right)}\right)=\frac{L\left(\frac{1}{2}+m,{ }^{\sigma} \Pi_{f} \times{ }^{\sigma} \Pi_{f}^{\prime}\right)}{p\left({ }^{\sigma} \Pi\right) p\left({ }^{\sigma} \Pi^{\prime}\right) p\left(m,{ }^{\sigma} \Pi_{\infty},{ }^{\sigma} \Pi_{\infty}^{\prime}\right) \mathcal{G}\left(\omega_{\sigma} \Pi_{f, 0}^{\prime}\right)} .
$$

$$
L\left(\frac{1}{2}+m, \Pi_{f} \times \Pi_{f}^{\prime}\right) \sim_{\mathbb{Q}\left(\Pi_{f}\right) \mathbb{Q}\left(\Pi_{f}^{\prime}\right)} p(\Pi) p\left(\Pi^{\prime}\right) p\left(m, \Pi_{\infty}, \Pi_{\infty}^{\prime}\right) \mathcal{G}\left(\omega_{\Pi_{f, 0}^{\prime}}\right),
$$

where $\sim_{\mathbb{Q}\left(\Pi_{f}\right) \mathbb{Q}\left(\Pi_{f}^{\prime}\right)}$ means up to multiplication by an element in the composition of number fields $\mathbb{Q}\left(\Pi_{f}\right) \mathbb{Q}\left(\Pi_{f}^{\prime}\right)$.

Remark 3.10. In fact, the central character of $\Pi^{\prime}$ is the twisted base change of the central character of $\pi^{\prime}$, and therefore its restriction to the idèles of $\mathbb{Q}$ is trivial! The term $\mathcal{G}\left(\omega_{\sigma} \Pi_{f, 0}^{\prime}\right)$ is nevertheless retained in the statement of the above theorem because it applies, with minor modifications, to cohomological automorphic representations not obtained by base change, in which case the presence of the Gauß sum, as in [39], is indispensable.

Proof. Since (1) implies (2) by Strong Multiplicity One for isobaric automorphic representations, we only prove (1). We will proceed in two steps.

Step 1: Assume $\frac{1}{2}$ is critical.

For any $\underline{i}, \underline{j}, \alpha$ and $\beta$, denote

$$
\varphi_{\Pi, i, \alpha}:=W^{-1}\left(\xi_{\Pi_{\infty}, \underline{i}, \alpha} \otimes \xi_{\Pi_{f}}\right) \in \Pi \quad \text { and } \quad \varphi_{\Pi^{\prime}, \underline{j}, \beta}:=W^{-1}\left(\xi_{\Pi_{\infty}^{\prime}, \underline{j}, \beta} \otimes \xi_{\Pi_{f}^{\prime}}\right) \in \Pi^{\prime},
$$

i.e., the inverse image of our particular Whittaker vectors. It follows directly from the definition of the various maps in our diagram that, if we chase $\xi_{\Pi_{f}} \times \xi_{\Pi_{f}^{\prime}}$ through the diagram, we obtain

$$
\begin{aligned}
\operatorname{Dia}\left(\xi_{\Pi_{f}} \times \xi_{\Pi_{f}^{\prime}}\right)= & \sum_{\alpha, \beta} \sum_{\underline{i}, \underline{j}} p(\Pi)^{-1} p\left(\Pi^{\prime}\right)^{-1} \cdot s(\underline{i}, \underline{j}) \cdot \mathcal{T}\left(e_{\alpha} \otimes e_{\beta}^{\prime}\right) \\
& \left.\cdot \int_{G^{\prime}(\mathcal{K}) \backslash G^{\prime}\left(\mathbb{A}_{\mathcal{K}}\right)} \varphi_{\Pi, \underline{i}, \alpha}\right|_{G^{\prime}\left(\mathbb{A}_{\mathcal{K}}\right)} \cdot \varphi_{\Pi^{\prime}, \underline{j}, \beta} d g,
\end{aligned}
$$

where $d g=d g_{\infty} \cdot d g_{f}$ (cf. $\S \S 3.8$ and 3.10). We are left to compute the latter integral. Since $\varphi_{\Pi, i, \alpha}$ is cuspidal, it is well known that

$$
\int_{G^{\prime}(\mathcal{K}) \backslash G^{\prime}(\mathbb{A} \mathcal{K})} \varphi_{\Pi, \underline{i}, \alpha}(\iota(g)) \cdot \varphi_{\Pi^{\prime}, \underline{j}, \beta}(g)\|\operatorname{det}(g)\|^{s-\frac{1}{2}} d g
$$

converges for all $s \in \mathbb{C}$ and equals

$$
\int_{N^{\prime}\left(\mathbb{A}_{\mathcal{K}}\right) \backslash G^{\prime}\left(\mathbb{A}_{\mathcal{K}}\right)}\left(\xi_{\Pi_{\infty}, \underline{i}, \alpha} \otimes \xi_{\Pi_{f}}\right)(\iota(g)) \cdot\left(\xi_{\Pi_{\infty}^{\prime}, j, \beta} \otimes \xi_{\Pi_{f}^{\prime}}\right)(g)\|\operatorname{det}(g)\|^{s-\frac{1}{2}} d g
$$


for $\operatorname{Re}(s) \gg 0$. For such $s$, this is furthermore equal to

$$
\Psi\left(s, \xi_{\Pi_{\infty}, \underline{i}, \alpha}, \xi_{\Pi_{\infty}^{\prime}, j, \beta}\right) \cdot L^{S}\left(s, \Pi \times \Pi^{\prime}\right) \prod_{w \notin S} c_{\Pi_{w}} \prod_{w \in S_{f}}\left\|\operatorname{det}\left(t_{\Pi_{w}^{\prime}}\right)\right\|_{w}^{s-\frac{1}{2}} \operatorname{vol}\left(K^{\prime}\left(m_{w}^{\prime}\right)\right)
$$

with $S=S\left(\Pi^{\prime}\right) \cup S(\psi)$ by Lemma 3.7. By analytic continuation, we obtain

$$
\operatorname{Dia}\left(\xi_{\Pi_{f}} \times \xi_{\Pi_{f}^{\prime}}\right)=\frac{L\left(\frac{1}{2}, \Pi_{f} \times \Pi_{f}^{\prime}\right)}{p(\Pi) p\left(\Pi^{\prime}\right) p\left(\Pi_{\infty}, \Pi_{\infty}^{\prime}\right)} \cdot \frac{\prod_{w \notin S} c_{\Pi_{w}} \prod_{w \in S_{f}} \operatorname{vol}\left(K^{\prime}\left(m_{w}^{\prime}\right)\right)}{\prod_{w \in S} L\left(\frac{1}{2}, \Pi_{w} \times \Pi_{w}^{\prime}\right)},
$$

so

$$
\sigma\left(\operatorname{Dia}\left(\xi_{\Pi_{f}} \times \xi_{\Pi_{f}^{\prime}}\right)\right)=\sigma\left(\frac{L\left(\frac{1}{2}, \Pi_{f} \times \Pi_{f}^{\prime}\right)}{p(\Pi) p\left(\Pi^{\prime}\right) p\left(\Pi_{\infty}, \Pi_{\infty}^{\prime}\right)}\right) \cdot \frac{\prod_{w \notin S} \sigma\left(c_{\Pi_{w}}\right) \prod_{w \in S_{f}} \operatorname{vol}\left(K^{\prime}\left(m_{w}^{\prime}\right)\right)}{\prod_{w \in S_{f}} \sigma\left(L\left(\frac{1}{2}, \Pi_{w} \times \Pi_{w}^{\prime}\right)\right)} .
$$

Here we note that, by our choice of a measure, cf. $\S 3.8, \prod_{w \in S_{f}} \operatorname{vol}\left(K^{\prime}\left(m_{w}^{\prime}\right)\right)$ is a rational number. Next, observe that $\sigma\left(L\left(\frac{1}{2}, \Pi_{w} \times \Pi_{w}^{\prime}\right)\right)=L\left(\frac{1}{2},{ }^{\sigma} \Pi_{w} \times{ }^{\sigma} \Pi_{w}^{\prime}\right)$, which is proved in Raghuram [39, Proposition 3.17]. Moreover, since the results of Jacquet, Piatetski-Shapiro, and Shalika in [27, 28] as well as Clozel [5, Lemme 4.6] are valid for $\Pi_{w}, w \notin S(\psi)$, the proof of Mahnkopf [35, Proposition 2.3.(c)] may be carried over to the situation considered here. In other words, $\sigma\left(c_{\Pi_{w}}\right)=c^{\sigma} \Pi_{w}$ for all $w \notin S$, cf. [34, p. 621] or [39, Proposition 3.21]. Finally, we obtain

$$
\sigma\left(\operatorname{Dia}\left(\xi_{\Pi_{f}} \times \xi_{\Pi_{f}^{\prime}}\right)\right)=\sigma\left(\frac{L\left(\frac{1}{2}, \Pi_{f} \times \Pi_{f}^{\prime}\right)}{p(\Pi) p\left(\Pi^{\prime}\right) p\left(\Pi_{\infty}, \Pi_{\infty}^{\prime}\right)}\right) \cdot \frac{\prod_{w \notin S} c^{\sigma} \Pi_{w} \prod_{w \in S_{f}} \operatorname{vol}\left(K^{\prime}\left(m_{w}^{\prime}\right)\right)}{\prod_{w \in S_{f}} L\left(\frac{1}{2},{ }^{\sigma} \Pi_{w} \times{ }^{\sigma} \Pi_{w}^{\prime}\right)}
$$

On the other hand, as all maps in the definition of our diagram are $\sigma$-equivariant, we see that $\sigma\left(\operatorname{Dia}\left(\xi_{\Pi_{f}} \times \xi_{\Pi_{f}^{\prime}}\right)\right)=\operatorname{Dia}\left({ }^{\sigma} \xi_{\Pi_{f}} \times{ }^{\sigma} \xi_{\Pi_{f}^{\prime}}\right)$. Therefore, Lemma 3.7 implies that

$$
\sigma\left(\operatorname{Dia}\left(\xi_{\Pi_{f}} \times \xi_{\Pi_{f}^{\prime}}\right)\right)=\left(\prod_{w \in S_{f}} \omega \sigma \Pi_{w}^{\prime}\left(t_{\sigma}\right)\right) \cdot \operatorname{Dia}\left(\xi^{\sigma} \Pi_{f} \times \xi_{\sigma} \Pi_{f}^{\prime}\right)
$$

As $\omega^{\sigma} \Pi_{w}^{\prime}$ is unramified outside $S\left({ }^{\sigma} \Pi^{\prime}\right)$, and since $t_{\sigma} \in \widehat{\mathcal{O}}^{*}$, we get $\omega^{\sigma} \Pi_{w}^{\prime}\left(t_{\sigma}\right)=1$ for $w \notin S\left({ }^{\sigma} \Pi^{\prime}\right)$. Hence, observing that $S\left(\Pi^{\prime}\right)=S\left({ }^{\sigma} \Pi^{\prime}\right)$, the finite product equals $\prod_{w \in S_{f}} \omega_{\sigma} \Pi_{w}^{\prime}\left(t_{\sigma}\right)=\omega_{\sigma} \Pi_{f}^{\prime}\left(t_{\sigma}\right)$. We claim that there is the identity

$$
\omega_{\sigma}{\Pi_{f}^{\prime}}\left(t_{\sigma}\right)=\frac{\sigma\left(\mathcal{G}\left(\omega_{\Pi_{f, 0}^{\prime}}\right)\right)}{\mathcal{G}\left(\omega_{\sigma} \Pi_{f, 0}^{\prime}\right)} .
$$

Indeed, at a place $p \in S_{f}$,

$$
\begin{aligned}
\mathcal{G}\left(\omega_{\sigma \Pi_{p, 0}^{\prime}}\right) & =\int_{\mathbb{Z}_{p}^{\times}} \omega_{\sigma \Pi_{p, 0}^{\prime}}\left(u_{p}\right)^{-1} \psi_{p}\left(y_{p} u_{p}\right) d u_{p} \\
& =\int_{\mathbb{Z}_{p}^{\times}} \sigma\left(\omega_{\Pi_{p, 0}^{\prime}}\left(u_{p}\right)\right)^{-1} \sigma\left(\psi_{p}\left(y_{p} u_{p} t_{\sigma}^{-1}\right)\right) d u_{p}
\end{aligned}
$$




$$
\begin{aligned}
& =\sigma\left(\omega_{\Pi_{p, 0}^{\prime}}\left(t_{\sigma}\right)\right)^{-1} \int_{\mathbb{Z}_{p}^{\times}} \sigma\left(\omega_{\Pi_{p, 0}^{\prime}}\left(u_{p}\right)^{-1} \psi_{p}\left(y_{p} u_{p}\right)\right) d u_{p} \\
& =\omega_{\sigma} \Pi_{p, 0}^{\prime}\left(t_{\sigma}\right)^{-1} \cdot \sigma\left(\int_{\mathbb{Z}_{p}^{\times}} \omega_{\Pi_{p, 0}^{\prime}}\left(u_{p}\right)^{-1} \psi_{p}\left(y_{p} u_{p}\right) d u_{p}\right) \\
& =\omega_{\sigma} \Pi_{p, 0}^{\prime}\left(t_{\sigma}\right)^{-1} \cdot \sigma\left(\mathcal{G}\left(\omega_{\Pi_{p, 0}^{\prime}}\right)\right),
\end{aligned}
$$

where the second last equation follows from the fact that - by the very choice of $\psi$ - the above integral is the finite sum over the classes modulo the conductor ideal of $\omega_{\Pi_{p}^{\prime}}$ (on which the integrand is constant). Therefore,

$$
\begin{aligned}
& \sigma\left(\frac{L\left(\frac{1}{2}, \Pi_{f} \times \Pi_{f}^{\prime}\right)}{p(\Pi) p\left(\Pi^{\prime}\right) p\left(\Pi_{\infty}, \Pi_{\infty}^{\prime}\right)}\right) \cdot \frac{\prod_{w \notin S} c^{\sigma} \Pi_{w} \prod_{w \in S_{f}} \operatorname{vol}\left(K^{\prime}\left(m_{w}^{\prime}\right)\right)}{\prod_{w \in S_{f}} L\left(\frac{1}{2},{ }^{\sigma} \Pi_{w} \times{ }^{\sigma} \Pi_{w}^{\prime}\right)} \\
& \quad=\frac{\sigma\left(\mathcal{G}\left(\omega_{\Pi_{f, 0}^{\prime}}\right)\right)}{\mathcal{G}\left(\omega_{\sigma} \Pi_{f, 0}^{\prime}\right)} \cdot \operatorname{Dia}\left(\xi^{\sigma} \Pi_{f} \times \xi^{\sigma} \Pi_{f}^{\prime}\right) .
\end{aligned}
$$

Recalling that $S\left(\Pi^{\prime}\right)=S\left({ }^{\sigma} \Pi^{\prime}\right)$ and that $m_{w}^{\prime}$ is the same for $\Pi_{w}^{\prime}$ and ${ }^{\sigma} \Pi_{w}^{\prime}$, we obtain, by what we have seen above, that

$$
\operatorname{Dia}\left(\xi^{\sigma} \Pi_{f} \times \xi_{\sigma} \Pi_{f}^{\prime}\right)=\frac{L\left(\frac{1}{2},{ }^{\sigma} \Pi_{f} \times{ }^{\sigma} \Pi_{f}^{\prime}\right)}{p\left(^{\sigma} \Pi\right) p\left({ }^{\sigma} \Pi^{\prime}\right) p\left({ }^{\sigma} \Pi_{\infty},{ }^{\sigma} \Pi_{\infty}^{\prime}\right)} \cdot \frac{\prod_{w \notin S} c{ }^{\sigma} \Pi_{w} \prod_{w \in S_{f}} \operatorname{vol}\left(K^{\prime}\left(m_{w}^{\prime}\right)\right)}{\prod_{w \in S_{f}} L\left(\frac{1}{2},{ }^{\sigma} \Pi_{w} \times{ }^{\sigma} \Pi_{w}^{\prime}\right)}
$$

Comparing the last two equations shows (1) if $\frac{1}{2}$ is critical.

Step 2: The general case.

We drop the assumption that $\frac{1}{2}$ is critical now. Let $s=\frac{1}{2}+m$ be an arbitrary critical value of $L\left(s, \Pi \times \Pi^{\prime}\right), m \geqslant 0$. Then $\frac{1}{2}$ is critical for $L\left(s,\left(\Pi\|\cdot\|^{m}\right) \times \Pi^{\prime}\right)$, and we are in the situation considered above. Hence, by what we have just observed,

$$
\begin{gathered}
\sigma\left(\frac{L\left(\frac{1}{2},\left(\Pi\|\cdot\|^{m}\right)_{f} \times \Pi_{f}^{\prime}\right)}{p\left(\Pi\|\cdot\|^{m}\right) p\left(\Pi^{\prime}\right) p\left(\Pi_{\infty}\|\cdot\|_{\infty}^{m}, \Pi_{\infty}^{\prime}\right) \mathcal{G}\left(\omega_{\left.\Pi_{f, 0}^{\prime}\right)}\right)}\right) \\
=\frac{L\left(\frac{1}{2},{ }^{\sigma}\left(\Pi\|\cdot\|^{m}\right)_{f} \times{ }^{\sigma} \Pi_{f}^{\prime}\right)}{p\left({ }^{\sigma}\left(\Pi\|\cdot\|^{m}\right)\right) p\left({ }^{\sigma} \Pi^{\prime}\right) p\left({ }^{\sigma}\left(\Pi_{\infty}\|\cdot\|_{\infty}^{m}\right),{ }^{\sigma} \Pi_{\infty}^{\prime}\right) \mathcal{G}\left(\omega_{\sigma} \Pi_{f, 0}^{\prime}\right)} \\
=\frac{L\left(\frac{1}{2}+m,{ }^{\sigma} \Pi_{f} \times{ }^{\sigma} \Pi_{f}^{\prime}\right)}{p\left({ }^{\sigma} \Pi\|\cdot\|^{m}\right) p\left({ }^{\sigma} \Pi^{\prime}\right) p\left(m,{ }^{\sigma} \Pi_{\infty},{ }^{\sigma} \Pi_{\infty}^{\prime}\right) \mathcal{G}\left(\omega_{\sigma} \Pi_{f, 0}^{\prime}\right)}
\end{gathered}
$$

By [40, Theorem 4.1], the period for $\Pi_{f}$ satisfies the relation

$$
\sigma\left(\frac{p\left(\Pi\|\cdot\|^{m}\right)}{p(\Pi)}\right)=\frac{p\left({ }^{\sigma} \Pi\|\cdot\|^{m}\right)}{p\left({ }^{\sigma} \Pi\right)},
$$

where we used that $\mathcal{G}\left(\|\cdot\|_{f}^{m}\right)=1$. This proves the theorem. 
Remark 3.11. At no time in the proof did we use the assumption that $\Pi^{\prime}$ is of abelian type, other than to avoid introducing additional notation. It is therefore clear that our result also holds for cuspidal automorphic representations $\Pi^{\prime}$ of $G^{\prime}\left(\mathbb{A}_{\mathcal{K}}\right)$; in other words, the proof of Theorem 3.9 also yields Theorem 1.3. As mentioned in the introduction, in this case, i.e., if $\Pi^{\prime}$ is cuspidal, it has recently also been proved by Raghuram in [38, Theorem 1.1] - even over any number field. Moreover, it is clear that we did not need that $\Pi$ and $\Pi^{\prime}$ are constructed via base change in order to prove Theorem 3.9. However, for later use, and to simplify the determination of the set of critical points, we already assumed this to be the case.

Remark 3.12. Let us finally also remark that we did not need to fix some open compact subgroup $K_{f} \subset G\left(\mathbb{A}_{f}\right)$ within the entire process of showing Theorem 3.9.

\section{Motives - an interlude}

This section reviews and extends the results of $[20,23]$ that are needed to prove the main results of this paper. The crucial definitions and calculations are scattered in different parts of the two papers in question, allowing space for several confusing sign changes, and their conventions are moreover not quite compatible. We hope that the present section will provide a more convenient reference. Moreover, the results of the earlier papers were proved under unnecessarily restrictive hypotheses; here we have striven for maximum generality, always assuming that the base field is an imaginary quadratic field.

The main purpose of this section is to compare the results on special values proved by automorphic methods to Deligne's conjecture, stated in the setting of motives for absolute Hodge cycles. This setting is partially hypothetical. It is not known, for example, that two geometric realizations in the cohomology of Shimura varieties of the Galois representations attached to a (motivic) algebraic Hecke character are isomorphic as motives, and in particular that they define the same periods. We are therefore led to introduce automorphic analogues of the invariants that arise in the (motivic) calculation of Deligne's periods over imaginary quadratic fields. See also [48] for calculations of Deligne periods of motives over $\mathbb{Q}$.

From now on we let $\mathrm{m}=0$, i.e., the cuspidal automorphic representation $\Pi$ is simply the unitary base change from $\pi$.

\subsection{Tensor products of motives}

The $L$-functions of the automorphic representations $\Pi$ and $\Pi^{\prime}$ of the previous section are conjecturally attached to motives over $M(\Pi)$ and $M\left(\Pi^{\prime}\right)$ over $\mathcal{K}$ of ranks $n$ and $n-1$, respectively, with coefficients in finite, possibly non-trivial extensions

$$
E(\Pi) / \mathbb{Q}\left(\Pi_{f}\right) \quad \text { and } \quad E\left(\Pi^{\prime}\right) / \mathbb{Q}\left(\Pi_{f}^{\prime}\right)
$$

The passage from $\Pi$ to $M(\Pi)$ involves a standard shift, thus

$$
L(s, M(\Pi))=L\left(s+\frac{1-n}{2}, \Pi\right)
$$


whose center of symmetry is at $s=\frac{n}{2}$ rather than at $s=\frac{1}{2}$. One can in any case attach Hodge structures and compatible families of $\ell$-adic representations to $\Pi$ and $\Pi^{\prime}$, pure of weight $n-1$ and $n-2$, respectively, so the tensor product $M(\Pi) \otimes_{\mathcal{K}} M\left(\Pi^{\prime}\right)$ is pure of weight $2 n-3$, and the center of symmetry of the functional equation of its $L$-function is at the point $s=n-1$.

More generally, we can take $\Pi^{\prime}$ to be an automorphic representation of $\mathrm{GL}_{n^{\prime}}\left(\mathbb{A}_{\mathcal{K}}\right)$. We will be particularly interested in the cases $n^{\prime}=n-1$ and $n^{\prime}=1$, but the methods of the present paper allow us to say quite a lot about the general case. In particular, the center of symmetry of the functional equation is the point $\frac{n+n^{\prime}-1}{2}$, which is not necessarily an integer; however, we will also be interested in critical values to the right of the center of symmetry. The fact that $\Pi$ and $\Pi^{\prime}$ arise by base change from unitary groups is reflected in the polarization property

$$
M(\Pi)^{c} \stackrel{\sim}{\rightarrow} M(\Pi)^{\vee}(1-n) \text { and } M\left(\Pi^{\prime}\right)^{c} \stackrel{\sim}{\rightarrow} M\left(\Pi^{\prime}\right)^{\vee}\left(1-n^{\prime}\right) .
$$

A formalism relating the various realizations of motives satisfying (4.1) and defining their period invariants is developed in $[23, \S 1.1]$, and the Deligne periods of the associated adjoint and tensor product motives are computed in [23, §§ 1.3-1.4].

Recall that the formula of Theorem 3.9 is valid provided the initial representations $\pi$ and $\pi^{\prime}$ of the definite unitary groups $H$ and $H^{\prime}$ are cohomological with respect to finite-dimensional representations satisfying the equivalent conditions of Lemma 2.1. Exactly the same conditions appear in $[23,(2.3 .1)]$ where they are used to evaluate the Deligne period of $M(\Pi) \otimes \mathcal{K} M\left(\Pi^{\prime}\right)$ - here $n^{\prime}=n-1$ - when the Ichino-Ikeda conjecture computes the central value $L\left(\frac{1}{2}, \Pi_{f} \times \Pi_{f}^{\prime}\right)$ in terms of periods of integrals of automorphic forms on $H \times H^{\prime}$. This is the same central value that appears on the left-hand side of the formulas in 3.9. We exploit this identification in subsequent sections. Here we complete [23] by determining the set of all critical values and their corresponding Deligne periods, when the inequalities of Lemma 2.1 are satisfied.

Write $M=M(\Pi), M^{\prime}=M\left(\Pi^{\prime}\right)$. As in [23], the Hodge types for $M$ (respectively $M^{\prime}$ ) are denoted

$$
\left(p_{i}, q_{i}\right):=\left(p_{i}, n-1-p_{i}\right) \quad \text { and } \quad\left(p_{i}^{c}, q_{i}^{c}\right):=\left(p_{i}^{c}, n-1-p_{i}^{c}\right)=\left(q_{n+1-i}, p_{n+1-i}\right)
$$

(respectively $\left(r_{j}, n^{\prime}-1-r_{j}\right)$ and $\left.\left(r_{j}^{c}, n^{\prime}-1-r_{j}^{c}\right)\right)$. Bearing in mind that the cohomological parameters of $\Pi$ and $\Pi^{\prime}$ are respectively given by the tuples $\mu$ and $\lambda$ as in 2.2 (and not by $a_{i}$ 's and $b_{j}$ 's as in [23]), we have

$$
p_{i}=n-i+\mu_{i}, \quad r_{j}=n^{\prime}-j+\lambda_{j} .
$$

For any integer $m$, the Hodge types for the Tate twist $M(m)$ are

$$
\begin{gathered}
\left(p_{i}-m, n-1-p_{i}-m\right) \text { and } \\
\left(p_{i}^{c}-m, n-1-p_{i}^{c}-m\right)=\left(n-1-p_{n+1-i}-m, p_{n+1-i}-m\right),
\end{gathered}
$$

where the equality on the right is a consequence of (4.1). We extend the definition of [23] by letting $T\left(M(m), M^{\prime}\right)$ be the set of pairs $(t, u)$ of indices, $1 \leqslant t \leqslant n, 1 \leqslant u \leqslant n^{\prime}$, such that $p_{t}^{c}-m+r_{u}^{c} \geqslant \frac{w+1}{2}$, and write $T\left(M, M^{\prime}\right)=T\left(M(0), M^{\prime}\right)$. Using (4.4), we transform 
the left hand side of this equation to

$$
\begin{aligned}
& n-1-p_{n+1-t}+n^{\prime}-1-r_{n^{\prime}+1-u}-m \geqslant \frac{n+n^{\prime}-1-2 m}{2} \\
& \quad \Leftrightarrow \frac{n+n^{\prime}-3}{2} \geqslant p_{n+1-t}+r_{n^{\prime}+1-u}
\end{aligned}
$$

and this is true if and only if

$$
\frac{n+n^{\prime}+1}{2} \geqslant t+u+\mu_{n+1-t}+\lambda_{n^{\prime}+1-u}
$$

\subsection{Critical values when $n^{\prime}=n-1$}

For the moment we let $n^{\prime}=n-1$. The tensor product motive we consider is $R\left(M \otimes M^{\prime}\right)=$ $R_{\mathcal{K} / \mathbb{Q}}\left(M \otimes M^{\prime}\right)$, of weight $w=2 n-3$. Now in view of 2.1 , we make the following

Remark 4.6. When $n^{\prime}=n-1, T\left(M, M^{\prime}\right)$ consists exactly of the set of pairs $\{(t, u) \mid t+$ $u \leqslant n\}$, of cardinality $\frac{n(n-1)}{2}$.

To calculate the Gamma factor, we follow Serre's recipe (see [8, §§ 5.2-5.3]) and find

$$
\prod_{a, b} \Gamma_{\mathbb{C}}\left(s-p_{a}-r_{b}\right) \times \prod_{t, u} \Gamma_{\mathbb{C}}\left(s-p_{t}^{c}-r_{u}^{c}\right)
$$

where the product is taken over pairs such that $p_{a}+r_{b} \leqslant \frac{w-1}{2}=n-2$ and $p_{t}^{c}+r_{u}^{c} \leqslant$ $n-2$. For the first set we have

$$
2 n-1-a-b+\mu_{a}+\lambda_{b} \leqslant n-2 \Leftrightarrow n+1+\mu_{a}+\lambda_{b} \leqslant a+b .
$$

By the branching rules this holds if and only if $a+b \geqslant n+1$. For the second set the polarization gives us

$$
\begin{aligned}
p_{t}^{c}+r_{u}^{c} & =2 n-3-\left(p_{n+1-t}+r_{n-u}\right) \leqslant n-2 \\
& \Leftrightarrow n-1 \leqslant p_{n+1-t}+r_{n-u} \\
& \Leftrightarrow n-1 \leqslant t+u-2+\mu_{n+1-t}+\lambda_{n-u}
\end{aligned}
$$

which is true if and only if $n+1 \leqslant t+u$.

There is therefore a pole at the integer $m$ unless $m \geqslant 1+\sup \left(\sup _{a+b \geqslant n+1} p_{a}+\right.$ $r_{b}, \sup _{t+u \geqslant n+1} p_{t}^{c}+r_{u}^{c}$ ). The inequalities 2.1 imply that it suffices to consider the pairs $(a, b)$ and $(t, u)$ with $a+b=n+1, t+u=n+1$. The minimum is thus

$$
m_{\min }=1+\sup \left(\sup _{a+b=n+1} n-2+\mu_{a}+\lambda_{b}, \sup _{t+u=n+1} n-2-\mu_{n+1-t}-\lambda_{n-u}\right) .
$$

This gives the lower bound of the critical set, and the functional equation that exchanges $s$ with $w+1-s$ implies the upper bound is 


$$
\begin{aligned}
m_{\max } & =w+1-m_{\min }=2 n-2-m_{\min } \\
& =\inf \left(n-1-\sup _{1 \leqslant a \leqslant n}\left(\mu_{a}+\lambda_{n+1-a}\right), n-1+\inf _{1 \leqslant t \leqslant n}\left(\mu_{u}+\lambda_{n-u}\right)\right) \\
& =n-1+\inf \left(-\sup _{2 \leqslant a \leqslant n}\left(\mu_{a}+\lambda_{n+1-a}\right), \inf _{1 \leqslant u \leqslant n-1}\left(\mu_{u}+\lambda_{n-u}\right)\right) .
\end{aligned}
$$

In the following lemma, $\mu(m)$ denotes the $n$-tuple $\mu_{1}-m \geqslant \mu_{2}-m \geqslant \cdots \geqslant \mu_{n}-m$, the highest weight of the representation $E_{\mu}^{\mathrm{unt}} \otimes \operatorname{det}^{-m}$.

Lemma 4.7. The set of critical points of the $L$-function $L\left(s, R\left(M \otimes M^{\prime}\right)\right)$ is the set of integers $s_{0}=n-1 \pm m$, where $m$ runs through the non-negative integers such that $\operatorname{Hom}_{G^{\prime}(\mathbb{C})}\left(\left(E_{\mu(m)}^{\mathrm{unt}} \otimes E_{\mu^{\mathrm{v}}(m)}^{\mathrm{unt}}\right) \otimes\left(E_{\lambda}^{\mathrm{unt}} \otimes E_{\lambda^{\mathrm{v}}}^{\mathrm{unt}}\right), \mathbb{C}\right) \neq 0$. The maximum value of $m$ is also the minimum of the distances between successive entries in the sequence of inequalities

$$
\mu_{1} \geqslant-\lambda_{n-1} \geqslant \mu_{2} \geqslant-\lambda_{n-2} \geqslant \cdots \geqslant-\lambda_{1} \geqslant \mu_{n} .
$$

Proof. The branching law implies that the existence of $G^{\prime}(\mathbb{C})$-equivariant homomorphisms

$$
E_{\mu(m)}^{\mathrm{unt}} \otimes E_{\lambda}^{\mathrm{unt}} \otimes \rightarrow \mathbb{C}, \quad E_{\mu^{\mathrm{v}}(m)}^{\mathrm{unt}} \otimes E_{\lambda^{\mathrm{v}}}^{\mathrm{unt}} \rightarrow \mathbb{C}
$$

is equivalent to the two series of inequalities

$$
\begin{aligned}
& \mu_{1}-m \geqslant-\lambda_{n-1} \geqslant \mu_{2}-m \geqslant-\lambda_{n-2} \geqslant \cdots \geqslant-\lambda_{1} \geqslant \mu_{n}-m, \\
& -\mu_{1}-m \leqslant \lambda_{n-1} \leqslant-\mu_{2}-m \leqslant \lambda_{n-2} \leqslant \cdots \leqslant \lambda_{1} \leqslant-\mu_{n}-m,
\end{aligned}
$$

which is equivalent to the determination of $m_{\max }$ above. The final sentence is clear.

We now derive an expression for $c^{+}\left(R\left(M \otimes M^{\prime}\right)(m)\right)$, following [23, $\left.\S 1.4\right]$, when $n^{\prime}=$ $n-1$. Recall the formula in [23, Lemma 1.4.1]:

$$
\begin{aligned}
c^{+}\left(R\left(M \otimes M^{\prime}\right)^{\vee}\right) & =c^{-}\left(R\left(M \otimes M^{\prime}\right)^{\vee}\right) \\
& \sim \prod_{(t, u) \in T\left(M, M^{\prime}\right)} Q_{n+1-t}(M)^{-1} Q_{n-u}\left(M^{\prime}\right)^{-1} \cdot \delta\left(M \otimes M^{\prime}\right)^{-1} \\
& \sim \prod_{t+u \leqslant n} Q_{n+1-t}(M)^{-1} Q_{n-u}\left(M^{\prime}\right)^{-1} \cdot \delta(M)^{1-n} \cdot \delta\left(M^{\prime}\right)^{-n} .
\end{aligned}
$$

Here $\delta(M)$ and $\delta\left(M^{\prime}\right)$ are the full period determinants studied in [23, $\left.\S 1.2\right]$, specifically in Lemma 1.2.7, which we recall here. We let $E(M)$ and $E\left(M^{\prime}\right)$ be the respective coefficient fields of $M$ and $M^{\prime}$.

Lemma 4.9. There are elements $d(M) \in E(M)$ and $d\left(M^{\prime}\right) \in E\left(M^{\prime}\right)$ such that

$$
\delta(M)^{-1} \sim d(M)^{\frac{1}{2}}(2 \pi i)^{n(n-1) / 2} Q_{\operatorname{det} M}^{1 / 2} \quad \text { and } \quad \delta\left(M^{\prime}\right)^{-1} \sim d\left(M^{\prime}\right)^{\frac{1}{2}}(2 \pi i)^{(n-1)(n-2) / 2} Q_{\operatorname{det} M^{\prime}}^{1 / 2},
$$

where $Q_{\operatorname{det} M}=\prod_{t=1}^{n} Q_{t}(M)$ and $Q_{\operatorname{det} M^{\prime}}=\prod_{u=1}^{n-1} Q_{u}\left(M^{\prime}\right)$.

We introduce the new invariants

$$
q(M):=d(M)^{\frac{1}{2}} Q_{\operatorname{det} M}^{1 / 2} \quad \text { and } \quad Q_{\leqslant r}(M):=\prod_{t \leqslant r} Q_{t}\left(M^{c}\right), \quad 1 \leqslant r \leqslant n
$$


and define $q\left(M^{\prime}\right)$ and $Q_{\leqslant r}\left(M^{\prime}\right)$ analogously. Recall [23, Remark 1.3.6]:

$$
Q_{t}\left(M^{c}\right) \sim Q_{n+1-t}(M)^{-1}, \quad t=1, \ldots, n .
$$

Combining this with (4.8)-(4.10), we obtain

$$
\begin{aligned}
& c^{ \pm}\left(R\left(M \otimes M^{\prime}\right)^{\vee}\right) \\
& \quad \sim(2 \pi i)^{\frac{n(n-1)^{2}}{2}}(2 \pi i)^{\frac{n(n-1)(n-2)}{2}} \prod_{t=1}^{n-1} Q_{t}\left(M^{c}\right)^{n-t} \prod_{u=1}^{n-1} Q_{u}\left(M^{\prime c}\right)^{n-u} \cdot q(M)^{n-1} q\left(M^{\prime}\right)^{n} \\
& \sim(2 \pi i)^{n(n-1)(2 n-3) / 2} \prod_{r=1}^{n-1}\left(Q_{\leqslant r}(M) \cdot Q_{\leqslant r}\left(M^{\prime}\right)\right) \cdot q(M)^{n-1} q\left(M^{\prime}\right)^{n} \\
& \sim(2 \pi i)^{n(n-1)(2 n-3) / 2} \prod_{r=1}^{n-1}\left[Q_{\leqslant r}(M) \cdot q(M)\right] \prod_{r^{\prime}=1}^{n-2}\left[Q_{\leqslant r^{\prime}}\left(M^{\prime}\right) \cdot q\left(M^{\prime}\right)\right]
\end{aligned}
$$

where we have used the relation $Q_{\leqslant n-1}\left(M^{\prime}\right) \sim q\left(M^{\prime}\right)^{-2}$ (cf. [23, Lemma 1.2.7] and [23, $(1.2 .5)])$ to obtain the more symmetric expression in the last line. Finally, the polarization gives us an isomorphism for each integer $m$ :

$$
R\left(M \otimes M^{\prime}\right)(m) \cong R\left(M \otimes M^{\prime}\right)^{\vee}(m+3-2 n) .
$$

We write $P_{\leqslant r}(M):=Q_{\leqslant r}(M) \cdot q(M)$, and likewise for $M^{\prime}$. It thus follows from [8], Formula (5.1.8) that

$$
\begin{aligned}
c^{+}\left(R\left(M \otimes M^{\prime}\right)(m)\right) & \sim(2 \pi i)^{n(n-1)(m+3-2 n)} c^{ \pm}\left(R\left(M \otimes M^{\prime}\right)^{\vee}\right) \\
& \sim(2 \pi i)^{\frac{n(n-1)}{2}(2 m-2 n+3)} \prod_{r=1}^{n-1} P_{\leqslant r}(M) \prod_{r^{\prime}=1}^{n-2} P_{\leqslant r^{\prime}}\left(M^{\prime}\right) .
\end{aligned}
$$

\subsection{Critical values when $n^{\prime}=1$}

We repeat the above calculation but now assume $n^{\prime}=1$ and weaken the polarization hypothesis for $M^{\prime}=M\left(\Pi^{\prime}\right)$. We assume that $M^{\prime}$ is of weight $-\kappa$ and that there is a Dirichlet character $\alpha_{0}$ and a non-degenerate pairing as in [20, (1.6.2)]:

$$
R\left(M^{\prime}\right) \otimes R\left(M^{\prime}\right) \rightarrow \mathbb{Q}\left(\alpha_{0} \cdot \varepsilon_{\mathcal{K}}\right)(\kappa)
$$

We assume moreover that there are Hecke characters $\chi$ and $\alpha$, as in [20] (especially $\S \S 2.9$ and 3.5), so that $\Pi^{\prime}=B C(\chi) \cdot \alpha$, with $\chi_{\infty}(z)=z^{-k}, \alpha_{\infty}(z)=z^{\kappa}$, so that $\Pi_{\infty}^{\prime}(z)=$ $(z / \bar{z})^{-k} \cdot z^{\kappa}$. The motive $R\left(M^{\prime}\right)$ then has Hodge types $(k-\kappa,-k) ;(-k, k-\kappa)$. (The parameter $\kappa$ is needed for parity considerations and will in practice either be 0 or 1 .) The restriction of $\Pi^{\prime}$ to the idèles of $\mathbb{Q}$ equals $\alpha_{0}$ multiplied by a power of the norm; thus the notation of 4.13 is consistent with that introduced on [20, p. 92].

The indices $b$ and $u$ only take the value 1 ; we have $r_{1}=k-\kappa$ and $r_{1}^{c}=-k$, and the weight of $R\left(M \otimes M^{\prime}\right)$ is $n-1-\kappa$. By definition $T\left(M(m), M^{\prime}\right)$ is the set of pairs $(t, u)=$ $(t, 1)$ such that $p_{t}^{c}-m-k \geqslant \frac{n-\kappa-2 m}{2}$. 
Remark 4.14. When $n^{\prime}=1, T\left(M(m), M^{\prime}\right)$ consists exactly of the set of pairs $(t, 1)$ such that

$$
n-2 p_{t}^{c} \leqslant-2 k+\kappa .
$$

Since the $p_{t}^{c}$ are decreasing, $T\left(M(m), M^{\prime}\right)$ is therefore the set of $t \leqslant s\left(M^{\prime}\right)$ where

$$
s\left(M^{\prime}\right):=s\left(M^{\prime}, M\right):=\sup \left\{t \mid n-2 p_{t}^{c} \leqslant-2 k+\kappa\right\}
$$

and it is of cardinality $s\left(M^{\prime}\right)$. Let

$$
r\left(M^{\prime}\right):=r\left(M^{\prime}, M\right):=n-s\left(M^{\prime}, M\right) .
$$

If we assume $\kappa \equiv n(\bmod 2)$, then, given the relations $p_{i}^{c}=n-1-p_{n+1-i}=q_{n+1-i}$, we find

$$
2 k-\kappa \in\left[n-2 p_{r\left(M^{\prime}\right)}, n-2 p_{r\left(M^{\prime}\right)+1}-2\right]=\left[n-2 q_{s\left(M^{\prime}\right)+1}^{c}, 2 p_{s\left(M^{\prime}\right)}^{c}-n\right] .
$$

The set of critical values is determined in [20], (3.3.8.1). When $n>1$ we choose $\kappa$ such that the weight $w$ of $R(M) \otimes R\left(M^{\prime}\right)$ is odd; then $\frac{w+1}{2}$ is always critical. When $n=n^{\prime}=1$ we take $\kappa=0$ (respectively $\kappa=1$ ) and $k \neq 0$; then the near-central point $s=1$ (respectively the central point $s=0$ ) is always critical. As in the previous section, we obtain the formula

$$
\begin{aligned}
c^{ \pm}\left(R\left(M \otimes M^{\prime}\right)^{\vee}\right) & \sim \prod_{t \leqslant s\left(M^{\prime}\right)} Q_{n+1-t}(M)^{-1} Q_{1}\left(M^{\prime}\right)^{-1} \cdot \delta\left(M \otimes M^{\prime}\right)^{-1} \\
& \sim \prod_{t \leqslant s\left(M^{\prime}\right)} Q_{t}\left(M^{c}\right) Q_{1}\left(M^{\prime}\right)^{-1} \cdot \delta(M)^{-1} \cdot \delta\left(M^{\prime}\right)^{-n} \\
& \sim(2 \pi i)^{\frac{n(n-1)}{2}} \cdot P_{\leqslant s\left(M^{\prime}\right)}(M) \cdot Q_{1}\left(M^{\prime}\right)^{-s\left(M^{\prime}\right)} \delta\left(M^{\prime}\right)^{-n}
\end{aligned}
$$

The polarizations define an isomorphism

$$
R\left(M \otimes M^{\prime}\right) \cong R\left(M \otimes M^{\prime}\right)^{\vee}(1-n+\kappa) \otimes \mathbb{Q}\left(\alpha_{0} \cdot \varepsilon_{\mathcal{K}}\right)
$$

and so, as before

$$
\begin{aligned}
c^{+} & \left(R\left(M \otimes M^{\prime}\right)(m)\right) \\
& \sim(2 \pi i)^{n(1-n+\kappa)+\frac{n(n-1)}{2}+n m} \mathcal{G}\left(\alpha_{0, f}^{-1} \cdot \varepsilon \mathcal{K}, f\right)^{n} \cdot P_{\leqslant s\left(M^{\prime}\right)}(M) \cdot Q_{1}\left(M^{\prime}\right)^{-s\left(M^{\prime}\right)} \delta\left(M^{\prime}\right)^{-n} \\
& \sim(2 \pi i)^{m n-\frac{n(n-1)}{2}} \mathcal{G}\left(\alpha_{0, f}^{-1} \cdot \varepsilon_{\mathcal{K}, f}\right)^{n} \cdot P_{\leqslant s\left(M^{\prime}\right)}(M) \cdot(2 \pi i)^{n \kappa} Q_{1}\left(M^{\prime}\right)^{-s\left(M^{\prime}\right)} \delta\left(M^{\prime}\right)^{-n} .
\end{aligned}
$$

\subsection{Holomorphic automorphic forms on unitary groups and automorphic critical intervals when $n^{\prime}=1$}

In [20] and its successor [21], expressions for the critical values of $L$-functions of unitary groups are derived from the determination of fields of rationality of Eisenstein series for the Siegel parabolic, on the one hand, and from the construction of holomorphic 
differential operators. The latter are used to specify the signature of the unitary similitude group whose associated Shimura variety realizes the critical values as the Petersson square norm of a (rational) holomorphic automorphic form, up to elementary factors. Unfortunately the definitions of the parameters are scattered in several places with confusing sign changes between successive appearances. For the reader's convenience, the definitions of all relevant parameters and the main results on the existence of differential operators are reproduced here.

Page numbers refer to [20]. The parameter $\left(\mu_{1}, \ldots, \mu_{n}\right)$ of $\Pi$ is denoted $\left(a_{1}, \ldots, a_{n}\right)$, starting on p. 104; we replace the $a$ 's with $\mu$ 's in what follows. There is also a central parameter c (denoted $c$ in [20]; we use boldface to distinguish the parameter from complex conjugation) and assumed (on p. 103) to be of the same parity as $\sum_{i=1}^{n} \mu_{i}$, and such that the motives realized on the degree $i$ cohomology of unitary group Shimura varieties are of weight $i-\mathbf{c}$ (see p. 104). The main results of [20] apply to self-dual $\Pi$ and with our conventions there it follows (though this is not adequately explained) that $\mathbf{c}=0$. Here we assume for simplicity that $\mathbf{c}=\sum_{i=1}^{n} \mu_{i}=C(\mu)$ in the notation of p. 105. This implies that the integers $\mathcal{P}(\mu)$ and $\mathcal{Q}(\mu)$ defined on p. 105 are given by

$$
\mathcal{P}(\mu)=-\mathbf{c} \text { and } \quad \mathcal{Q}(\mu)=0 .
$$

We fix a signature $(r, s)$, with $n=r+s$. For any integer $k$ one defines

$$
\begin{gathered}
\mu(k):=\left(\mu_{1}-k, \mu_{2}-k, \ldots, \mu_{n}-k ;-n k+\mathbf{c}\right), \\
\Lambda(\mu(k) ; r, s):=\left(\mu_{s+1}-k-s, \ldots, \mu_{n}-k-s ; \mu_{1}-k+r, \ldots, \mu_{s}-k+r ;-n k+\mathbf{c}\right) .
\end{gathered}
$$

(buried on p. 136 between (2.9.8) and (2.9.9), with $r$ and $s$ switched).

The motive $M(\Pi)$ attached abstractly to $\Pi$ has the Hodge types given by 4.2 . These cannot always be realized as cohomological motives, for several reasons. If $\Pi$ descends to an ( $L$-packet of) automorphic representations $\pi$ of a unitary group $U(V), V$ is a Hermitian space over $\mathcal{K}$ of signature $(r, s)$, then we obtain a motive with coefficients in $\mathcal{K}$, denoted $M^{\prime}(\pi, V ; r)$ on p. 118, in the cohomology of the corresponding Shimura variety, denoted $S h(V)$ in [20]. The $L$-packet contains the holomorphic type representation denoted $\pi_{r, s}$ above. When $\Pi$ is self-dual, as in [20], this is sufficient ${ }^{2}$, provided there is a unitary group $U(V)$ of signature $(n-1,1)$ to which $\Pi$ descends. When $n$ is even there is a local obstruction and one may only be able to realize even exterior powers of the desired $M(\Pi)$, even in the self-dual case. We therefore make the following hypothesis:

Hypotheses 4.19. For every signature $(r, s)$ with $r+s=n$, there is a unitary group $U(V)$ of signature $(r, s)$ such that the cuspidal automorphic representation $\Pi$ of $G\left(\mathbb{A}_{\mathcal{K}}\right)$ descends to an $L$-packet of automorphic representations $\{\pi\}$ of $U(V)$. Moreover, the $L$-packet $\{\pi\}$ contains a member whose archimedean component is in the holomorphic discrete series.

${ }^{2}$ More precisely, to get (the Betti realization of) a legitimate motive $M^{\prime}(\pi, V ; r)$ we need to take the sum $M^{\prime}(\pi, V ; r) \oplus M^{\prime}(\pi,-V ; s)$, as on p. 118 , and this is necessary to define the archimedean Frobenius as an operator on automorphic forms; but for the de Rham structure it suffices to work with $M^{\prime}(\pi, V ; r)$. 
This hypothesis will be assumed implicitly in the remainder of this section. In the statements of the main theorems in $\S 6$ the hypothesis will be included explicitly; there will also be a discussion of substitutes.

When $\Pi$ is conjugate self-dual but not self-dual - more precisely, when the central character $\omega_{\Pi}$ of $\Pi$ is not trivial, in particular when the integer $C(\mu)$ defined above is not even - there may be an additional obstruction. We need to choose a Hecke character $\xi$ such that

$$
\tilde{\xi}:=\xi / \xi^{c}=\omega_{\Pi}^{-1}
$$

Such a choice is always possible, cf. [6, Proposition 1.2.4] (which cites the book of Harris and Taylor). This is because the Shimura variety is defined in terms of $G U(V)$ and not $U(V)$, and an automorphic representation $\pi$ of $G U(V)$ gives rise by base change to a pair of automorphic representations $(\Pi, \xi)$ of $\mathrm{GL}_{n}\left(\mathbb{A}_{\mathcal{K}}\right) \times \mathrm{GL}_{1}\left(\mathbb{A}_{\mathcal{K}}\right)$. The parameter $\mathbf{c}$ is determined by the archimedean component $\xi_{\infty}$ of $\xi$ :

$$
\xi_{\infty}(t)=t^{-\mathbf{c}}
$$

The Hodge type of the motive realized in the cohomology of $\operatorname{Sh}\left(V_{1}\right)$, with $(r, s)=(n-1,1)$ (see [20, Example 2.2.5]) - call it $M^{\prime}\left(\pi, V_{1} ; n-1\right)$ (and don't confuse this $M^{\prime}$ with the $M^{\prime}$ for $\left.\mathrm{GL}_{n-1}\right)$ - is called the principal Hodge type in $[20, \S 2.2 .9]$; it is given by

$$
\left(p_{i}(\mu), q_{i}(\mu)\right)=\left(p_{i}+\mathcal{P}(\mu), q_{i}+\mathcal{Q}(\mu)\right)=\left(p_{i}-\mathbf{c}, q_{i}\right),
$$

where $\left(p_{i}, q_{i}\right)$ are attached to $\Pi$ as in 4.2 . Observe that by $(4.3)$, the pairs $\left(p_{i}, q_{i}\right)$ also depend on $\mu$ (although this has been hidden in the notation). More generally, if $V_{s}$ has signature $(r, s)$, the motive $M^{\prime}\left(\pi_{r, s}, V_{s} ; r\right)$ attached to the (holomorphic) representation $\pi_{r, s}$ of $G U(V)$ that is nearly equivalent to $\pi$, in the sense that $\pi$ and $\pi_{r, s}$ have the same base change $(\Pi, \xi)$ to $\mathrm{GL}_{n}\left(\mathbb{A}_{\mathcal{K}}\right) \times \mathrm{GL}_{1}\left(\mathbb{A}_{\mathcal{K}}\right)$, is expected to bear the following relation to the desired $M(\Pi)$ :

$$
M^{\prime}\left(\pi_{r, s}, V_{s} ; r\right) \cong \wedge^{r}\left(M(\Pi)^{\vee}\right) \otimes M\left(\xi^{c}\right) .
$$

This is known to be true in most cases at the level of Galois representations (cf. [6, Proposition 4.3.8], as well as the more complete results of Shin and Scholze-Shin), but in the absence of the Tate conjecture one can't even give a precise definition to the rank one motive $M\left(\xi^{c}\right)$, much less prove a result such as $(4.22)$. The best we can do is to show how the expressions for special values obtained by analytic methods are products of expressions that resemble those that would be expected if there were a complete theory of motives. The choice of $\xi$ should have no bearing on the expression of the special value of the $L$-function of $\Pi$; we return to this point below ${ }^{3}$.

We can now restate [20, Corollary 3.3.8], which is the main result on the existence of holomorphic differential operators. In what follows, notation is as on p. 145. In particular, $\mathcal{E}_{m, \kappa}$ is a line bundle on the Shimura variety $\operatorname{Sh}(n, n)$ (attached to a quasi-split unitary similitude group of size $2 n$ and $E_{\Lambda, \Lambda^{\sharp}(\kappa)}$ is an automorphic vector bundle on the Shimura variety $\operatorname{Sh}(V,-V)$ (essentially $\operatorname{Sh}(V) \times \operatorname{Sh}(-V))$.

${ }^{3}$ Actually, the Tate conjecture is more than we need. It would be enough to show that an isomorphism at the level of Galois representations implies an isomorphism at the level of motives for absolute Hodge cycles. 
Lemma 4.23. Let $n^{\prime}=1$ and $\Pi^{\prime}, M^{\prime}=M\left(\Pi^{\prime}\right)$, $k$, and $\kappa$ be as in 4.3. Let $(r, s)=$ $\left(r\left(M^{\prime}\right), s\left(M^{\prime}\right)\right)$ and let $V$ be a Hermitian space of signature $(r, s)$. Let $m$ be a critical value of $R\left(M \otimes M^{\prime}\right)$ to the right of (the central point) $\frac{1}{2}(n-\kappa)$. There is a (non-trivial) differential operator

$$
\Delta(m, \kappa, \Lambda): \mathcal{E}_{m, \kappa} \rightarrow E_{\Lambda, \Lambda}{ }^{\sharp}(\kappa)
$$

where $\Lambda=\Lambda\left(\mu^{\vee}(k) ; r, s\right)$ on the Shimura variety $\operatorname{Sh}(V,-V)$.

Proof. Keeping in mind that in absence of the self-duality hypothesis for $\mu$ we have to replace $\mu$ by $\mu^{\vee}$ in [20, Corollary 3.3.8], we obtain such a differential operator for the pair $(r, s)$, provided

$$
\begin{aligned}
\frac{1}{2}(n-\kappa) & \leqslant m \leqslant \min \left(q_{s+1}\left(\mu^{\vee}\right)+k-\kappa-\mathcal{Q}\left(\mu^{\vee}\right), p_{s}\left(\mu^{\vee}\right)-k-\mathcal{P}\left(\mu^{\vee}\right)\right) \\
& =\min \left(q_{s+1}^{c}+k-\kappa, p_{s}^{c}-k\right),
\end{aligned}
$$

where the second equality is $(4.21)$ for $\mu^{\vee}$. In particular, we have $2 q_{s+1}^{c}+2 k \geqslant n+\kappa$ and $2 p_{s}^{c}-2 k \geqslant n-\kappa ;$ in other words

$$
2 k-\kappa \in\left[n-2 q_{s+1}^{c}, 2 p_{s}^{c}-n\right] .
$$

So (4.16) implies that (4.24) is true exactly when $s=s\left(M^{\prime}\right)=s\left(M^{\prime}, M\right)$.

We now summarize informally the constructions of $[20, \S 3]$ and the corresponding sections of [21]. We let $\alpha$ and $\chi$ be Hecke characters of $\mathbb{A} \mathcal{K}$ and $U(1)(\mathbb{A})$, respectively, with parameters $\kappa$ and $k$ as above. Let $\pi$ be an anti-holomorphic representation of $G U(V)(\mathbb{A})$ and identify its contragredient $\pi^{\vee}$ with an anti-holomorphic representation of $G U(-V)(\mathbb{A})$ as in [20] (this identification is spread out over several sections; the most important statements are Corollary 2.5.11.2, Lemma 2.8.8, and (2.8.9.5)). If Eis $\in$ $\Gamma\left(\operatorname{Sh}(n, n), \mathcal{E}_{m, \kappa}\right)$ - in practice a holomorphic Eisenstein series - and $f \in \pi, f^{\prime} \in \pi^{\vee}$, and $f_{\chi}=f \otimes \chi \circ$ det, $f_{\chi}^{\prime}=f^{\prime} \otimes \chi^{-1} \circ$ det, then the value of the standard $L$-function at $s=m$ can be identified, up to elementary and local terms, with the cup product (Serre duality) pairing

$$
\Delta(m, \kappa, \Lambda) \cup\left[f_{\chi} \otimes\left(f_{\chi}^{\prime} \otimes \alpha^{-1} \text { odet }\right)\right] \rightarrow \mathbb{C} .
$$

This pairing is well defined provided (in the notation of p. 147)

$$
f_{\chi} \otimes\left(f_{\chi}^{\prime} \otimes \alpha^{-1} \circ \operatorname{det}\right) \in \bar{H}^{2 r s}\left(\operatorname{Sh}(V,-V), \Omega_{S h}^{t o p} \otimes E_{\Lambda, \Lambda^{\sharp}(\kappa)}^{\vee}\right)
$$

where $\Omega_{S h}^{t o p}$ is the canonical bundle on $\operatorname{Sh}(V,-V)$ and $\bar{H}$ is the version of coherent cohomology used in [20]. Now there is an isomorphism of automorphic vector bundles:

$$
\Omega_{S h}^{t o p} \otimes E_{\Lambda, \Lambda \sharp(\kappa)}^{\vee} \cong E_{\mu(-k), \mu(k-\kappa)},
$$

with $\Lambda=\Lambda\left(\mu^{\vee}(k) ; r, s\right)$ as in Lemma 4.23. This isomorphism is stated in the third displayed formula on [20, p. 150] with $\mu$ in place of $\mu^{\vee}$ inside $\Lambda$, which is legitimate in that setting because of the self-duality hypothesis. Now (4.27) implies that (4.26) holds, since $\Pi_{\infty}$ has cohomology with respect to $E_{\mu}$.

Therefore, the above calculations apply and give us the following version of $[20$, Theorem 3.5.13] and [21, Theorem 4.3]. Recall that when $\beta$ is a Hecke character, we 
have defined $\check{\beta}=\left(\beta^{c}\right)^{-1}$ on [20, p. 82]. This should not be confused with $\beta^{\vee}=\beta^{-1}$. In what follows, we let $\pi=\pi(\Pi, \xi)$ be an anti-holomorphic automorphic representation of $G U(V)(\mathbb{A})$, for some Hermitian space $V$ to be specified, whose base change equals $(\Pi, \xi)$. For any integer $j \in[0, n]$ define $P^{(j)}(\Pi, \xi)$ to be the period denoted $P^{(j)}(\pi, \star, \beta)$ in $[20,(2.8 .2)]$ : it is the Petersson inner product with itself of a rationally normalized holomorphic form on $G U(V)$ whose restriction to $U(V)$ is weakly equivalent to $\pi$ on a Hermitian space of signature $(n-j, j)$. We set

$$
P^{(j)}(\Pi):=(2 \pi)^{\mathbf{c}} P^{(j)}\left(\Pi^{\vee}, \xi^{-1}\right) \text {. }
$$

This can be shown to be independent of the choice of $\xi$ when appropriate $L$-functions have non-vanishing critical values (see 4.33 for an explanation). The twist by the contragredient is used for notational purposes only. We write

$$
G\left(\Pi^{\prime}\right):=(2 \pi i)^{k} p\left(\check{\chi}^{(2)} \cdot \check{\alpha}, 1\right) .
$$

The notation $\alpha_{f, 0}$ for the restriction of (the finite part of) a Hecke character to the idèles of $\mathbb{Q}$, and $\mathcal{G}$ for the Gauß sum, are as in $\S 3$.

Theorem 4.29. Let $m \geqslant \frac{1}{2}(n-\kappa)$ be a critical value of $L\left(s, R\left(M(\Pi) \otimes M\left(\Pi^{\prime}\right)\right)\right)$. Let $(r, s)=\left(r\left(M^{\prime}\right), s\left(M^{\prime}\right)\right)$ and let $V$ be a Hermitian space of dimension $n$ over $\mathcal{K}$ with signature $(r, s)$. Let $\xi$ be a Hecke character of $\mathcal{K}$ satisfying 4.20. Then,

$L\left(m, R\left(M(\Pi) \otimes M\left(\Pi^{\prime}\right)\right)\right) \sim_{\mathcal{K} E(\Pi) E\left(\Pi^{\prime}\right)}$

$$
(2 \pi i)^{m n-\frac{n(n-1)}{2}} \mathcal{G}\left(\varepsilon_{\mathcal{K}, f}\right)^{\left\lceil\frac{n}{2}\right\rceil} P^{(s)}\left(\Pi^{\vee}\right)\left[(2 \pi i)^{\kappa} \mathcal{G}\left(\alpha_{f, 0}^{-1}\right)\right]^{s} G\left(\Pi^{\prime}\right)^{n-2 s} .
$$

As mentioned above, this is a restatement of [20, Theorem 3.5.13] and [21, Theorem 4.3]. At the suggestion of the referee, we remind the reader that this result is proved by using the doubling method of Piatetski-Shapiro-Rallis and Garrett to identify the $L$-function with an integral of a degenerate Eisenstein series on a quasi-split unitary group to which the Shimura variety $\operatorname{Sh}(n, n)$ is attached, against antiholomorphic forms on the Shimura subvariety $\operatorname{Sh}(V,-V)$. The statement concerns critical values to the right of the center of symmetry; these correspond precisely to values of the Eisenstein series that are nearly holomorphic in Shimura's sense, and also to the differential operators described in 4.23 . The critical values can thus be analyzed in terms of cup products in coherent cohomology of $\operatorname{Sh}(V,-V)$. Note that the motivic interpretation of these critical values depends on an interpretation involving coherent cohomology, whereas the critical values of the Rankin-Selberg $L$-function of $\mathrm{GL}(n) \times \mathrm{GL}(n-1)$ are interpreted as cup products in topological cohomology.

Theorem 4.29 has the following equivalent reformulation. To this end, we recall that

$$
R\left(M(\Pi) \otimes M\left(\Pi^{\prime}\right)\right) \equiv R\left(M\left(\Pi^{c}\right) \otimes M\left(\Pi^{\prime, c}\right)\right)=R\left(M\left(\Pi^{\vee}\right) \otimes M\left(\Pi^{\prime, c}\right)\right) .
$$

Hence, by replacing $\mu$ by $\mu^{\vee}$ in (4.27), we may apply Theorem 4.29 to the pair $\left(M\left(\Pi^{\vee}\right), M\left(\Pi^{\prime, c}\right)\right)$, but still obtain an expression for our original $L$-value $L(m, R(M(\Pi) \otimes$ $\left.\left.M\left(\Pi^{\prime}\right)\right)\right)$. Let us write

$$
\Pi^{\prime, c}=B C(\chi)^{c} \alpha^{c}=B C\left(\chi^{\prime}\right) \cdot \alpha^{\prime}, \quad M^{\prime, c}=M\left(\Pi^{\prime, c}\right) .
$$


Then, a simple calculation shows that $k^{\prime}=-k+\kappa$ and $\kappa^{\prime}=\kappa$, where $k^{\prime}$ is attached to $\chi^{\prime}$ (respectively $\kappa^{\prime}$ to $\alpha^{\prime}$ ) as $k$ has been attached to $\chi$ (respectively $\kappa$ to $\alpha$ ). Hence, replacing $M^{\prime}$ by $M^{\prime}, c$ in (4.16), we obtain $2 k^{\prime}-\kappa^{\prime} \in\left[n-2 q_{s+1}^{c}(\Pi), 2 p_{s}^{c}(\Pi)-n\right]$ if and only if $s=s\left(M^{\prime, c}, M\right)$. Inserting the above values for $k^{\prime}$ and $\kappa^{\prime}$ and inverting the interval, we end up with

$$
2 k-\kappa \in\left[n-2 p_{s}^{c}(\Pi), 2 q_{s+1}^{c}(\Pi)-n\right]=\left[n-2 q_{r+1}(\Pi), 2 p_{r}(\Pi)-n\right],
$$

if and only if $r=r\left(M^{\prime, c}, M\right)$. Now, recall that

$$
2 k-\kappa \in\left[n-2 q_{s+1}(\Pi), 2 p_{s}(\Pi)-n\right]=\left[n-2 q_{s+1}^{c}\left(\Pi^{\vee}\right), 2 p_{s}^{c}\left(\Pi^{\vee}\right)-n\right]
$$

if and only if $s=s\left(M^{\prime}, M^{\vee}\right)$. Thus,

$$
\left(r\left(M^{\prime, c}, M^{\vee}\right), s\left(M^{\prime, c}, M^{\vee}\right)\right)=\left(s\left(M^{\prime}, M\right), r\left(M^{\prime}, M\right)\right) .
$$

We may hence reformulate Theorem 4.29 by

Theorem 4.31. Let $m \geqslant \frac{1}{2}(n-\kappa)$ be a critical value of $L\left(s, R\left(M(\Pi) \otimes M\left(\Pi^{\prime}\right)\right)\right)$. Let $(r, s)=\left(s\left(M^{\prime}\right), r\left(M^{\prime}\right)\right)$ and let $V$ be a Hermitian space of dimension $n$ over $\mathcal{K}$ with signature $(r, s)$. Let $\xi$ be a Hecke character of $\mathcal{K}$ satisfying 4.20. Then,

$L\left(m, R\left(M(\Pi) \otimes M\left(\Pi^{\prime}\right)\right)\right) \sim \mathcal{K} E(\Pi) E\left(\Pi^{\prime}\right)$

$$
\begin{aligned}
& (2 \pi i)^{m n-\frac{n(n-1)}{2}} \mathcal{G}(\varepsilon \mathcal{K}, f)^{\left\lceil\frac{n}{2}\right\rceil} P^{\left(r\left(M^{\prime}\right)\right)}(\Pi)\left[(2 \pi i)^{\kappa} \mathcal{G}\left(\alpha_{f, 0}^{-1}\right)\right]^{r\left(M^{\prime}\right)} \\
& \times G\left(\Pi^{\prime, c}\right)^{n-2 r\left(M^{\prime}\right)}
\end{aligned}
$$

\subsection{A comparison to Deligne's periods}

Now let us compare this to the critical value predicted by Deligne's conjecture, namely (4.18):

$$
(2 \pi i)^{m n-\frac{n(n-1)}{2}} \mathcal{G}\left(\alpha_{0, f}^{-1} \cdot \varepsilon \mathcal{K}, f\right)^{n} \cdot P_{\leqslant s\left(M^{\prime}\right)}(M) \cdot(2 \pi i)^{n \kappa} Q_{1}\left(M^{\prime}\right)^{-s\left(M^{\prime}\right)} \delta\left(M^{\prime}\right)^{-n} .
$$

Or rather let us make the following

Optimistic Comparison 4.32. Compare

$$
\mathcal{G}(\varepsilon \mathcal{K}, f)^{\left\lceil\frac{n}{2}\right\rceil} P^{\left(r\left(M^{\prime}\right)\right)}(\Pi)\left[(2 \pi i)^{\kappa} \mathcal{G}\left(\alpha_{0, f}^{-1}\right)\right]^{r\left(M^{\prime}\right)} G\left(\Pi^{\prime, c}\right)^{n-2 r\left(M^{\prime}\right)}
$$

to

$$
\mathcal{G}\left(\alpha_{0, f}^{-1} \cdot \varepsilon_{\mathcal{K}, f}\right)^{n} \cdot P_{\leqslant s\left(M^{\prime}\right)}(M) \cdot(2 \pi i)^{n \kappa} Q_{1}\left(M^{\prime}\right)^{-s\left(M^{\prime}\right)} \delta\left(M^{\prime}\right)^{-n} .
$$

Applying (4.22), and bearing in mind that the base change of $\pi$ is $(\Pi, \xi)$, we see that $i^{\mathbf{c}} P^{\left(r\left(M^{\prime}\right)\right)}(\Pi)$ is the correct normalization for the Petersson inner product of a rational class in the bottom stage of the Hodge filtration of $\wedge^{s\left(M^{\prime}\right)} M\left(\Pi^{\vee}\right) \otimes M\left(\left(\xi^{c}\right)^{-1}\right)$. (The presence of the power $i^{\mathbf{c}}$, which will disappear in our applications in any case, is explained in [20, Lemma 2.8.8].) On the other hand, $P_{\leqslant s\left(M^{\prime}\right)}(M)=Q_{\leqslant s\left(M^{\prime}\right)}(M) \cdot q(M)$ is the product with $q(M)$ of the Petersson norm of a rational class in the bottom stage of the Hodge filtration of $\wedge^{s\left(M^{\prime}\right)} M\left(\Pi^{\vee}\right)$, and plausible arguments can be made to justify identifying $(2 \pi i)^{\mathbf{c}} q(M)$ with the Petersson norm of $M\left(\left(\xi^{c}\right)^{-1}\right)$ (cf. [20, (3.7.9.3)-(3.7.9.5)], where however it is assumed that $\mathbf{c}=0)$. 
Remark 4.33. Note that the automorphic side (Aut) of the comparison involves a Petersson norm on the unitary similitude group, and therefore depends in principle on the auxiliary Hecke character $\xi$ defined in (4.20). The motivic side (Mot), on the other hand, is independent of this choice. Now Theorem 4.29 shows that replacing $\xi$ with $\xi^{\prime}$, also assumed to satisfy (4.20), gives another expression for the same critical value. In particular, if the critical value does not vanish, then we see that $P^{\left(r\left(M^{\prime}\right)\right)}\left(\Pi^{\vee}, \xi\right) \sim$ $P^{\left(r\left(M^{\prime}\right)\right)}\left(\Pi^{\vee}, \xi^{\prime}\right)$, where $\sim$ is taken over a simultaneous field of definition for $\Pi, \xi$, and $\xi^{\prime}$. The 'plausible arguments' mentioned above give a motivic interpretation for this identity.

It thus remains to justify an identification

$$
\begin{aligned}
\mathcal{G}\left(\alpha_{0, f}^{-1} \cdot \varepsilon_{\mathcal{K}, f}\right)^{n} \cdot & (2 \pi i)^{n \kappa} Q_{1}\left(M^{\prime}\right)^{-s\left(M^{\prime}\right)} \delta\left(M^{\prime}\right)^{-n} \\
& \sim \mathcal{G}(\varepsilon \mathcal{K}, f)^{\left\lceil\frac{n}{2}\right\rceil}(2 \pi i)^{\kappa r\left(M^{\prime}\right)} \mathcal{G}\left(\alpha_{0, f}^{-1}\right)^{r\left(M^{\prime}\right)} G\left(\Pi^{\prime, c}\right)^{n-2 r\left(M^{\prime}\right)} .
\end{aligned}
$$

We provide such a justification, at least up to Galois conjugation over $\mathcal{K}$, in the next section.

\subsection{The case of a Hecke character}

We now assume $n=1$ and $\Pi=\mathbf{1}$ is the trivial character, so $M=\mathbb{Q}(0)$. We have $s\left(M^{\prime}\right)=$ $s\left(M^{\prime}, \mathbb{Q}(0)\right)=1$ if $-2 k+\kappa \geqslant 1, s\left(M^{\prime}\right)=0$ if $-2 k+\kappa \leqslant 0$. Then, if $R\left(M^{\prime}\right)(m)$ is critical, (4.18) becomes

$$
c^{+}\left(R\left(M^{\prime}\right)(m)\right) \sim \begin{cases}(2 \pi i)^{m+\kappa} \mathcal{G}\left(\alpha_{0, f}^{-1} \cdot \varepsilon \mathcal{K}, f\right) \cdot Q_{1}\left(M^{\prime}\right)^{-1} \delta\left(M^{\prime}\right)^{-1}, & \text { if } 2 k-\kappa \leqslant-1 \\ (2 \pi i)^{m+\kappa} \mathcal{G}\left(\alpha_{0, f}^{-1} \cdot \varepsilon \mathcal{K}, f\right) \cdot \delta\left(M^{\prime}\right)^{-1} & \text { if } 2 k-\kappa \geqslant 0 .\end{cases}
$$

Since Deligne's conjecture is known for critical values of $L\left(s, R\left(M^{\prime}\right)\right)$, we thus have

$$
L\left(m, R\left(M^{\prime}\right)\right) \sim \begin{cases}(2 \pi i)^{m+\kappa} \mathcal{G}\left(\alpha_{0, f}^{-1} \cdot \varepsilon \mathcal{K}, f\right) \cdot Q_{1}\left(M^{\prime}\right)^{-1} \delta\left(M^{\prime}\right)^{-1}, & \text { if } 2 k-\kappa \leqslant-1 \\ (2 \pi i)^{m+\kappa} \mathcal{G}\left(\alpha_{0, f}^{-1} \cdot \varepsilon \mathcal{K}, f\right) \cdot \delta\left(M^{\prime}\right)^{-1} & \text { if } 2 k-\kappa \geqslant 0 .\end{cases}
$$

Here $\sim$ means $\sim_{\mathbb{Q}\left(M^{\prime}\right) ; \mathcal{K}}$ as in [20], because we are going to compare this with the result of Theorem 4.29 .

$$
L\left(m, R\left(M^{\prime}\right)\right) \sim(2 \pi i)^{m+r\left(M^{\prime}\right) \kappa} \mathcal{G}(\varepsilon \mathcal{K}, f) \mathcal{G}\left(\alpha_{0, f}^{-1}\right)^{r\left(M^{\prime}\right)} G\left(\Pi^{\prime, c}\right)^{1-2 r\left(M^{\prime}\right)} .
$$

Assume $s\left(M^{\prime}\right)=0$, so $2 k-\kappa \geqslant 0$. Then comparing (4.37) and (4.36) we find

$$
\mathcal{G}\left(\alpha_{0, f}^{-1} \cdot \varepsilon \mathcal{K}, f\right) \cdot \delta\left(M^{\prime}\right)^{-1} \sim \mathcal{G}(\varepsilon \mathcal{K}) \mathcal{G}\left(\alpha_{0, f}^{-1}\right) G\left(\Pi^{\prime, c}\right)^{-1} .
$$

Let us now get back to (4.34). Suppose for the moment $\alpha=1$, i.e., $\kappa=0$. So we compare $\mathcal{G}(\varepsilon \mathcal{K}, f)^{n} Q_{1}\left(M^{\prime}\right)^{-s\left(M^{\prime}\right)} \delta\left(M^{\prime}\right)^{-n}$ to $\mathcal{G}(\varepsilon \mathcal{K}, f)^{\left\lceil\frac{n}{2}\right\rceil} G\left(\Pi^{\prime, c}\right)^{n-2 r\left(M^{\prime}\right)}$. Inserting (4.38), we finally have to compare

$$
\mathcal{G}(\varepsilon \mathcal{K}, f)^{n} Q_{1}\left(M^{\prime}\right)^{-s\left(M^{\prime}\right)} \delta\left(M^{\prime}\right)^{-n}
$$

to

$$
\mathcal{G}(\varepsilon \mathcal{K}, f)^{\left\lceil\frac{n}{2}\right\rceil} \delta\left(M^{\prime}\right)^{n-2 r\left(M^{\prime}\right)}
$$


Now observe that [23, Lemma 1.2.7] and [23, (1.2.5)] imply that $Q_{1}\left(M^{\prime}\right) \sim \delta\left(M^{\prime}\right)^{-2}$. So we find that

$$
\mathcal{G}\left(\varepsilon_{\mathcal{K}, f}\right)^{n} Q_{1}\left(M^{\prime}\right)^{-s\left(M^{\prime}\right)} \delta\left(M^{\prime}\right)^{-n} \sim \mathcal{G}\left(\varepsilon_{\mathcal{K}, f}\right)^{n} \delta\left(M^{\prime}\right)^{2 s\left(M^{\prime}\right)-n} \sim \mathcal{G}\left(\varepsilon_{\mathcal{K}, f}\right)^{n} \delta\left(M^{\prime}\right)^{n-2 r\left(M^{\prime}\right)} .
$$

Hence, the two side of (4.34) coincide up to a power of the Gauß sum $\mathcal{G}(\varepsilon \mathcal{K}, f)$. We remark that the same conclusion holds if one uses the case $s\left(M^{\prime}\right)=1$ in order to obtain a relation for $G\left(\Pi^{\prime c}\right)$. Moreover, the case of non-trivial $\alpha$ is only slightly more complicated, and will not be used in the sequel.

\section{Rational Eisenstein classes of abelian type}

\subsection{Boundary cohomology}

Recall the abelian representation $\Pi^{\prime}$ from $\S 2.5$ and the space $S_{n-1}$ from $\S 3.1$. We denote by $\bar{S}_{n-1}$ the Borel-Serre compactification of $S_{n-1}$ and by $\partial \bar{S}_{n-1}$ its boundary; cf. [3, 41]. The face coming from the Borel subgroup $B^{\prime}$ of $G^{\prime}$ is denoted $\partial_{B^{\prime}} \bar{S}_{n-1}$. It is given by

$$
\partial_{B^{\prime}} \bar{S}_{n-1}=B^{\prime}(\mathcal{K}) \backslash G^{\prime}(\mathbb{A} \mathcal{K}) / K^{\prime} .
$$

Since the only automorphic representations of the torus $T^{\prime} \subset B^{\prime}$ are its Hecke characters $\chi$, it follows that

$$
H^{q}\left(\partial_{B^{\prime}} \bar{S}_{n-1}, \mathcal{E}_{\lambda}\right)=\bigoplus_{\chi} H^{q}\left(\mathfrak{g}^{\prime}, K^{\prime}, \operatorname{Ind}_{B^{\prime}\left(\mathbb{A}_{\mathcal{K}}\right)}^{G^{\prime}(\chi)}[\chi] \otimes E_{\lambda}\right)
$$

see, e.g., Harder [17]. Observe that induction is unitary here and that $\pi_{0}\left(G^{\prime}(\mathbb{C})\right)=$ $\pi_{0}\left(B^{\prime}(\mathbb{C})\right)=0$, which simplifies things. Recall the character $\tilde{\tau}=B C\left(\chi_{1}\right) \gamma \otimes \cdots \otimes$ $B C\left(\chi_{n-1}\right) \gamma$ from $\S 3.4$, and let $W\left(G^{\prime}\right)$ be the Weyl group of $G^{\prime}$. For $w \in W\left(G^{\prime}\right)$, we let $\tilde{\tau}^{w}$ be the representation

$$
\tilde{\tau}^{w}:=B C\left(\chi_{w^{-1}(1)}\right) \gamma \otimes \cdots \otimes B C\left(\chi_{w^{-1}(n-1)}\right) \gamma
$$

of $T^{\prime}\left(\mathbb{A}_{\mathcal{K}}\right)$, where $w$ is viewed as a permutation. In particular, we may write

$$
\begin{aligned}
H^{q}\left(\partial_{B^{\prime}} \bar{S}_{n-1}, \mathcal{E}_{\lambda}\right)= & \bigoplus_{w \in W\left(G^{\prime}\right)} H^{q}\left(\mathfrak{g}^{\prime}, K^{\prime}, \operatorname{Ind}_{B^{\prime}\left(\mathbb{A}_{\mathcal{K}}\right)}^{G^{\prime}\left(\mathbb{A}_{\mathcal{K}}\right)}\left[\tilde{\tau}^{w}\right] \otimes E_{\lambda}\right) \\
& \oplus \bigoplus_{\substack{\chi \neq \tilde{\tau}^{w} \\
\forall w \in W\left(G^{\prime}\right)}} H^{q}\left(\mathfrak{g}^{\prime}, K^{\prime}, \operatorname{Ind}_{B^{\prime}\left(\mathbb{A}_{\mathcal{K}}\right)}^{G^{\prime}\left(\mathbb{A}_{\mathcal{K}}\right)}[\chi] \otimes E_{\lambda}\right),
\end{aligned}
$$

and there is no weak $G^{\prime}\left(\mathbb{A}_{f}\right)$-intertwining between the two sums. Hence, for degree $q=$ $b_{n-1}$, we obtain

$$
H^{b_{n-1}}\left(\partial_{B^{\prime}} \bar{S}_{n-1}, \mathcal{E}_{\lambda}\right)=\bigoplus_{w \in W\left(G^{\prime}\right)} \operatorname{Ind}_{B^{\prime}\left(\mathbb{A}_{f}\right)}^{G^{\prime}\left(\mathbb{A}_{f}\right)}\left[\tilde{\tau}_{f}^{w}\right] \oplus \bigoplus_{\substack{\chi \neq \tilde{\tau}^{w} \\ \forall w \in W\left(G^{\prime}\right)}} H^{b_{n-1}}\left(\mathfrak{g}^{\prime}, K^{\prime}, \operatorname{Ind}_{B^{\prime}\left(\mathbb{A}_{\mathcal{K}}\right)}^{G^{\prime}\left(\mathbb{A}_{\mathcal{K}}\right)}[\chi] \otimes E_{\lambda}\right)
$$

and the summands in the first sum are all irreducible. 
Proposition 5.2. For all $\sigma \in \operatorname{Aut}(\mathbb{C})$, the following diagram of $G^{\prime}\left(\mathbb{A}_{f}\right)$-equivariant homomorphisms commutes:

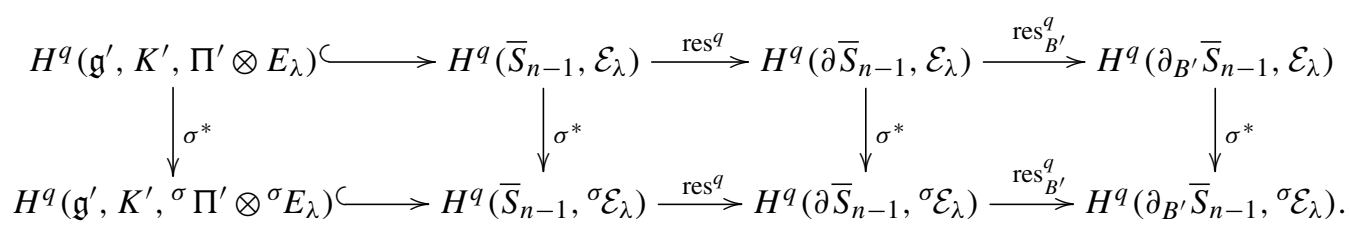

Furthermore, there are sections $s_{B^{\prime}}$ to the composition $r_{B^{\prime}}$ of the morphisms in the rows of the diagram,

$$
s_{B^{\prime}}: H^{b_{n-1}}\left(\partial_{B^{\prime}} \bar{S}_{n-1},{ }^{\sigma} \mathcal{E}_{\lambda}\right) \longrightarrow H^{b_{n-1}}\left(\mathfrak{g}^{\prime}, K^{\prime},{ }^{\sigma} \Pi^{\prime} \otimes{ }^{\sigma} E_{\lambda}\right) .
$$

Proof. We only prove the last assertion. Recall the archimedean vectors $\xi_{\Pi_{\infty}^{\prime}, j, \beta} \in$ $W\left(\Pi_{\infty}^{\prime}\right)$ from $\S 3.3$. Then, for every non-zero $\xi_{f} \in W\left(\Pi_{f}^{\prime}\right)$, we let

$$
E_{\Pi^{\prime}, \underline{j}, \beta}:=W^{-1}\left(\xi_{\Pi_{\infty}^{\prime}, \underline{j}, \beta} \otimes \xi_{f}\right) \in \Pi^{\prime}
$$

be the corresponding Eisenstein series. Hence,

$$
\sum_{\underline{j}, \beta}\left(X_{\underline{j}}^{*} \otimes E_{\Pi^{\prime}, \underline{j}, \beta} \otimes e_{\beta}^{\prime}\right) \in H^{b_{n-1}}\left(\mathfrak{g}^{\prime}, K^{\prime}, \Pi^{\prime} \otimes E_{\lambda}\right)
$$

is a non-zero cohomology class by the choice of $\xi_{\Pi_{\infty}^{\prime}, \underline{j}, \beta}$. Via the inclusion

$$
H^{b_{n-1}}\left(\mathfrak{g}^{\prime}, K^{\prime}, \Pi^{\prime} \otimes E_{\lambda}\right) \hookrightarrow H^{b_{n-1}}\left(\bar{S}_{n-1}, \mathcal{E}_{\lambda}\right),
$$

it is mapped onto

$$
\sum_{\underline{j}, \beta}\left(E_{\Pi^{\prime}, j, \beta} \otimes e_{\beta}^{\prime}\right) d x_{\underline{j}} \in H^{b_{n-1}}\left(\bar{S}_{n-1}, \mathcal{E}_{\lambda}\right),
$$

and finally, when restricting to the Borel stratum, we obtain

$$
\sum_{\underline{j}, \beta}\left(\left.E_{\Pi^{\prime}, \underline{j}, \beta}\right|_{B^{\prime}} \otimes e_{\beta}^{\prime}\right) d x_{\underline{j}} \in H^{b_{n-1}}\left(\partial_{B^{\prime}} \bar{S}_{n-1}, \mathcal{E}_{\lambda}\right) .
$$

Here, $\left.E_{\Pi^{\prime}, j, \beta}\right|_{B^{\prime}}$ is the constant term of the Eisenstein series $E_{\Pi^{\prime}, j, \beta}$ with respect to the Borel subgroup $B^{\prime}$; see [43, Satz 1.10]. According to [36, Proposition II.7], this constant term can be written as

$$
\left.E_{\Pi^{\prime}, j, \beta}\right|_{B^{\prime}}=\sum_{w \in W\left(G^{\prime}\right)} M(w, \tilde{\tau}) f_{\Pi^{\prime}, j, \beta},
$$

where $f_{\Pi^{\prime}, j, \beta}$ is a $K^{\prime}$-finite section in $\operatorname{Ind}_{B^{\prime}\left(\mathbb{A}_{\mathcal{K}}\right)}^{G^{\prime}\left(\mathbb{A}_{\mathcal{L}}\right]}[\tilde{c}$, used to define the Eisenstein series $E_{\Pi^{\prime}, \underline{j}, \beta}$, and $M(w, \tilde{\tau})$ is the corresponding intertwining operator,

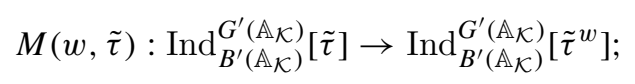


cf. [36, II.6]. As a consequence, the Eisenstein cohomology class, when restricting to the Borel stratum, yields the class

$$
\sum_{w \in W\left(G^{\prime}\right)} \sum_{\underline{j}, \beta}\left(M(w, \tilde{\tau}) f_{\Pi^{\prime}, \underline{j}, \beta} \otimes e_{\beta}^{\prime}\right) d x_{\underline{j}},
$$

which, via the isomorphism (5.1), lies in $\bigoplus_{w \in W\left(G^{\prime}\right)} \operatorname{Ind}_{B^{\prime}\left(\mathbb{A}_{f}\right)}^{G^{\prime}\left(\mathbb{A}_{f}\right)}\left[\tilde{\tau}_{f}^{w}\right]$. Here, we observe that there is no weak intertwining of the two sums in (5.1). This class is non-zero, since all Eisenstein series $E_{\Pi^{\prime}, \underline{j}, \beta}$ are holomorphic at their point of evaluation $s=0$; cf. [43, proof of Theorem 4.11]. Hence, since all representations $\left.\operatorname{Ind}_{B^{\prime}\left(\mathbb{A}_{f}\right)}^{G^{\prime}\left(\mathbb{A}_{f}\right)} \tilde{\tau}_{f}^{w}\right]$ are irreducible, the various summands of the image of $r_{B^{\prime}}$ are either trivial or the whole target space. The assertion follows now from the irreducibility of the source $\Pi_{f}^{\prime}=H^{b_{n-1}}\left(\mathfrak{g}^{\prime}, K^{\prime}, \Pi^{\prime} \otimes E_{\lambda}\right)$.

\subsection{A rationality result}

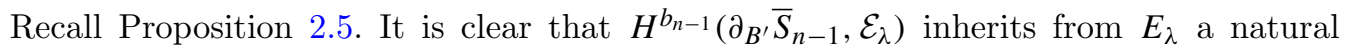
$\mathbb{Q}\left(E_{\lambda}\right)$-structure. By Proposition 2.4 and the temperedness of $\Pi_{\infty}^{\prime}$, there is the equality of number fields $\mathbb{Q}\left(E_{\lambda}\right)=\mathbb{Q}\left(\Pi_{\infty}^{\prime}\right)$. Hence, by Strong Multiplicity One for isobaric automorphic representations, $\mathbb{Q}\left(E_{\lambda}\right) \subseteq \mathbb{Q}\left(\Pi_{f}^{\prime}\right)$. Next, observe that, by Proposition 2.5, $\Pi_{f}^{\prime}=H^{b_{n-1}}\left(\mathfrak{g}^{\prime}, K^{\prime}, \Pi^{\prime} \otimes E_{\lambda}\right)$ carries a natural $\mathbb{Q}\left(\Pi_{f}^{\prime}\right)$-structure. We deduce the following corollary.

Corollary 5.3. The morphism $r_{B^{\prime}}$ is $\mathbb{Q}\left(\Pi_{f}^{\prime}\right)$-rational, i.e., it maps the natural $\mathbb{Q}\left(\Pi_{f}^{\prime}\right)$-structure of the cohomology $H^{b_{n-1}}\left(\mathfrak{g}^{\prime}, K^{\prime}, \Pi^{\prime} \otimes E_{\lambda}\right)$ to the natural $\mathbb{Q}\left(\Pi_{f}^{\prime}\right)$-structure of $H^{b_{n-1}}\left(\partial_{B^{\prime}} \bar{S}_{n-1}, \mathcal{E}_{\lambda}\right)$. The same holds true for the section $s_{B^{\prime}}$.

Proof. This is clear by the commutativity of the diagram in Proposition 5.2.

Remark 5.4. We would like to point out that for regular coefficients $E_{\lambda}$, Theorem 7.23 in Grobner and Raghuram [15], should provide an alternative approach to Corollary 5.3.

Recall from $\S 3.4$ that the rational structure on $\Pi_{f}^{\prime}$ is given by the natural rational structure on the induced representation. Let $\left.F \mapsto F\right|_{B^{\prime}}$ denote the constant term on functions (Eisenstein series) $F \in \Pi^{\prime}$, and let $\left.F\right|_{B^{\prime}}(\tilde{\tau})$ denote its $\tilde{\tau}$-component with respect to the action of the maximal torus in $B^{\prime}$. It follows from the proof of Proposition 5.2 that the following holds.

Lemma 5.5. Let $F=F_{\infty} \otimes F_{f} \in \Pi^{\prime}$ be a function with $F_{\infty}$ in the $K^{\prime}$-type contributing to the cohomology space $H^{b_{n-1}}\left(\mathfrak{g}^{\prime}, K^{\prime}, \Pi_{\infty}^{\prime} \otimes E_{\lambda}\right)$. Let $L \supseteq \mathbb{Q}\left(\Pi_{f}^{\prime}\right)$ be a field extension. Then $F$ defines an L-rational cohomology class $[F]$ if and only if $F_{f}$, viewed as an element of $\operatorname{Ind}_{B^{\prime}\left(\mathbb{A}_{f}\right)}^{G^{\prime}\left(\mathbb{A}_{f}\right)}\left[\tilde{\tau}_{f}\right]$, takes values in $L$, which is furthermore equivalent to $\left.F\right|_{B^{\prime}}(\tilde{\tau})$, viewed as an element of $\operatorname{Ind}_{B^{\prime}\left(\mathbb{A}_{f}\right)}^{G^{\prime}\left(\mathbb{A}_{f}\right)}\left[\tilde{\tau}_{f}\right]$, taking values in L. Moreover, for all $\sigma \in \operatorname{Aut}(\mathbb{C})$, $\sigma[F]={ }^{\sigma} F_{\infty} \otimes \sigma\left(F_{f}\right)$ (the notation having the obvious meaning). 


\subsection{Whittaker coefficients}

Let $f=\otimes_{w}^{\prime} f_{w}$ be a $K^{\prime}$-finite decomposable section in $\operatorname{Ind}_{B^{\prime}\left(\mathbb{A}_{\mathcal{K}}\right)}^{G^{\prime}}[\tilde{\tau}]$. We let $E(f)=E(f, 0)$ be the attached Eisenstein series, evaluated at 0, where it is always holomorphic. We will consider its global $\psi$-Whittaker functional ( $\psi$-Fourier coefficient):

$$
E_{\psi}(f)(g):=\int_{U^{\prime}(\mathcal{K}) \backslash U^{\prime}\left(\mathbb{A}_{\mathcal{K}}\right)} E(f)\left(u^{\prime} g\right) \psi^{-1}\left(u^{\prime}\right) d u^{\prime} \in W\left(\Pi^{\prime}\right) .
$$

To each 'function' $\varphi \in \tilde{\tau}$ (which is of course simply a constant), we can associate a $K^{\prime}$-finite decomposable section $f_{\varphi}=\otimes_{w}^{\prime} f_{\varphi, w} \in \operatorname{Ind}_{B^{\prime}\left(\mathbb{A}_{\mathcal{K}}\right)}^{G^{\prime}\left(\mathbb{A}_{\mathcal{\tau}}\right]}[$, as in $[42,6.3]$. Recall the choice of an element $w_{0} \in G^{\prime}(\mathcal{K})$, representing the longest element in the Weyl group of $G^{\prime} / \mathcal{K}$. Then we obtain the following result.

Proposition 5.6. The value at the identity $i d \in G^{\prime}\left(\mathbb{A}_{\mathcal{K}}\right)$ of the $\psi$-Whittaker functional $E_{\psi}\left(f_{\varphi}\right)$ equals

$$
E_{\psi}\left(f_{\varphi}\right)(\mathrm{id})=\prod_{w \in S\left(\Pi^{\prime}\right)} W_{w}\left(\mathrm{id}_{w}\right) \cdot \prod_{\substack{w \notin S\left(\Pi^{\prime}\right) \\ 1 \leqslant i<j \leqslant n-1}} L\left(1, B C\left(\chi_{i}\right)_{w} B C\left(\chi_{j}^{-1}\right)_{w}\right)^{-1},
$$

where

$$
W_{w}\left(\operatorname{id}_{w}\right)=\int_{U^{\prime}\left(\mathcal{K}_{w}\right)} f_{\varphi, w}\left(w_{0}^{-1} n_{w}^{\prime}\right) \psi_{w}^{-1}\left(n_{w}^{\prime}\right) d n_{w}^{\prime} .
$$

In particular, $E_{\psi}\left(f_{\varphi}\right)(\mathrm{id})$ has an Euler factorization.

Proof. This is [42, Theorem 7.1.2]. One simply observes that Shahidi's functional $\lambda_{w}^{0}$ (cf. [42, p. 122]) gives the complex number $f_{\varphi, w}\left(w_{0}^{-1} n_{w}^{\prime}\right)$, viewed as an element in $\tilde{\tau}$.

We recall the $\mathbb{Q}$-rational (and hence by our assumption also $\mathbb{Q}\left(E_{\lambda}\right)$-rational) basis vectors $X_{j}^{\prime *}$, respectively, the $\mathbb{Q}\left(E_{\lambda}\right)$-basis $\left\{e_{\beta}^{\prime}\right\}$ of $E_{\lambda}$ from $\S 3.3$. Moreover, we assume from now on that $\xi_{\Pi_{\infty}^{\prime}, j, \beta}$ was chosen in a way such that the generator $\left[\Pi_{\infty}^{\prime}\right]$ is $\mathbb{Q}\left(E_{\lambda}\right)$-rational in the sense of $\S \overline{2} .7$.

We now choose a section $f_{\varphi}$ such that $f_{\varphi, \infty}$ is one of the vectors $W^{-1}\left(\xi_{\Pi_{\infty}^{\prime}, \underline{j}, \beta}\right)$ appearing in the definition of $\left[\Pi_{\infty}^{\prime}\right]$ (or rather its transfer by $W^{-1}$ ), and that the non-archimedean part of $f_{\varphi}$ lies in the given $\mathbb{Q}\left(\Pi_{f}^{\prime}\right)$-structure of $\Pi_{f}^{\prime}$. Then, the evaluated Eisenstein series $E\left(f_{\varphi}\right)$ appears as a factor in the tensor product $\sum_{\underline{j}, \beta}\left(X_{\underline{j}}^{\prime *} \otimes E_{\Pi^{\prime}, \underline{j}, \beta} \otimes e_{\beta}^{\prime}\right)$, which defines a $\mathbb{Q}\left(\Pi_{f}^{\prime}\right)$-rational Eisenstein cohomology class.

Corollary 5.7. There is a constant $\Omega\left(\Pi_{\infty}^{\prime}\right) \in \mathbb{C}^{\times}$, depending only on $\Pi_{\infty}^{\prime}$ and on the choice of basis vectors $\xi_{\Pi_{\infty}^{\prime}, \underline{j}, \beta}$, such that

$$
p\left(\Pi^{\prime}\right) \sim_{\mathbb{Q}\left(\Pi_{f}^{\prime}\right)} \Omega\left(\Pi_{\infty}^{\prime}\right) \cdot \prod_{1 \leqslant i<j \leqslant n-1} L\left(1, B C\left(\chi_{i} \cdot \chi_{j}^{-1}\right)_{f}\right) .
$$

Proof. It follows from Corollary 5.3 and Lemma 5.5, and the definitions that the period $p\left(\Pi^{\prime}\right)$ can be represented by the ratio between the value at the identity of the $\tilde{\tau}$-component of the constant term and the value at the identity of the $\psi$-Whittaker 
functional $E_{\psi}\left(f_{\varphi}\right)$, if the latter does not vanish. We have chosen $f_{\varphi}$ in such a way that $E\left(f_{\varphi}\right)$ defines a $\mathbb{Q}\left(\Pi_{f}^{\prime}\right)$-rational class, which means that $p\left(\Pi^{\prime}\right)$ is the inverse of of $E_{\psi}\left(f_{\varphi}\right)(\mathrm{id})$, which by Proposition 5.6 equals

$$
\begin{aligned}
W_{\infty}\left(\operatorname{id}_{\infty}\right) & \cdot \prod_{\substack{w \in S\left(\Pi^{\prime}\right) \backslash\{\infty\} \\
1 \leqslant i<j \leqslant n-1}} W_{w}\left(\operatorname{id}_{w}\right) \cdot L\left(1, B C\left(\chi_{i}\right)_{w} B C\left(\chi_{j}^{-1}\right)_{w}\right) \\
\cdot & \prod_{1 \leqslant i<j \leqslant n-1} L\left(1, B C\left(\chi_{i}\right)_{f} B C\left(\chi_{j}^{-1}\right)_{f}\right)^{-1} .
\end{aligned}
$$

We take $\Omega\left(\Pi_{\infty}^{\prime}\right)=W_{\infty}\left(\mathrm{id}_{\infty}\right)^{-1}$. Since the local $L$-factors belong to $\mathbb{Q}\left(\Pi_{f}^{\prime}\right)$ and transform under the Galois group along with $\Pi^{\prime}$, we will finish the proof if we can show that $f_{\varphi, w}$ can be chosen in such a way that the integral defining $W_{w}\left(\mathrm{id}_{w}\right)$ is a rational constant. Now, as in Lemma 5.5, the $\mathbb{Q}\left(\Pi_{w}^{\prime}\right)$-rational structure on the principal series representation $\Pi_{w}^{\prime}$ is given by that on the boundary cohomology, and coincides with the one defined (adelically) in 2.6, namely the space of functions in the principal series whose restrictions to a fixed maximal compact subgroup $K_{w}^{\prime}$ take values in $\mathbb{Q}\left(\Pi_{w}^{\prime}\right)$. We take $U_{1} \subset U^{\prime}\left(\mathcal{K}_{w}\right)$ to be a subgroup of the kernel of $\psi_{w}$. Possibly shrinking $U_{1}$ further, we can assume that $f_{\varphi, w}\left(w_{0} n_{w}^{\prime}\right)$ is supported in $U_{1}$ as a function of $n_{w}^{\prime}$ and takes value 1 there. Then the integral is just a volume factor and belongs to $\mathbb{Q}$.

\section{Period relations - the main results}

In this section we always assume $\Pi$ and $\Pi^{\prime}$ to be obtained by base change from unitary groups of all signatures; in other words, that they both satisfy Hypothesis 4.19.

\subsection{Critical values of tensor products when one of the representations is of abelian type}

Let $\Pi^{\prime}$ be the abelian automorphic representation of 2.5 , attached to the $(n-1)$-tuple $\chi=\left(\chi_{1}, \ldots, \chi_{n-1}\right)$ and the auxiliary character $\gamma$; we write $\Pi^{\prime}=\Pi^{\prime}(\chi, \gamma)$. Recall that $\chi_{j, \infty}\left(e^{i \theta}\right)=e^{i k_{j} \theta}$ and $\gamma_{\infty}(z)=(z / \bar{z})^{t}$ for $k_{j} \in \mathbb{Z}$ and $t \in \frac{n}{2}+\mathbb{Z}$. If $n$ is odd, we henceforth assume $t=\frac{1}{2}$, to apply the formulas of [21].

Define $\gamma^{+}(x)$ as in Remark 2.6, i.e., $\gamma^{+}(x)=\gamma(x) \cdot\|x\|^{\frac{1}{2}}$ if $n$ is odd and $\gamma^{+}(x)=\gamma(x)$ if $n$ is even. Then, if $n$ is odd:

$$
\begin{aligned}
L\left(s, R\left(M(\Pi) \otimes M\left(\Pi^{\prime}\right)\right)\right) & =\prod_{j=1}^{n-1} L\left(s+\frac{3-2 n}{2}, \Pi \otimes B C\left(\chi_{j}\right) \cdot \gamma\right) \\
& =\prod_{j=1}^{n-1} L\left(s-\frac{n-1}{2}, M(\Pi) \otimes M\left(B C\left(\chi_{j}\right) \cdot \gamma^{+}\right)\right) .
\end{aligned}
$$

Moreover, if $n$ is odd, $\gamma_{\infty}^{+}(z)=z$ and thus, $\gamma^{+}$is of type $\eta_{\ell}$ with $\ell=-1$, in the notation of $[20,(2.9 .7)]$ and (especially) [21, Theorem 4.3], see p. 177. If $n$ is even, then $\gamma_{\infty}^{+}(z)=1$, so $\gamma^{+}$is of type $\eta_{\ell}$ with $\ell=0$. In what follows, we let $\alpha=\gamma^{+}$and so the restriction $\alpha_{0}=\varepsilon_{\mathcal{K}}^{n}$ for all $n$. Thus, with this choice of $\alpha$, the term $(2 \pi i)^{\kappa r\left(M^{\prime}\right)} \mathcal{G}\left(\alpha_{0, f}^{-1}\right)^{r\left(M^{\prime}\right)}$ 
in Theorem 4.31 is just $(2 \pi i)^{\kappa r\left(M^{\prime}\right)} \cdot \mathcal{G}\left(\varepsilon_{\mathcal{K}, f}^{n}\right)^{r\left(M^{\prime}\right)}$. Let $s_{0}=n-1+m$, where $m$ is as in 4.7 above. We apply Theorem 4.31 to the critical values of $L\left(s, R\left(M(\Pi) \otimes M\left(\Pi^{\prime}\right)\right)=\right.$ $L\left(s, R\left(M\left(\Pi^{\vee}\right) \otimes M\left(\Pi^{\prime c}\right)\right)\right.$. The results are as follows. If $n$ is even, $\alpha=\gamma^{+}$is the trivial character and we have

$$
\begin{aligned}
& L\left(n-1+m, R\left(M(\Pi) \otimes M\left(\Pi^{\prime}\right)\right)\right)=\prod_{j=1}^{n-1} L\left(\frac{n}{2}+m, M\left(\Pi^{\vee}\right) \otimes M\left(B C\left(\chi_{j}\right)^{c}\right)\right) \\
& \sim_{\mathcal{K} E(\Pi) E\left(\Pi^{\prime}\right)} \prod_{j=1}^{n-1}(2 \pi i)^{\left(\frac{n}{2}+m\right) n-\frac{n(n-1)}{2}} \cdot \mathcal{G}(\varepsilon \mathcal{K}, f)^{\frac{n}{2}} \cdot P^{\left(r\left(M_{j}^{+}\right)\right)}(\Pi) G\left(\Pi_{j}^{+, c}\right)^{n-2 r\left(M_{j}^{+}\right)} \\
& \sim_{\mathcal{K} E(\Pi) E\left(\Pi^{\prime}\right)}(2 \pi i)^{(n-1)(m n)+\frac{n(n-1)}{2}} \mathcal{G}(\varepsilon \mathcal{K}, f)^{\frac{n(n-1)}{2}} \prod_{j=1}^{n-1} P^{\left(r\left(M_{j}^{+}\right)\right)}(\Pi) G\left(\Pi_{j}^{+, c}\right)^{n-2 r\left(M_{j}^{+}\right)}, \\
& \sim_{\mathcal{K} E(\Pi) E\left(\Pi^{\prime}\right)}(2 \pi i)^{(n-1)(m n)+\frac{n(n-1)}{2}} \mathcal{G}(\varepsilon \mathcal{K}, f)^{\frac{n(n-1)}{2}} \prod_{j=1}^{n-1} P^{(j)}(\Pi) G\left(\Pi_{j}^{\prime, c}\right)^{n-2 j}
\end{aligned}
$$

with $\Pi_{j}^{+, c}=\left(B C\left(\chi_{j}\right) \gamma^{+}\right)^{c}=B C\left(\chi_{j}\right)^{-1}=\Pi_{j}^{c}$ and $M_{j}^{+}=M\left(\Pi_{j}^{+}\right)=M_{j}^{\prime}$. In order to obtain the last line, note that the character $\chi_{j}$ has infinity type $(z / \bar{z})^{k_{j}}$ which in the conventions of 4.2 means that the parameter is $-k_{j}$. Then the set $T\left(M, M_{j}^{\prime}\right)$ is the set of $t$ such that $2 k_{j} \geqslant n-2 p_{t}^{c}$. On the other hand, we are given that $2 k_{j} \in\left[n-2 q_{j+1}, 2 p_{j}-n\right]$, i.e., $j$ is the largest integer such that $2 k_{j} \geqslant n-2 q_{j+1}=n-2 p_{n-j}^{c}$. Thus, $s\left(M_{j}^{\prime}\right)=n-j$ by (4.16) and so $r\left(M_{j}^{+}\right)=r\left(M_{j}^{\prime}\right)=j$. If $n$ is odd, then $\alpha=\gamma^{+}$and we have

$$
\begin{aligned}
& L\left(n-1+m, R\left(M(\Pi) \otimes M\left(\Pi^{\prime}\right)\right)\right)=\prod_{j=1}^{n-1} L\left(\frac{n-1}{2}+m, M\left(\Pi^{\vee}\right) \otimes M\left(\left(B C\left(\chi_{j}\right) \gamma^{+}\right)^{c}\right)\right) \\
& \sim_{\mathcal{K} E(\Pi) E\left(\Pi^{\prime}\right)} \prod_{j=1}^{n-1}(2 \pi i)^{m n+r\left(M_{j}^{+}\right)} \mathcal{G}(\varepsilon \mathcal{K}, f)^{\left\lceil\frac{n}{2}\right\rceil+r\left(M_{j}^{+}\right)} P^{\left(r\left(M_{j}^{+}\right)\right)}(\Pi) G\left(\Pi_{j}^{+, c}\right)^{n-2 r\left(M_{j}^{+}\right)} \\
& \sim_{\mathcal{K} E(\Pi) E\left(\Pi^{\prime}\right)}(2 \pi i)^{(n-1)(m n)+\frac{n(n-1)}{2}} \mathcal{G}(\varepsilon \mathcal{K}, f)^{\left\lceil\frac{n}{2}\right\rceil(n-1)+\frac{n(n-1)}{2}} \prod_{j=1}^{n-1} P^{(j)}(\Pi) G\left(\Pi_{j}^{\prime, c}\right)^{n-2 j} .
\end{aligned}
$$

Here $\Pi_{j}^{+, c}=\left(B C\left(\chi_{j}\right) \gamma^{+}\right)^{c}=B C\left(\chi_{j}\right)^{-1}\left(\gamma^{+}\right)^{c}$. The contribution of $\gamma^{+}$disappears because the product of the periods of $\gamma^{+}$to the $n-2 j$ is just 1 . In particular, the expressions (6.2) and $(6.3)$ are identical (up to the power $\mathcal{G}\left(\varepsilon_{\mathcal{K}, f}\right)^{\frac{n(n-1)}{2}}$ of the Gauß-sum, which we may absorb into $\left.\mathcal{K} E(\Pi) E\left(\Pi^{\prime}\right)\right)$.

\subsection{Whittaker periods and Petersson norms}

In this section we combine the results of the previous sections to express the invariants $p(\Pi)$ in terms of the $P^{(s)}(\Pi)$. Notation is as above; in particular, $\Pi$ is cohomological and obtained by base change from unitary groups and $\Pi^{\prime}=\Pi^{\prime}(\chi, \gamma)$. 
Proposition 6.4. Suppose $\Pi$ satisfies Hypothesis 4.19. Let $s_{0}=n-1+m, m \geqslant 0$, be a critical value of $L\left(s, R\left(M(\Pi) \otimes M\left(\Pi^{\prime}\right)\right)\right)$ satisfying $L\left(n-1+m, R\left(M(\Pi) \otimes M\left(\Pi^{\prime}\right)\right)\right) \neq 0$. Then there are constants $p\left(m, \Pi_{\infty}, \Pi_{\infty}^{\prime}\right) \in \mathbb{C}^{\times}$and $\Omega\left(\Pi_{\infty}^{\prime}\right) \in \mathbb{C}^{\times}$such that

$$
\begin{gathered}
p\left(m, \Pi_{\infty}, \Pi_{\infty}^{\prime}\right) \Omega\left(\Pi_{\infty}^{\prime}\right) p(\Pi) \prod_{1 \leqslant i<j \leqslant n-1} L\left(1, B C\left(\chi_{i} \chi_{j}^{-1}\right)_{f}\right) \sim_{\mathcal{K} E(\Pi) E\left(\Pi^{\prime}\right)} L \\
(2 \pi i)^{(n-1)(m n)+\frac{n(n-1)}{2}} \mathcal{G}(\varepsilon \mathcal{K}, f)^{\left\lceil\frac{n}{2}\right\rceil(n-1)} \prod_{j=1}^{n-1} P^{(j)}(\Pi) G\left(\Pi^{\prime, c}\right)^{n-2 j}
\end{gathered}
$$

$A$ critical point $s_{0}=n-1+m, m \geqslant 0$, giving $L\left(n-1+m, R\left(M(\Pi) \otimes M\left(\Pi^{\prime}\right)\right)\right) \neq 0$ exists in particular, if the inequalities separating the archimedean parameters of $\Pi$ and $\Pi^{\prime}$ are strict:

$$
\mu_{1}>-\lambda_{n-1}>\mu_{2}>-\lambda_{n-2}>\cdots>-\lambda_{1}>\mu_{n}
$$

Proof. This is obtained by comparing the expressions of $3.9,5.7,6.2$, and 6.3 for the non-vanishing value of the $L$-function, and bearing in mind that, by 3.10 , the term $\mathcal{G}\left(\omega_{\Pi_{f, 0}^{\prime}}\right)$ in 3.9 is trivial. The last assertion follows from the description of 4.7 : It implies there is a critical value $s_{0}$ strictly to the right of the center of symmetry. Since $\Pi$ is cuspidal, it is a theorem of Shahidi and Jacquet-Shalika that the value $L\left(s_{0}, R(M(\Pi) \otimes\right.$ $\left.\left.M\left(\Pi^{\prime}\right)\right)\right) \neq 0$.

Now, let $M_{i j}^{\prime}:=M\left(B C\left(\chi_{i} \chi_{j}^{-1}\right)\right)$ with $i<j$. Then, the parameter of $M_{i j}^{\prime}$ is $2\left(k_{j}-k_{i}\right)<$ 0 . So $r\left(M_{i j}^{\prime}\right)=0$ and we get

$$
L\left(1, M_{i j}^{\prime}\right) \sim(2 \pi i) G\left(B C\left(\chi_{i} \chi_{j}^{-1}\right)^{c}\right)=(2 \pi i) G\left(\Pi_{i}^{\prime c}\right) G\left(\Pi_{j}^{\prime c}\right)^{-1}
$$

Then

$$
\prod_{1 \leqslant i<j \leqslant n-1} L\left(1, M_{i j}^{\prime}\right) \sim(2 \pi i)^{\frac{(n-1)(n-2)}{2}} \prod_{j=1}^{n-1} G\left(\Pi_{j}^{\prime c}\right)^{n-2 j} .
$$

Putting this together we find that, assuming the hypotheses of the preceding proposition are verified, we have

$$
\begin{gathered}
p\left(m, \Pi_{\infty}, \Pi_{\infty}^{\prime}\right) \Omega\left(\Pi_{\infty}^{\prime}\right) p(\Pi)(2 \pi i)^{\frac{(n-1)(n-2)}{2}} \prod_{j=1}^{n-1} G\left(\Pi_{j}^{\prime c}\right)^{n-2 j} \sim_{\mathcal{K} E(\Pi) E\left(\Pi^{\prime}\right)} \\
(2 \pi i)^{(n-1)(m n)+\frac{n(n-1)}{2}} \mathcal{G}\left(\varepsilon_{\mathcal{K}, f}\right)^{\left\lceil\frac{n}{2}\right\rceil(n-1)} \prod_{j=1}^{n-1} P^{(j)}(\Pi) \prod_{j=1}^{n-1} G\left(\Pi_{j}^{\prime c}\right)^{n-2 j}
\end{gathered}
$$

or more simply

$$
p\left(m, \Pi_{\infty}, \Pi_{\infty}^{\prime}\right) \Omega\left(\Pi_{\infty}^{\prime}\right) p(\Pi) \sim_{\mathcal{K} E(\Pi) E\left(\Pi^{\prime}\right)}(2 \pi i)^{(n m+1)(n-1)} \prod_{j=1}^{n-1} P^{(j)}(\Pi)
$$

Now, we can state our first main theorem: 
Theorem 6.7. Let $\mu$ be the parameter of $\Pi$, and suppose $\mu_{i}-\mu_{i+1} \geqslant 2$ for all $i$, so that there is a parameter $\lambda$ for $G^{\prime}$ and an integer $m>0$ such that $s_{0}=n-1+m$ is a critical value of the tensor product $L$-function $L\left(s, R\left(M(\Pi) \otimes M\left(\Pi^{\prime}\right)\right)\right.$ ) (in the motivic normalization) for any $\Pi^{\prime}$ with parameter $\lambda$. Suppose $\Pi$ satisfies Hypothesis 4.19 . Then there is a non-zero constant $Z\left(\Pi_{\infty}\right)$ which depends only on the local representation $\Pi_{\infty}$ and such that

$$
p(\Pi) \sim_{\mathcal{K} E(\Pi)} Z\left(\Pi_{\infty}\right) \prod_{j=1}^{n-1} P^{(j)}(\Pi) .
$$

Proof. The hypothesis on $\mu$ implies that there exist $\Pi^{\prime}$ of abelian type such that the comparison of (6.6) is valid. By letting $\Pi^{\prime}$ vary among abelian type representations with parameter $\lambda$, we can remove the $E\left(\Pi^{\prime}\right)$ from the equivalence relation. Initially one obtains the relation

$$
p(\Pi) \sim_{\mathcal{K} E(\Pi)} Z\left(m, \Pi_{\infty}, \Pi_{\infty}^{\prime}\right) \prod_{j=1}^{n-1} P^{(j)}(\Pi)
$$

where $Z\left(m, \Pi_{\infty}, \Pi_{\infty}^{\prime}\right)=\left[\Omega\left(\Pi_{\infty}^{\prime}\right) p\left(m, \Pi_{\infty}, \Pi_{\infty}^{\prime}\right)\right]^{-1} \cdot(2 \pi i)^{(n m+1)(n-1)}$. But since the left-hand side of 6.8 is independent of $m$ and $\Pi_{\infty}^{\prime}$, so is the right-hand side.

Remark 6.9. Two substitutes are possible for Hypothesis 4.19 when it is not satisfied. As noted above, there is only an obstruction if $n$ is even. Under the regularity hypothesis on the parameter $\mu$, it can be shown that the standard $L$-function of $\Pi$ can be realized, up to an abelian twist, as the $L$-function of a holomorphic automorphic representation of $U(n+1-j, j)$ for any $j$; this was done in most cases using the theta correspondence in [22]. The Petersson norm of an arithmetically normalized form in this representation can then be used in place of the missing $P^{(j)}(\Pi)$.

Even in the absence of the regularity hypothesis, one can use quadratic base change, as in [47], to define versions of the missing $P^{(j)}(\Pi)$. These are only well-defined up to square roots of elements in the coefficient field, so are less precise than the ones defined motivically.

\subsection{General tensor products}

Combining the comparison in Theorem 6.7 with Theorem 1.3, we obtain the following general result, when $\Pi_{\infty}$ and $\Pi_{\infty}^{\prime}$ are both sufficiently regular.

Theorem 6.10. Let $\Pi=B C(\pi)$ and $\Pi^{\prime}=B C\left(\pi^{\prime}\right)$ be cuspidal automorphic representations of $G\left(\mathbb{A}_{\mathcal{K}}\right)$ and $G^{\prime}\left(\mathbb{A}_{\mathcal{K}}\right)$ which are cohomological with respect to $E_{\mu}$ and $E_{\lambda}$, respectively. Suppose

(1) $\mu_{i}-\mu_{i+1} \geqslant 2$ for all $i$ and $\lambda_{j}-\lambda_{j+1} \geqslant 2$ for all $j$.

(2) Both $\Pi$ and $\Pi^{\prime}$ satisfy Hypothesis 4.19. 
Then for every critical point $s_{0}=\frac{1}{2}+m$ of $L\left(s, \Pi \times \Pi^{\prime}\right)$ with $m \geqslant 0$,

$$
\begin{aligned}
L\left(\frac{1}{2}+m, \Pi_{f} \times \Pi_{f}^{\prime}\right) & \sim \mathcal{K} E(\Pi) E\left(\Pi^{\prime}\right) \\
& \quad p\left(m, \Pi_{\infty}, \Pi_{\infty}^{\prime}\right) Z\left(\Pi_{\infty}\right) Z\left(\Pi_{\infty}^{\prime}\right) \prod_{j=1}^{n-1} P^{(j)}(\Pi) \prod_{k=1}^{n-2} P^{(k)}\left(\Pi^{\prime}\right) .
\end{aligned}
$$

Equivalently, for every critical point $s_{0}=n-1+m$ of $L\left(s, R\left(M(\Pi) \otimes M\left(\Pi^{\prime}\right)\right)\right)$ with $m \geqslant 0$,

$$
\begin{aligned}
L\left(n-1+m, R\left(M(\Pi) \otimes M\left(\Pi^{\prime}\right)\right)\right) & \sim \mathcal{K} E(\Pi) E\left(\Pi^{\prime}\right) \\
& p\left(m, \Pi_{\infty}, \Pi_{\infty}^{\prime}\right) Z\left(\Pi_{\infty}\right) Z\left(\Pi_{\infty}^{\prime}\right) \prod_{j=1}^{n-1} P^{(j)}(\Pi) \prod_{k=1}^{n-2} P^{(k)}\left(\Pi^{\prime}\right) .
\end{aligned}
$$

\subsection{The archimedean constant}

The constants $\Omega\left(\Pi_{\infty}^{\prime}\right), p\left(m, \Pi_{\infty}, \Pi_{\infty}^{\prime}\right)$, and $p(\Pi)$ all depend on choices of test vectors in the induced and Whittaker models of $\Pi_{\infty}$ and $\Pi_{\infty}^{\prime}$. We have decided to choose vectors rational over $\mathcal{K}$, but we could also choose test vectors with the property that $W_{\infty}\left(\Pi^{\prime}\right)=1$. Since this choice depends only on $\Pi_{\infty}^{\prime}$, it makes sense for cuspidal as well as Eisenstein representations. This provides a unique normalization of $p(\Pi)$ and thus a unique value for $p\left(m, \Pi_{\infty}, \Pi_{\infty}^{\prime}\right)$ (as does the choice of rational vectors).

In [33], Jie Lin proves a period relation for $\Pi$ and $\Pi^{\prime}$ obtained by automorphic induction from Hecke characters. Using this relation, she deduces the following refinement of Theorem 6.10.

Theorem 6.11 (Jie Lin). Under the hypotheses of Theorem 6.10, we have the following relations:

$$
L\left(\frac{1}{2}+m, \Pi_{f} \times \Pi_{f}^{\prime}\right) \sim_{\mathcal{K} E(\Pi) E\left(\Pi^{\prime}\right)}(2 \pi i)^{\left(m+\frac{1}{2}\right) n(n-1)} \prod_{j=1}^{n-1} P^{(j)}(\Pi) \prod_{k=1}^{n-2} P^{(k)}\left(\Pi^{\prime}\right)
$$

or equivalently,

$$
L\left(n-1+m, R\left(M(\Pi) \otimes M\left(\Pi^{\prime}\right)\right)\right) \sim_{\mathcal{K} E(\Pi) E\left(\Pi^{\prime}\right)}(2 \pi i)^{\left(m+\frac{1}{2}\right) n(n-1)} \prod_{j=1}^{n-1} P^{(j)}(\Pi) \prod_{k=1}^{n-2} P^{(k)}\left(\Pi^{\prime}\right),
$$

provided $m>0$.

If $m=0$ her result remains true under a certain global non-vanishing hypothesis. In view of formula (4.12), this is exactly what is predicted by Deligne's conjecture, assuming the validity of Optimistic Comparison 4.32.

\subsection{Whittaker periods and adjoint $L$-values}

The results of this section, in contrast to Theorem 6.7, are unconditional, but for the moment they only apply under slightly restrictive hypotheses. This is because they 
are based on Wei Zhang's version [51] of the Ichino-Ikeda-Neal Harris conjecture for automorphic forms on unitary groups, see [19], and for the moment neither the local nor global properties of the relative trace formula are known in sufficient generality to allow a complete comparison. The simplifying hypotheses in [51] are analogous to those used in earlier applications of the Arthur-Selberg trace formula, and the history of the latter gives us reason to be optimistic that the restrictions will soon be unnecessary.

For ease of reference, the simplifying hypotheses are listed separately.

6.5.1. Simplifying hypotheses. The unitary groups $H$ and $H^{\prime}$ are as in the earlier sections. We let $\pi$ and $\pi^{\prime}$ be unitary cuspidal automorphic representations of $H(\mathbb{A})$, respectively $H^{\prime}(\mathbb{A})$, which occur with multiplicity one in the discrete spectrum of $H$, respectively $H^{\prime}$. Say a prime $p$ is split if it splits in the quadratic extension $\mathcal{K}$. Since we want to use Wei Zhang's results, we have to assume

Hypotheses 6.12. - (a) There exists some split prime $p$ such that $\pi_{p}$ and $\pi_{p}^{\prime}$ are both supercuspidal.

- (b) Every prime $p<M$ is split, where $M$ is the 'algorithmically computable' constant that arises in [12] in the transfer to characteristic zero of the Jacquet-Rallis fundamental lemma (see below).

- (c) If $p$ is not split, then $\pi_{p}$ and $\pi_{p}^{\prime}$ are both unramified.

Remark 6.13. (a) Let $\Pi=B C(\pi)$ and $\Pi^{\prime}=B C\left(\pi^{\prime}\right)$ denote the base change representations of $\pi$ and $\pi^{\prime}$, to $G\left(\mathbb{A}_{\mathcal{K}}\right)$ and $G^{\prime}\left(\mathbb{A}_{\mathcal{K}}\right)$, respectively. Hypothesis (a) above implies that $\Pi$ and $\Pi^{\prime}$ are both cuspidal automorphic representations. Moreover, by Theorem 2.2, $\Pi$ and $\Pi^{\prime}$ are both of cohomological type. Hence, the Asai $L$-functions $L\left(s, \Pi, A s^{(-1)^{n-1}}\right)$ and $L\left(s, \Pi^{\prime}, A s^{(-1)^{n-2}}\right)$ have a pole at $s=1$, by [24] or [37, Corollary 2.5.9 and (2.5.12)]. As a consequence, Zhang's additional set of assumptions RH(I) (cf. [51], p. 544) is satisfied in our case. Moreover, Zhang's second set of assumptions RH(II) (cf. [51, pp. 544-545]) is fulfilled by the work of Beuzart-Plessis [2]. (We should, however, mention that the latter paper depends on the (expected) properties of local $L$-packets for unitary groups. See $[2, \S 18.1]$ for the precise expectations.)

(b) In [51] it is also assumed that $\pi$ and $\pi^{\prime}$ are locally tempered at all places. Due to Caraiani [4, Theorem 1.2], $\Pi=B C(\pi)$ and $\Pi^{\prime}=B C\left(\pi^{\prime}\right)$ are tempered at all places (under hypothesis (a) it was also proved in the book of Harris and Taylor). We expect that by [30] and forthcoming work of Kaletha et al., it should automatically follow that $\pi$ and $\pi^{\prime}$ are also tempered at all places, whence we did not assume this explicitly. However, the extremely careful reader may restrict himself/herself in what follows to the case where $\pi$ and $\pi^{\prime}$ are locally tempered at all primes $p$.

(c) Yun proves the Jacquet-Rallis fundamental lemma in [49] for local fields of characteristic $>n$. In her appendix to Yun's paper, Gordon derives the Jacquet-Rallis fundamental lemma for a local field $K$ of characteristic 0 , but at present her methods (from motivic integration) require her to assume that the residue characteristic of $K$ is greater than an unspecified positive constant $M$. This is the $M$ that appears in (b) above; it is effectively computable but no one has carried out the computation. For split 
primes the Jacquet-Rallis fundamental lemma is vacuous. The requirement that a prime $p$ be split in the results of [51] can be removed whenever the Jacquet-Rallis fundamental lemma is known in residue characteristic $p$.

Since $H(\mathbb{R})$ and $H^{\prime}(\mathbb{R})$ are both assumed compact we are in case (2) of [51], Theorem 1.2. We define

$$
\mathcal{L}\left(\pi, \pi^{\prime}\right):=\Delta_{n} \cdot \frac{L\left(\frac{1}{2}, \Pi \times \Pi^{\prime}\right)}{L(1, \pi, A d) L\left(1, \pi^{\prime}, A d\right)},
$$

where $\Delta_{n}$ is the $L$-function of the Gross motive:

$$
\Delta_{n}=\prod_{i=1}^{n} L\left(i, \varepsilon_{\mathcal{K}, f}^{i}\right) .
$$

For $\phi \in \pi$ and $\phi^{\prime} \in \pi^{\prime}$, we let

$$
\begin{aligned}
P\left(\phi, \phi^{\prime}\right) & :=\int_{H^{\prime}(\mathbb{Q}) \backslash H^{\prime}(\mathbb{A})} \phi\left(h^{\prime}\right) \phi^{\prime}\left(h^{\prime}\right) d h^{\prime} ; \\
\langle\phi, \phi\rangle & :=\int_{H(\mathbb{Q}) \backslash H(\mathbb{A})} \phi(h) \bar{\phi}(h) d h .
\end{aligned}
$$

We use Tamagawa measures for $d h$ and $d h^{\prime}$. Here is Zhang's theorem.

Theorem 6.14 [51]. Assume $\pi, \pi^{\prime}$, and $\mathcal{K}$ satisfy hypothesis 6.12. Then there is a non-zero constant $c\left(\pi_{\infty}, \pi_{\infty}^{\prime}\right)$, depending only on the archimedean components of $\pi$ and $\pi^{\prime}$, such that, for every factorizable $\phi \in \pi$ and $\phi^{\prime} \in \pi^{\prime}$,

$$
2^{-2} c\left(\pi_{\infty}, \pi_{\infty}^{\prime}\right) Z_{l o c}\left(\phi, \phi^{\prime}\right) \mathcal{L}\left(\pi, \pi^{\prime}\right)=\frac{\left|P\left(\phi, \phi^{\prime}\right)\right|^{2}}{\langle\phi, \phi\rangle\left\langle\phi^{\prime}, \phi^{\prime}\right\rangle} .
$$

Here $Z_{\text {loc }}=\prod_{v \in S} Z_{v}$ is a product of normalized local integrals of matrix coefficients; $Z_{v}$ is denoted

$$
\frac{\alpha_{v}^{\prime 4}\left(\Phi_{v}, \Phi_{v}\right)}{\left(\Phi_{v}, \Phi_{v}\right)_{v}}
$$

in [51], with $\Phi_{v}=\phi_{v} \otimes \phi_{v}^{\prime}$ (Zhang uses $\phi_{v}$ for this tensor product).

Remark 6.15. The theorem in [51] is stated under a more general version of hypothesis (c). With a bit more work we could derive the consequences below in this more general situation; however, hypothesis (c) is destined to disappear in the short term, so this seems unnecessary.

As in $[23,(4.1 .2)]$, we have

$$
\Delta_{n} \sim_{\mathcal{K}}(2 \pi i)^{\frac{n(n+1)}{2}} \cdot \mathcal{G}(\varepsilon \mathcal{K}, f)^{\left\lceil\frac{n+1}{2}\right\rceil} .
$$

In [23] $n$ is assumed even, but the same argument gives the above result. (Observe moreover that the field $\mathbb{Q}\left(\varepsilon_{\mathcal{K}}\right)$, which appears in [23] reduces to the field of rational 
numbers, as $\varepsilon_{\mathcal{K}}$ is a quadratic Hecke character.) Since $H$ and $H^{\prime}$ are both definite unitary groups, the archimedean local integral $Z_{\infty}$ is an algebraic number, rational over the field of definition of (the finite-dimensional representation) $\pi_{\infty} \otimes \pi_{\infty}^{\prime}$. The discussion leading to $[23, \S 4.1 .6]$ thus yields:

Corollary 6.17. Assume $\pi, \pi^{\prime}$, and $\mathcal{K}$ satisfy hypothesis 6.12 . Then there exists a non-zero constant $c_{\pi_{\infty}, \pi_{\infty}^{\prime}}$ such that

$$
\Lambda\left(\pi, \pi^{\prime}\right):=c_{\pi_{\infty}, \pi_{\infty}^{\prime}} \mathcal{G}(\varepsilon \mathcal{K}, f)^{\left\lceil\frac{n+1}{2}\right\rceil}(2 \pi i)^{\frac{n(n+1)}{2}} \cdot \frac{L\left(\frac{1}{2}, \Pi_{f} \times \Pi_{f}^{\prime}\right)}{L\left(1, \pi_{f}, A d\right) L\left(1, \pi_{f}^{\prime}, A d\right)} \in \overline{\mathbb{Q}} .
$$

- For all $\sigma \in \operatorname{Aut}(\mathbb{C})$,

$$
\sigma\left(\Lambda\left(\pi, \pi^{\prime}\right)\right)=\Lambda\left({ }^{\sigma} \pi,{ }^{\sigma} \pi^{\prime}\right) .
$$

The only difference with $[23,(4.1 .6 .2)]$ is the inclusion of the factor $c_{\pi_{\infty}, \pi_{\infty}^{\prime}}$ - since the Ichino-Ikeda-Neal Harris conjecture is only known up to a factor $c\left(\pi_{\infty}, \pi_{\infty}^{\prime}\right)$ - and of the power of the Gauß sum is not omitted.

Theorem 1.3 gives a different expression for the numerator of (6.18). Before we compare the two expressions, we need the following non-vanishing result, which is essentially due to Zhang.

Proposition 6.20. Let $\pi$ be an automorphic representation of $H(\mathbb{A})$ satisfying hypothesis 6.12. Then there exists an automorphic representation $\pi^{\prime}$ of $H^{\prime}(\mathbb{A})$ satisfying hypothesis 6.12 and $\phi \in \pi, \phi^{\prime} \in \pi^{\prime}$, such that $P\left(\phi, \phi^{\prime}\right) \neq 0$ and $L\left(\frac{1}{2}, B C(\pi) \times B C\left(\pi^{\prime}\right)\right) \neq 0$.

Proof. This is essentially [50, Lemma 2.15]. Let $p$ be a split place such that $\pi_{p}$ is supercuspidal. Since the center of $H$ is anisotropic, the central character of $\pi_{p}$ is unitary. Let $\mu_{p}$ be a supercuspidal representation of $H^{\prime}\left(\mathbb{Q}_{p}\right)$ with unitary central character. Since $H\left(\mathbb{Q}_{p}\right) \cong \mathrm{GL}_{n}\left(\mathbb{Q}_{p}\right)$ and $H^{\prime}\left(\mathbb{Q}_{p}\right) \cong \mathrm{GL}_{n-1}\left(\mathbb{Q}_{p}\right)$, it is known that $\operatorname{Hom}_{H^{\prime}\left(\mathbb{Q}_{p}\right)}\left(\left(\Pi_{p} \otimes\right.\right.$ $\left.\left.\mu_{p}\right), \mathbb{C}\right) \neq 0$. Since both $\pi_{p}$ and $\mu_{p}$ are tempered, it follows from a result of Sakellaridis and Venkatesh (quoted as [26, Theorem A.1]) that the local integral

$$
\int_{H^{\prime}\left(\mathbb{Q}_{p}\right)} f\left(h^{\prime}\right) f^{\prime}\left(h^{\prime}\right) d h^{\prime} \neq 0
$$

for some matrix coefficients $f, f^{\prime}$ of $\pi_{p}$ and $\mu_{p}$, respectively. It follows that (the dual of) $\mu_{p}$ is weakly contained in the restriction to $H^{\prime}\left(\mathbb{Q}_{p}\right)$ of $\pi_{p}$.

Now we assume $\mu_{p}$ is induced from an irreducible representation of $\mathrm{GL}_{n-1}\left(\mathbb{Z}_{p}\right) \cdot Z$, where $Z$ is the center of $H^{\prime}\left(\mathbb{Q}_{p}\right)$; by the theory of types, there are supercuspidal representations with this property. Choose an irreducible component $\mu_{\infty}$ of the restriction to $H^{\prime}(\mathbb{R})$ of (the finite-dimensional representation) $\pi_{\infty}$. Then we can apply [50, Lemma $2.15]$ to obtain an automorphic representation $\pi^{\prime}$ of $H^{\prime}$, whose archimedean component is $\mu_{\infty}$ and whose $p$-component is supercuspidal (a twist of $\mu_{p}$ ), for which there exist $\phi \in \pi$ and $\phi^{\prime} \in \pi^{\prime}$ such that $P\left(\phi, \phi^{\prime}\right) \neq 0$. This implies the non-vanishing of the $L$-value by the main result of [51], provided we know that $\pi^{\prime}$ is unramified at all non-split places. 
Lemma 6.21. Let $\pi$ be an irreducible representation of $H(\mathbb{A})$ when $n=1$; in other words, $\pi$ is a character of $U(1)(\mathbb{A})$. Then $L(s, \pi, A d)=L\left(s, \varepsilon_{\mathcal{K}}\right)$. In particular, $L\left(1, \pi_{f}, A d\right) \sim_{\mathcal{K}}$ $(2 \pi i) \mathcal{G}\left(\varepsilon_{\mathcal{K}, f}\right)$.

Proof. This is an obvious calculation: The Lie algebra of $H$ is one-dimensional and the adjoint representation of ${ }^{L} G$ is trivial on the Langlands dual group $\mathbb{C}^{\times}$and is given by the quadratic character $\varepsilon \mathcal{K}$.

Theorem 6.22. Let $\pi$ be an irreducible unitary cuspidal automorphic representation of $H(\mathbb{A})$ which is cohomological with respect to $E_{\mu}^{\mathrm{unt}}$. Assume $\pi$ satisfies hypothesis 6.12. Then there are a non-zero complex constant $a\left(\pi_{\infty}\right)$, depending only on $\pi_{\infty}$, and an integer $g(n) \in \mathbb{Z}$, such that

$$
p(B C(\pi)) \sim_{\mathcal{K} \mathbb{Q}(\pi)} a\left(\pi_{\infty}\right) \mathcal{G}(\varepsilon \mathcal{K}, f)^{g(n)} L\left(1, \pi_{f}, A d\right) .
$$

Proof. We prove this by induction on $n$. When $n=1$ the Whittaker period $p(B C(\pi))$ is rational and the claim follows from 6.21.

Suppose the theorem is known for $n-1$. Choose $\pi$ and let $\pi^{\prime}$ be an automorphic representation of $H^{\prime}(\mathbb{A})$ satisfying 6.20 relative to $\pi$. As $\pi$ and $\pi^{\prime}$ both satisfy hypothesis 6.12, $\Pi=B C(\pi)$ and $\Pi^{\prime}=B C\left(\pi^{\prime}\right)$ are cuspidal automorphic representations matching the conditions of Theorem 1.3. In particular, $p(B C(\pi))$ and $p\left(B C\left(\pi^{\prime}\right)\right)$ exist. By the very construction of $\pi^{\prime}$, Lemmas 2.1 and $4.7, s_{0}=\frac{1}{2}$ is critical for $L\left(s, B C(\pi) \times B C\left(\pi^{\prime}\right)\right)$, so $p\left(B C(\pi)_{\infty}, B C\left(\pi^{\prime}\right)_{\infty}\right)$ is well-defined. Now, let

$$
\Lambda_{W}\left(\pi, \pi^{\prime}\right):=c_{\pi_{\infty}, \pi_{\infty}^{\prime}} \mathcal{G}(\varepsilon \mathcal{K}, f)^{\left\lceil\frac{n+1}{2}\right\rceil}(2 \pi i)^{\frac{n(n+1)}{2}} \cdot \frac{p(B C(\pi)) p\left(B C\left(\pi^{\prime}\right)\right) p\left(B C(\pi)_{\infty}, B C\left(\pi^{\prime}\right)_{\infty}\right)}{L\left(1, \pi_{f}, A d\right) L\left(1, \pi_{f}^{\prime}, A d\right)} .
$$

We apply (6.18) and Theorem 1.3 to the pair $\left(\pi, \pi^{\prime}\right)$ and conclude that $\Lambda_{W}\left(\pi, \pi^{\prime}\right) \in$ $\overline{\mathbb{Q}}$. By [23, Proposition 2.6], ${ }^{\sigma} \Pi$ (respectively ${ }^{\sigma} \Pi^{\prime}$ ) descends to ${ }^{\sigma} \pi$ (respectively ${ }^{\sigma} \pi^{\prime}$ ), which are cuspidal automorphic representations satisfying hypothesis 6.12 . Hence, using Proposition 6.20, (6.19) and Theorem 1.3 again,

$$
\sigma\left(\Lambda_{W}\left(\pi, \pi^{\prime}\right)\right)=\Lambda_{W}\left({ }^{\sigma} \pi,{ }^{\sigma} \pi^{\prime}\right),
$$

for all $\sigma \in \operatorname{Aut}(\mathbb{C})$. But by induction, we can rewrite

$$
\begin{aligned}
& \Lambda_{W}\left(\pi, \pi^{\prime}\right) \sim \mathcal{K} \mathbb{Q}\left(\pi^{\prime}\right) \\
& \quad \frac{p(B C(\pi))}{L\left(1, \pi_{f}, A d\right)} \cdot\left[a\left(\pi_{\infty}^{\prime}\right) c_{\pi_{\infty}, \pi_{\infty}^{\prime}} \mathcal{G}(\varepsilon \mathcal{K}, f)^{\left\lceil\frac{n+1}{2}\right\rceil+g(n-1)}(2 \pi i)^{\frac{n(n+1)}{2}} \cdot p\left(B C(\pi)_{\infty}, B C\left(\pi^{\prime}\right)_{\infty}\right)\right] .
\end{aligned}
$$

It follows from $(6.23)$ that

$$
\begin{aligned}
& p(B C(\pi)) \sim_{\mathcal{K} \mathbb{Q}(\pi) \mathbb{Q}\left(\pi^{\prime}\right)} \\
& \quad L\left(1, \pi_{f}, A d\right) \cdot\left[a\left(\pi_{\infty}^{\prime}\right) c_{\pi_{\infty}, \pi_{\infty}^{\prime}} \mathcal{G}(\varepsilon \mathcal{K}, f)^{\left\lceil\frac{n+1}{2}\right\rceil+g(n-1)}(2 \pi i)^{\frac{n(n+1)}{2}} \cdot p\left(B C(\pi)_{\infty}, B C\left(\pi^{\prime}\right)_{\infty}\right)\right] .
\end{aligned}
$$

The term in brackets on the right-hand side depends only on $\pi_{\infty}$ (since $\pi_{\infty}^{\prime}$ is an irreducible component of the restriction to $H^{\prime}(\mathbb{R})$ of $\left.\pi_{\infty}\right)$ and the degree $n$, whereas the left-hand side depends only on $\pi$, so we conclude by induction. 
Remark 6.24. For a different approach to a similar (in fact more general) result, we refer to our forthcoming work with Lapid [14].

Corollary 6.25. Let $\Pi$ and $\Pi^{\prime}$ be cuspidal automorphic representations of $G\left(\mathbb{A}_{\mathcal{K}}\right)$ and $G^{\prime}\left(\mathbb{A}_{\mathcal{K}}\right)$ which are cohomological with respect to $E_{\mu}$ and $E_{\lambda}$, respectively. We assume $\Pi=$ $B C(\pi)$ and $\Pi^{\prime}=B C\left(\pi^{\prime}\right)$ where $\pi$ and $\pi^{\prime}$ are irreducible unitary cuspidal automorphic representations of the definite unitary groups $H(\mathbb{A})$ and $H^{\prime}(\mathbb{A})$, respectively. Suppose moreover that both $\pi$ and $\pi^{\prime}$ satisfy hypothesis 6.12 and that the coefficients $E_{\mu}$ and $E_{\lambda}$ satisfy the equivalent conditions of Lemma 2.1. Then for every critical point $s_{0}=\frac{1}{2}+m$ of $L\left(s, \Pi \times \Pi^{\prime}\right)$ with $m \geqslant 0$, there are a non-zero complex constant $a\left(m, \Pi_{\infty}, \Pi_{\infty}^{\prime}\right)$, depending only on the archimedean components of $\Pi$ and $\Pi^{\prime}$, and an integer $a(n)$, depending only on $n$, such that,

$$
L\left(\frac{1}{2}+m, \Pi_{f} \times \Pi_{f}^{\prime}\right) \sim_{\mathcal{K} \mathbb{Q}(\pi) \mathbb{Q}\left(\pi^{\prime}\right)} a\left(m, \Pi_{\infty}, \Pi_{\infty}^{\prime}\right) \mathcal{G}(\varepsilon \mathcal{K}, f)^{a(n)} L\left(1, \pi_{f}, A d\right) L\left(1, \pi_{f}^{\prime}, A d\right) .
$$

Equivalently, for every critical point $s_{0}=n-1+m$ of $L\left(s, R\left(M(\Pi) \otimes M\left(\Pi^{\prime}\right)\right)\right)$ with $m \geqslant$ 0 ,

$$
\begin{aligned}
& L\left(n-1+m, R\left(M(\Pi) \otimes M\left(\Pi_{f}^{\prime}\right)\right)\right) \\
& \quad \sim_{\mathcal{K} \mathbb{Q}(\pi) \mathbb{Q}\left(\pi^{\prime}\right)} a\left(m, \Pi_{\infty}, \Pi_{\infty}^{\prime}\right) \mathcal{G}\left(\varepsilon_{\mathcal{K}, f}\right)^{a(n)} L\left(1, \pi_{f}, A d\right) L\left(1, \pi_{f}^{\prime}, A d\right) .
\end{aligned}
$$

Proof. Indeed, it follows from the Theorems 6.22 and 1.3 and the fact that $\mathbb{Q}\left(\Pi_{f}\right) \subseteq \mathbb{Q}(\pi)$ (respectively $\left.\mathbb{Q}\left(\Pi_{f}^{\prime}\right) \subseteq \mathbb{Q}\left(\pi^{\prime}\right)\right)$ that we can define

$$
a\left(m, \Pi_{\infty}, \Pi_{\infty}^{\prime}\right):=a\left(\pi_{\infty}\right) a\left(\pi_{\infty}^{\prime}\right) p\left(m, \Pi_{\infty}, \Pi_{\infty}^{\prime}\right) \in \mathbb{C}^{\times}
$$

and $a(n):=g(n)+g(n-1) \in \mathbb{Z}$.

\subsection{Generalizations}

The methods of this paper apply to pairs $\Pi, \Pi^{\prime}$ of representations when the critical values of their $L$-functions can be related directly to cup products of the cohomology classes they define. This is only possible when the coefficients satisfy the inequalities of Lemma 2.1, a condition that is equivalent to relations on the Hodge types of the corresponding motives that are summarized as 4.6.

We have seen in 4.12 that the Deligne periods of tensor products of motives satisfying these hypotheses can be expressed, up to a certain power of $2 \pi i$, as products of terms denoted $P_{\leqslant s}(M)$ and $P_{\leqslant s^{\prime}}\left(M^{\prime}\right)$, each occuring to the first power (for $1 \leqslant s \leqslant n-1$ and $\left.1 \leqslant s^{\prime} \leqslant n-2\right)$. Deligne periods of tensor products are calculated more generally in [23, $\S 1.4]$. As explained in [23], the motivic periods that occur in this calculation belong to a tableau, and it follows without difficulty that in all cases these Deligne periods are products of certain integral powers of the same $P_{\leqslant s}(M)$ and $P_{\leqslant s^{\prime}}\left(M^{\prime}\right)$; the powers depend on the relative positions of the Hodge types of $M$ and $M^{\prime}$. In optimistic comparison 4.32 we argue that $P_{\leqslant s}(M)$ and $P_{\leqslant s^{\prime}}\left(M^{\prime}\right)$ can be identified with certain Petersson (square) norms of normalized holomorphic automorphic forms $P^{(i)}(\Pi)$ and $P^{(j)}\left(\Pi^{\prime}\right)$. Thus we would expect that the critical values of $L\left(s, \Pi \times \Pi^{\prime}\right)$ can always be expressed, up to algebraic factors, as powers of $2 \pi i$ multiplied by powers of these Petersson inner products. 
In fact, one can always find integers $N>n$ and Hecke characters $\chi_{i}, i=1, \ldots N-n$; $\chi_{j}^{\prime}, j=1, \ldots, N-n$, all obtained by base change from $U(1)$, such that the (tempered) Eisenstein representations

$$
\Sigma=\Pi \boxplus \chi_{1} \boxplus \chi_{2} \boxplus \cdots \boxplus \chi_{N-n} ; \quad \Sigma^{\prime}=\Pi^{\prime} \boxplus \chi_{1}^{\prime} \boxplus \chi_{2}^{\prime} \boxplus \cdots \boxplus \chi_{N-n}^{\prime}
$$

are cohomological and have coefficients satisfying the inequalities of Lemma 2.1. (In alluding to 'base change' we are ignoring the parity issue that we have already seen in 2.5; all the parameters of the Eisenstein representations have to be either integers or half-integers, depending on the parity of $N$. But this can always be arranged.) Suppose for the moment that the assertion of Theorem 3.9 was valid for the pair $\left(\Sigma, \Sigma^{\prime}\right)$. Then we would find that, up to algebraic factors, the critical values of $L\left(s, \Sigma \times \Sigma^{\prime}\right)$ were powers of $2 \pi i$ multiplied by

$$
\prod_{r=1}^{N-1} P^{(r)}(\Sigma) \cdot \prod_{r^{\prime}=1}^{N-2} P^{\left(r^{\prime}\right)}\left(\Sigma^{\prime}\right)
$$

On the other hand, the $L$-function admits a factorization

$$
L\left(s, \Sigma \times \Sigma^{\prime}\right)=L\left(s, \Pi \times \Pi^{\prime}\right) \cdot \prod_{i=1}^{N-n} L\left(s, \Pi^{\prime} \otimes \chi_{i}\right) \cdot \prod_{j=1}^{N-n} L\left(s, \Pi \otimes \chi_{j}^{\prime}\right) \cdot \prod_{i, j=1}^{N-n} L\left(s, \chi_{i} \cdot \chi_{j}\right) .
$$

Now we have expressions of the last three factors on the right-hand side in terms of $P^{(i)}(\Pi)$ and $P^{(j)}\left(\Pi^{\prime}\right)$ and $\mathrm{CM}$ periods of $\chi_{i}$ and $\chi_{j}^{\prime}$, and we can expect that $P^{(r)}(\Sigma)$ and $P^{\left(r^{\prime}\right)}\left(\Sigma^{\prime}\right)$ can also be expressed in terms of the periods of $\Pi, \Pi^{\prime}$, and the auxiliary Hecke characters. In this way one would obtain an expression of the critical values of interest, namely those of the remaining term $L\left(s, \Pi \times \Pi^{\prime}\right)$, as powers of $P^{(i)}(\Pi)$ and $P^{(j)}\left(\Pi^{\prime}\right)$, as expected.

The problem is that the integral representation used in Theorem 3.9 does not converge when the automorphic form on $\mathrm{GL}_{N}$ is an Eisenstein series. In [25], Ichino and Yamana obtain the Rankin-Selberg product for Eisenstein representations of $\mathrm{GL}_{N} \times \mathrm{GL}_{N-1}$ as a regularized period integral. It seems to be difficult to interpret this regularized integral as a regularized cup product in rational cohomology.

Alternatively, assuming both $\Sigma$ and $\Sigma^{\prime}$ descend to definite unitary groups, which must certainly be possible for appropriate choices of $\chi_{i}$ and $\chi_{j}^{\prime}$, we could apply the method described in $\S 6.5$ to obtain an expression for the central critical value $L\left(\frac{1}{2}, \Pi \times \Pi^{\prime}\right)$ analogous to that in Corollary 6.25 , provided the $\chi_{i}$ and $\chi_{j}^{\prime}$ could be chosen, with the given infinity types, so that the central critical values $L\left(\frac{1}{2}, \Pi^{\prime} \otimes \chi_{i}\right)$ and $L\left(\frac{1}{2}, \Pi \otimes \chi_{j}^{\prime}\right)$ were all non-zero. It would then be possible to deduce the expected expressions for more general central critical values from the expected generalization of recent results of Harder and Raghuram [18]. Unfortunately, proving the existence of such $\chi_{i}$ and $\chi_{j}^{\prime}$ seems to be an extremely difficult problem.

Yet another method would be to ignore the cup product altogether. The Rankin-Selberg integral

$$
\phi \otimes \phi^{\prime} \mapsto I_{m}\left(\phi, \phi^{\prime}\right):=\int_{G^{\prime}(\mathcal{K}) \backslash G^{\prime}\left(\mathbb{A}_{\mathcal{K}}\right)} \phi\left(g^{\prime}\right) \phi^{\prime}\left(g^{\prime}\right)\|\operatorname{det}(g)\|^{m} d g
$$


defines a $\left(\mathfrak{g}, K, G\left(\mathbb{A}_{f}\right)\right)$-invariant bilinear pairing on the space $\Pi_{(K)} \otimes \Pi_{\left(K^{\prime}\right)}^{\prime}$ of $K \times$ $K^{\prime}$-finite elements in $\Pi \otimes \Pi^{\prime}$, indexed by the integer $m$ (where this is defined by analytic continuation of the integral where necessary). On the other hand, its realization in cohomology determines a natural $\mathbb{Q}\left(\Pi_{f}\right) \mathbb{Q}\left(\Pi_{f}^{\prime}\right)$-rational structure on $\Pi_{f} \otimes \Pi_{f}^{\prime}$, and therefore $L\left(\Pi, \Pi^{\prime}\right):=\operatorname{Hom}_{\left(\mathfrak{g}, K, G\left(\mathbb{A}_{f}\right)\right)}\left(\Pi_{(K)} \otimes \Pi_{\left(K^{\prime}\right)}^{\prime}, \mathbb{C}\right)$ also has a natural $\mathbb{Q}\left(\Pi_{f}\right) \mathbb{Q}\left(\Pi_{f}^{\prime}\right)$-rational structure. Under the so-called automatic continuity hypothesis, any element of $L\left(\Pi, \Pi^{\prime}\right)$ extends continuously to the smooth Fréchet completions of moderate growth. In this case, it is known that $L\left(\Pi, \Pi^{\prime}\right)$ is of dimension 1 . Thus, there is a Rankin-Selberg period invariant $P\left(\Pi, \Pi^{\prime}\right) \in \mathbb{C}^{\times}$such that $P\left(\Pi, \Pi^{\prime}\right)^{-1} I_{m}$ is $\mathbb{Q}\left(\Pi_{f}\right) \mathbb{Q}\left(\Pi_{f}^{\prime}\right)$-rational; moreover, these invariants can be defined consistently for all Aut($(\mathbb{C})$-conjugates of $\Pi, \Pi^{\prime}$. Verification of Deligne's conjecture then comes down to identifying these $P\left(\Pi, \Pi^{\prime}\right)$, whether or not the pairing $I_{m}$ can be related to a cohomological cup product. If $\Pi^{\prime}$ is abelian automorphic, the $P\left(\Pi, \Pi^{\prime}\right)$ can be related as above to Petersson norms of holomorphic forms on unitary groups and periods of Hecke characters. It would seem that $P\left(\Pi, \Pi^{\prime}\right)$ factors in general as a product of a period invariant attached to $\Pi$ and one attached to $\Pi^{\prime}$, each independent of the other factor, but we see no way to prove this in general.

In a forthcoming joint paper with S. Yamana, we avoid these difficulties by applying a different combinatorial argument. We anticipate that our method will give a version of Theorem 6.10 without assuming the inequalities of Lemma 2.1.

Acknowledgements. We would like to thank Jie Lin for her endurance and great help in finding a vicious sign mistake in section 4, which troubled us while proof-reading the paper. We gratefully acknowledge the help of F. Shahidi and W. Zhang, for their answers to our questions about different steps in the proof of the main theorem. A. Raghuram helped us clear up an apparent contradiction between our results and Deligne's conjecture. We are especially grateful to Binyong Sun, for proving the non-vanishing theorem that makes our results unconditional and that will inevitably make the whole field of special values considerably more attractive.

\section{References}

1. A. Ash, Non-square-integrable cohomology classes of arithmetic groups, Duke J. Math. 47 (1980), 435-449.

2. R. Beuzart-Plessis, La conjecture locale de Gross-Prasad pour les représentations tempérées des groupes unitaires, Preprint, 2012, arXiv:1205.2987v2.

3. A. Borel and J.-P. Serre, Corners and arithmetic groups, Comm. Math. Helvet. 48 (1973), 436-491.

4. A. CARAiAni, Local-global compatibility and the action of monodromy on nearby cycles, Duke Math. J. 161 (2012), 2311-2413.

5. L. ClOzel, Motifs et Formes Automorphes: Applications du Principe de Fonctorialité, in Automorphic Forms, Shimura Varieties, and L-Functions, Vol. I, Ann Arbor, MI, 1988 (ed. L. Clozel And J. S. Milne), Perspectives in Mathematics, Volume 10, pp. 77-159 (Academic Press, Boston, MA, 1990).

6. L. Clozel, M. Harris and J.-P. Labesse, Construction of automorphic Galois representations, I, in The Stable Trace Formula, Shimura Varieties, and Arithmetic 
Applications. Volume I: Stabilization of the Trace Formula (ed. L. Clozel et al.), pp. 497-527 (International Press, Boston, 2011).

7. J. W. Cogdell and I. I. Piatetski-Shapiro, Remarks on Rankin-Selberg convolutions, in Contributions to Automorphic Forms, Geometry, and Number Theory (ed. H. HidA, D. RAmakrishnan AND F. Shahidi), pp. 255-278 (Johns Hopkins University Press, Baltimore, 2004).

8. P. Deligne, Valeurs de fonctions $L$ et périodes d'intégrales, with an appendix by N. Koblitz and A. Ogus, in Proc. Sympos. Pure Math., Volume XXXIII, part II, pp. 313-346 (American Mathematical Society, Providence, RI, 1979).

9. J. Franke, Harmonic analysis in weighted $L_{2}$-spaces, Ann. Sci. Éc. Norm. Supér. (4) 31 2 (1998), 181-279.

10. J. Franke And J. Schwermer, A decomposition of spaces of automorphic forms, and the Eisenstein cohomology of arithmetic groups, Math. Ann. 311 (1998), 765-790.

11. R. Goodman and N. Wallach, Symmetry, Representations, and Invariants, Graduate Texts in Mathematics, volume 255 (Springer, Dordrecht, 2009).

12. J. Gordon, Transfer to characteristic zero: Appendix to [49], Duke Math. J. 156 (2011), $220-227$.

13. H. Grobner, Residues of Eisenstein series and the automorphic cohomology of reductive groups, Compos. Math. 149 (2013), 1061-1090.

14. H. Grobner, M. HARris AND E. LAPID, Whittaker rational structures and special values of the Asai L-function, Contemp. Math. to appear.

15. H. Grobner and A. Raghuram, On some arithmetic properties of automorphic forms of GL $m$ over a division algebra, Int. J. Number Theory 10 (2014), 963-1013.

16. H. Grobner and A. Raghuram, On the arithmetic of Shalika models and the critical values of $L$-functions for $\mathrm{GL}_{2 n}$, with an appendix by Wee Teck Gan, Amer. J. Math. 136 (2014), 675-728.

17. G. HARDER, Some results on the Eisenstein cohomology of arithmetic subgroups of $\mathrm{GL}_{n}$, in Cohomology of Arithmetic Groups and Automorphic Forms (ed. J.-P. LABESSE AND J. Schwermer), Lecture Notes in Mathematics, Volume 1447, pp. 85-153 (Springer, 1990).

18. G. Harder and A. Raghuram, Eisenstein cohomology for $\mathrm{GL}_{N}$ and ratios of critical values of Rankin-Selberg $L$-functions - I, manuscript (2014).

19. R. N. HARris, The refined Gross-Prasad conjecture for unitary groups, Compos. Math. (to appear).

20. M. HARRIS, $L$-functions and periods of polarized regular motives, J. Reine Angew. Math. 483 (1997), 75-161.

21. M. HARris, A simple proof of rationality of Siegel-Weil Eisenstein series, in Eisenstein Series and Applications (ed. W. T. Gan, S. S. Kudla And Y. Tschinkel), Progress in Mathematics, Volume 258, pp. 149-186 (Birkhäuser Boston, Boston, 2008).

22. M. HARRIS, Cohomological automorphic forms on unitary groups, II: period relations and values of $L$-functions, in Harmonic Analysis, Group Representations, Automorphic Forms and Invariant Theory, Vol. 12 (ed. J.-S. LI et al.), Lecture Notes Series, pp. 89-150 (Institute of Mathematical Sciences, National University of Singapore, 2007).

23. M. HARRIs, L-functions and periods of adjoint motives, Algebra Number Theory 7 (2013), $117-155$.

24. M. Harris and J.-P. Labesse, Conditional base change for unitary groups, Asian J. Math. 8 (2004), 653-683. 
25. A. Ichino And S. Yamana, Periods of automorphic forms: the case of $\left(G L_{n+1} \times\right.$ $\mathrm{GL}_{n}, \mathrm{GL}_{n}$ ), Compos. Math. (in press).

26. A. ICHINO AND W. ZhANG, Spherical characters for a strongly tempered pair, appendix to $[50]$.

27. H. Jacquet, I. I. Piatetski-Shapiro and J. Shalika, Conducteur des représentations du groupe linéaire, Math. Ann. 256 (1981), 199-214.

28. H. Jacquet, I. I. Piatetski-Shapiro and J. Shalika, Rankin-Selberg convolutions, Amer. J. Math. 105 (1983), 367-464.

29. H. JACQUet AND J. Shalika, The Whittaker models of induced representations, Pacific J. Math. 109 (1983), 107-120.

30. T. Kaletha, A. Minguez, S. W. Shin and P.-J. White, Endoscopic classification of representations: inner forms of unitary groups, Preprint, 2014, arXiv:1409.3731.

31. J.-P. LABESSE, Changement de base CM et séries discrètes, in On the Stabilization of the Trace Formula, Vol. I (ed. L. Clozel, M. Harris, J.-P. Labesse and B.-C. NGô), pp. 429-470 (International Press, Boston, MA, 2011).

32. J.-S. Li And J. Schwermer, On the Eisenstein cohomology of arithmetic groups, Duke Math. J. 123 (2004), 141-169.

33. L. JIE, Period relations for automorphic induction and applications, I, manuscript, Comptes Rendus Math. 353 (2015), 95-100.

34. J. MAHnkopf, Cohomology of arithmetic groups, parabolic subgroups and the special values of automorphic L-Functions on GL(n), J. Inst. Math. Jussieu 4 (2005), 553-637.

35. J. Mahnkopf, Modular symbols and values of $L$-functions on $\mathrm{GL}_{3}$, J. Reine Angew. Math. 497 (1998), 91-112.

36. C. Megalin and J.-L. Waldspurger, Spectral Decomposition and Eisenstein Series (Cambridge University Press, 1995).

37. Сн. P. Mок, Endoscopic classification of representations of quasi-split unitary groups, Mem. Amer. Math. Soc. 235(1108) (in press).

38. A. Raghuram, Critical values of Rankin-Selberg $L$-functions for $\mathrm{GL}_{n} \times \mathrm{GL}_{n-1}$ and the symmetric cube $L$-functions for $\mathrm{GL}_{2}$. With an appendix by Chandrasheel Bhagwat, Preprint, 2014.

39. A. Raghuram, On the special values of certain Rankin-Selberg $L$-functions and applications to odd symmetric power $L$-functions of modular forms, Int. Math. Res. Not. IMRN 2010(2) (2009), 334-372.

40. A. Raghuram and F. Shahidi, On certain period relations for cusp forms on $\mathrm{GL}_{n}$, Int. Math. Res. Not. IMRN (2008), doi:10.1093/imrn/rnn077.

41. J. Rohlfs, Projective limits of locally symmetric spaces and cohomology, J. Reine Angew. Math. 479 (1996), 149-182.

42. F. Shahid, Eisenstein Series and Automorphic L-Functions, Colloquium Publications, Volume 58 (American Mathematical Society, 2010).

43. J. SCHWERMER, Kohomologie arithmetisch definierter Gruppen und Eisensteinreihen, Lecture Notes in Mathematics, Volume 988 (Springer-Verlag, 1983).

44. B. Sun, The nonvanishing hypothesis at infinity for Rankin-Selberg convolutions, Preprint, 2013, arXiv:1307.5357.

45. E. Urban, Formes automorphes cuspidales pour GL(2) sur un corps quadratique imaginaire. Valeurs spéciales de fonction $L$ et congruences, Compos. Math. 99 (1995), $283-324$.

46. J.-L. WALDSPURGER, Quelques propriétés arithmétiques de certaines formes automorphes sur GL(2), Compos. Math. 54 (1985), 121-171. 
47. H. Yoshida, On a conjecture of Shimura concerning periods of Hilbert modular forms, Amer. J. Math. 117 (1995), 1019-1038.

48. H. Yoshida, Motives and Siegel modular forms, Amer. J. Math. 123 (2001), 1171-1197.

49. Z. Yun, Duke Math. J. 156 (2011), 167-228.

50. W. ZHANG, Fourier transform and the global Gan-Gross-Prasad conjecture for unitary groups, Ann. of Math. 180 (2014), 971-1049.

51. W. Zhang, Automorphic period and the central value of Rankin-Selberg $L$-function, J. Amer. Math. Soc. 27 (2014), 541-612. 